\title{
Heating in Nanophotonic Traps for Cold Atoms
}

\author{
Daniel Hümmer, ${ }^{1,2}$ Philipp Schneeweiss, ${ }^{3}$ Arno Rauschenbeutel,,${ }^{3,4}$ and Oriol Romero-Isart ${ }^{1,2}$ \\ ${ }^{1}$ Institute for Quantum Optics and Quantum Information of the Austrian Academy of Sciences, \\ 6020 Innsbruck, Austria \\ ${ }^{2}$ Institute for Theoretical Physics, University of Innsbruck, 6020 Innsbruck, Austria \\ ${ }^{3}$ Atominstitut, TU Wien, 1020 Vienna, Austria \\ ${ }^{4}$ Department of Physics, Humboldt-Universität zu Berlin, 10099 Berlin, Germany
}

(Received 5 April 2019; revised manuscript received 26 September 2019; published 15 November 2019)

\begin{abstract}
Laser-cooled atoms that are trapped and optically interfaced with light in nanophotonic waveguides are a powerful platform for fundamental research in quantum optics as well as for applications in quantum communication and quantum-information processing. Ever since the first realization of such a hybrid quantum-nanophotonic system about a decade ago, heating rates of the atomic motion observed in various experimental settings have typically been exceeding those in comparable free-space optical microtraps by about 3 orders of magnitude. This excessive heating is a roadblock for the implementation of certain protocols and devices. Still, its origin has so far remained elusive and, at the typical atom-surface separations of less than an optical wavelength encountered in nanophotonic traps, numerous effects may potentially contribute to atom heating. Here, we theoretically describe the effect of mechanical vibrations of waveguides on guided light fields and provide a general theory of particle-phonon interaction in nanophotonic traps. We test our theory by applying it to the case of laser-cooled cesium atoms in nanofiber-based two-color optical traps. We find excellent quantitative agreement between the predicted heating rates and experimentally measured values. Our theory predicts that, in this setting, the dominant heating process stems from the optomechanical coupling of the optically trapped atoms to the continuum of thermally occupied flexural mechanical modes of the waveguide structure. Surprisingly, the effect of the high- $Q$ mechanical resonances which have previously been observed in this system can be neglected, even if they coincide with the trap frequencies. Beyond unraveling the long-standing riddle of excessive heating in nanofiber-based atom traps, we also study the dependence of the heating rates on the relevant system parameters and find a strong $R^{-5 / 2}$ scaling with the inverse waveguide radius. Our findings allow us to propose several strategies for minimizing the heating which also provide guidelines for the design of nextgeneration nanophotonic cold-atom systems. Finally, given that the predicted heating rate is proportional to the mass of the trapped particle, our findings are also highly relevant for optomechanics experiments with dielectric nanoparticles that are optically trapped close to nanophotonic waveguides.
\end{abstract}

DOI: 10.1103/PhysRevX.9.041034

Subject Areas: Atomic and Molecular Physics

\section{INTRODUCTION}

Small particles, such as laser-cooled atoms or dielectric nanospheres, are nowadays routinely trapped at submicron distances from solids. Structures currently investigated include photonic crystal waveguides [1-3], optical nanofibers [4-9], single carbon-nanotubes [10,11], dielectric membranes [12], and even macroscopic prisms [13,14]. The opportunities in research and application for systems combining atoms and solids are numerous, including the

Published by the American Physical Society under the terms of the Creative Commons Attribution 4.0 International license. Further distribution of this work must maintain attribution to the author(s) and the published article's title, journal citation, and DOI. search for novel fundamental forces [15-19], the implementation of quantum metrology and sensing using collective atomic state entanglement [20], and integrated quantum memories for photons guided in nanoscale waveguides [21-23]. A rich toolbox is already available for the cooling, trapping, positioning, and probing of atoms and nanoparticles. However, not all techniques commonly used in free-space traps for manipulating trapped particles are compatible with the presence of solid structures in their immediate proximity: Control laser beams, for instance, may be reflected or scattered in undesired ways. Moreover, additional effects such as van der Waals forces or coupling of the atoms or particles to thermal excitations in the solid have to be considered.

Full control at the quantum level over the internal as well as external degrees of freedom (d.o.f.) of individual atoms coupled to a nanophotonic structure was achieved only 
recently [24]. A key challenge in this context is the heating of the atomic motion observed in these systems $[25,26]$, which can reach rates of several hundred motional quanta per second-about 3 orders of magnitude larger than in comparable free-space optical traps. Large cooling rates realized, for example, by ultrastrong spin-motion coupling $[27,28]$, are required to overcome the heating and prepare atoms close to their motional ground state. In essence, the observed storage times of atoms in nanophotonic traps have fallen short of expectations, both for trapped cesium [2,5-7,9] and rubidium [8] atoms, ever since the first implementation of a nanofiber-based trap for laser-cooled atoms [4]. The origin of the strong heating and the corresponding low lifetimes has so far remained elusive. There is a range of conceivable causes, such as Raman scattering of the trapping light fields in the waveguide material [29], Brillouin scattering [30,31], or JohnsonNyquist noise [32]. However, estimates of their effect, provided as Supplemental Material [33], demonstrate that these mechanisms fail to explain heating rates observed in experiments. Additionally, tapered optical fibers, as used for realizing nanofiber-based cold-atom traps, exhibit thermally driven high- $Q$ torsional mechanical resonances which have been considered as a likely candidate for explaining the large heating in these systems [34]. In contrast, optical traps that are based on the evanescent field above a prism surface seem to feature small heating rates which are compatible, for instance, with BoseEinstein condensation of cesium atoms [35]. Indeed, even at room temperature, one does not expect thermally excited phonon modes of the macroscopic prism to contribute to the heating of the trapped atoms [36].

Here, we identify thermally populated flexural phononic modes of the nanoscopic waveguide as the dominant contributor to the large heating rates observed in nanofiber-based cold-atom traps. We give a concise description of the effect of mechanical modes on light guided in optical waveguides and provide a general theory of the resulting atom-phonon interaction in nanophotonic traps. Based on this formalism, we perform a case study for the cesium twocolor nanofiber-based trap described in Refs. [4,25,26]. Relying on independently measured system properties, we predict heating rates in excellent quantitative agreement with experimental observations. Surprisingly, the effect of the high- $Q$ torsional mechanical resonances that have previously been observed in this system [34] can be neglected, even if they coincide with the trap frequencies. We then use our model to numerically and analytically infer the scaling of the heating rates with system parameters such as the mechanical properties of the fiber, its temperature, or the trap frequencies. This systematic analysis allows us to outline strategies for minimizing the heating, thereby suggesting a solution to a long-standing problem of nanofiber-based cold-atom systems. While we formulate our theory in terms of atoms near nanofibers, it is indeed applicable to any kind of polarizable object trapped by conservative forces due to the light field surrounding a photonic structure. Building on the agreement obtained in the case study, our quantitative formalism might therefore be used for the faithful description of other nanophotonic cold-atom systems and, more generally, optomechanical systems with small particles, such as dielectric nanospheres [3,37-43], trapped in close vicinity to hot solid bodies.

This article is structured as follows: In Sec. II, we provide a general quantum theory describing atoms trapped in the optical near field of a vibrating photonic structure. In particular, we derive the general form of the atom-phonon interaction and discuss the resulting heating rates of the atomic motion. Section III: is dedicated to a case study of heating rates expected in a nanofiber-based two-color trap for laser-cooled atoms. In the Appendix A, we review the concept of photonic eigenmodes and summarize the modes of a nanofiber, while Appendix B recapitulates the resulting forces acting on trapped atoms. In Appendix C, we review quantized linear elastodynamics and summarize the phononic eigenmodes of a nanofiber. In Appendix D, we supply details on how to calculate the atom-phonon coupling constants based on the framework presented in Appendixes A-C. The parameters of the experimental setup considered in the case study are listed in Appendix E.

\section{ATOMS TRAPPED NEAR VIBRATING PHOTONIC STRUCTURES}

Micro- and nanophotonic traps rely on the optical near fields surrounding a photonic structure to spatially confine laser-cooled atoms in high vacuum. The optical fields are detuned from resonances of the atom such that they do not drive transitions between its internal (electronic) states. Confinement is achieved through gradients in the electric field that result in optical forces acting on the atom, analogous to free-space optical dipole traps [44]. In contrast to free-space setups, a dielectric photonic structure is used to pattern laser light in a way that creates local minima suitable for trapping atoms in the optical potential [45]. The light can either be guided by the structure such that atoms interact with the evanescent fields surrounding it $[4,5,46-52]$, or scattered by the structure $[1,2,53,54]$; see Appendix A. In either case, a fraction of the light is absorbed, which can lead to a bulk temperature of the dielectric of several hundred kelvins due to the weak thermal coupling to its environment [55]. In consequence, mechanical modes of the photonic structure are thermally excited. These mechanical modes (phonons) are in turn coupled to the external (motional) state of trapped atoms through the optical forces and other forces acting between the atoms and the structure.

An individual atom trapped in the optical near field surrounding a mechanically vibrating photonic structure suspended in high vacuum can be modeled by the Hamiltonian 


$$
\hat{H}=\hat{H}_{\mathrm{at}}+\hat{H}_{\mathrm{phn}}+\hat{H}_{\mathrm{at}-\mathrm{phn}} .
$$

The first term describes the dynamics of the trapped atom in the absence of phonons. Atoms are trapped at a distance of a few hundred nanometers from the surface of the structure because the near fields decay on a scale given by the optical wavelength. At such distances, corrections $\hat{V}_{\text {ad }}$ to the optical potential $\hat{V}_{\text {opt }}$ due to surface effects like dispersion forces become relevant [49,56]. Optical forces and dispersion forces are additive to first order [57]; hence, the total potential experienced by the atom is $\hat{V}_{0} \equiv \hat{V}_{\text {opt }}+\hat{V}_{\text {ad }}$. While the potential in general couples all atomic d.o.f. [24,28], we focus on scenarios without coupling of electronic and motional states and assume that the atom does not change its internal state. In this case $\hat{V}_{0}=V_{0}(\hat{\boldsymbol{r}})$; that is, the center of mass of the atom is subject to a potential $V_{0}$ which depends on the internal state of the atom (see Appendix B). Approximating the potential as harmonic for an atom close to its trapped motional ground state yields the atom Hamiltonian

$$
\hat{H}_{\mathrm{at}} \equiv \sum_{i} \hbar \omega_{i} \hat{a}_{i}^{\dagger} \hat{a}_{i}
$$

where $i$ labels the three orthogonal symmetry axes of the potential in harmonic approximation, $\omega_{i}$ are the trap frequencies, $\hbar$ is the reduced Planck constant, and $\hat{a}_{i}$ and $\hat{a}_{i}^{\dagger}$ are ladder operators for the harmonic motion of the trapped atom.

The second term $\hat{H}_{\text {phn }}$ in Eq. (1) describes the free evolution of the phonon field of the photonic structure. Vibrations at frequencies relevant to atom traps can be modeled by linear elasticity theory because the corresponding phonon wavelengths are sufficiently large not to resolve the microscopic structure of the solid. Linear elasticity theory describes the dynamics of elastic deformations of a continuous body around its equilibrium configuration [58-60]. The deformations are described by the displacement field $\boldsymbol{u}$, a real-valued vector field which indicates magnitude and direction of the displacement of each point of the body from equilibrium at a given time. A quantum formulation of linear elasticity theory can be obtained through canonical quantization based on phononic eigenmodes; see Appendix C. The eigenmodes can be labeled by a suitable multi-index $\gamma$ which may contain both discrete and continuous indices. In terms of ladder operators $\hat{b}_{\gamma}$ and $\hat{b}_{\gamma}^{\dagger}$ of the phonon field, the resulting phonon Hamiltonian is

$$
\hat{H}_{\mathrm{phn}} \equiv \sum_{\gamma} \hbar \omega_{\gamma} \hat{b}_{\gamma}^{\dagger} \hat{b}_{\gamma}
$$

where the sum symbolizes an integral in the case of the continuous index components.
The last term $\hat{H}_{\text {at-phn }}$ in the Hamiltonian Eq. (1) describes the coupling between the atomic motion and the phonon field. In order to obtain explicit expressions for the atomphonon coupling, it is necessary to know how the potential experienced by the atom is changed by vibrations. Here, we give an overview of how this dependence can be modeled, while further details as well as explicit expressions for the resulting coupling constants in the case of a nanofiberbased atom trap are provided in Appendix D. The coupling arises both because vibrations displace the photonic structure relative to the atom and because they change the electromagnetic properties of the structure in two ways [61]: First, vibrations deform the surface of the structure, as determined by the displacement field $\boldsymbol{u}$. Second, they locally change the refractive index and introduce birefringence (photoelastic effect), as determined by the strain tensor $\boldsymbol{S}$. The strain tensor describes deformations of the solid and has components $S^{i j} \equiv\left(\partial_{i} u^{j}+\partial_{j} u^{i}\right) / 2$, where $\partial_{i}$ indicates a spatial derivative. Both effects modify the photonic eigenmodes and hence the optical trapping fields. The optical fields and surface forces adapt to changes caused by vibrations on a timescale that is fast compared to the motion of the trapped atom. We can therefore treat the total potential as a functional $V[\boldsymbol{u}, \boldsymbol{S}](\boldsymbol{r})$ which, in the absence of vibrations, reduces to the potential $V[\mathbf{0}, \mathbf{0}](\boldsymbol{r}) \equiv$ $V_{0}(\boldsymbol{r})$ included in $\hat{H}_{\text {at }}$.

Thermal vibrations only weakly modify the atom trap. In consequence, it is justified to expand the potential to linear order around $\boldsymbol{u}=\mathbf{0}$ and $\boldsymbol{S}=\mathbf{0}$, and approximate $V[\boldsymbol{u}, \boldsymbol{S}] \simeq V_{0}+D V_{(\mathbf{0}, \mathbf{0})}[\boldsymbol{u}, \boldsymbol{S}]$. The first-order term is the functional derivative of $V\left[\boldsymbol{u}^{\prime}, \boldsymbol{S}^{\prime}\right]$ evaluated at $\left(\boldsymbol{u}^{\prime}, \boldsymbol{S}^{\prime}\right)=$ $(\mathbf{0 , 0})$ and in direction $(\boldsymbol{u}, \boldsymbol{S})$; see Ref. [62]. This term approximates phonon-induced variations of the potential and acts as the atom-phonon interaction Hamiltonian

$$
\hat{H}_{\mathrm{at}-\mathrm{phn}} \equiv D V_{(\mathbf{0}, \mathbf{0})}[\hat{\boldsymbol{u}}, \hat{\boldsymbol{S}}](\hat{\boldsymbol{r}}) .
$$

Truncating the expansion at linear order corresponds to assuming that the atom interacts only with single phonons at a time. Since the potential depends on both displacement and strain, there are two contributions to the interaction Hamiltonian, a displacement coupling (dp) due to the direct dependence of the potential on $\boldsymbol{u}$ and a strain coupling (st) due to the dependence on $S$ :

$$
\hat{H}_{\mathrm{at}-\mathrm{phn}}=\delta_{\boldsymbol{u}} V_{(\mathbf{0 , 0})}[\hat{\boldsymbol{u}}]+\delta_{S} V_{(\mathbf{0 , 0})}[\hat{\boldsymbol{S}}],
$$

Here, $\delta$ is the partial functional derivative [62]. The interaction Hamiltonian is linear in $\hat{\boldsymbol{u}}$ and $\hat{S}$ because the functional derivative is linear. By expanding displacement and strain in terms of phononic eigenmodes, the Hamiltonian can thus be expressed in terms of a positiondependent, complex-valued coupling function $g_{\gamma}(\boldsymbol{r})$ for each phonon mode $\gamma$, 


$$
\hat{H}_{\mathrm{at}-\mathrm{phn}}=\sum_{\gamma}\left[g_{\gamma}(\hat{\boldsymbol{r}}) \hat{b}_{\gamma}+\text { H.c. }\right],
$$

where $g_{\gamma}(\boldsymbol{r})=g_{\gamma}^{\mathrm{dp}}(\boldsymbol{r})+g_{\gamma}^{\mathrm{st}}(\boldsymbol{r})$. The coupling function $g_{\gamma}^{\mathrm{dp}}(\boldsymbol{r})$ derives from displacement coupling and $g_{\gamma}^{\text {st }}(\boldsymbol{r})$ from strain coupling.

Furthermore, we approximate the phonon-induced forces acting on a trapped atom as linear in the atom position by expanding Eq. (4) to first order around the trap minimum $\boldsymbol{r}_{0}$. The interaction Hamiltonian then takes the form [65]

$$
\hat{H}_{\mathrm{at}-\mathrm{phn}} \simeq \sum_{i \gamma} \hbar\left(\hat{a}_{i}+\hat{a}_{i}^{\dagger}\right)\left(g_{\gamma i} \hat{b}_{\gamma}+g_{\gamma i}^{*} \hat{b}_{\gamma}^{\dagger}\right)
$$

where the coupling constants are

$$
g_{\gamma i} \equiv \frac{\Delta x^{i}}{\hbar} \partial_{i} g_{\gamma}\left(\boldsymbol{r}_{0}\right)
$$

The length $\Delta x^{i} \equiv \sqrt{\hbar /\left(2 M \omega_{i}\right)}$ is the zero-point motion of the atom of mass $M$ in the trap. The coupling constants quantify the interaction of each phonon mode $\gamma$ with the motion of the atom in direction $i$. Analogous to the coupling function, there are contributions from both displacement and strain coupling $g_{\gamma i}=g_{\gamma i}^{\mathrm{dp}}+g_{\gamma i}^{\mathrm{st}}$.

The variation of the optical potential caused by displacement can in general be modeled by perturbatively calculating the new photonic eigenmodes in the presence of shifted boundaries of the nanostructure [66]. The displacement has two effects: First, it shifts the photonic structure, together with the electromagnetic fields surrounding it, relative to the trapped atom. Second, it deforms the surface of the structure, leading to new photonic eigenmodes and thereby also deforming the electromagnetic fields. The first effect scales with the ratio between the displacement of the surface and the size of the atom trap (the extent of the wave function of the atom). The second effect, on the other hand, scales with the ratio between the displacement and the dimensions of the structure. Since the trap is typically at least 1 order of magnitude smaller than the photonic structure (see Sec. III), we neglect the second effect and assume that both optical and surface potential are displaced as a whole together with the fiber surface [67]. This model is particularly useful for structures such as nanofibers which have a simple geometrical shape and highly symmetric mechanical modes. The resulting displacement coupling functions $g_{\gamma}^{\mathrm{dp}}(\boldsymbol{r})$ for a nanofiber-based atom trap in particular are given in Appendix D.

Strain leads to changes in the optical potential through the photoelastic effect, which can be modeled by a straindependent permittivity tensor $\overline{\boldsymbol{\epsilon}}[\boldsymbol{S}][34,68,69]$. The modified permittivity is then in general neither homogeneous nor isotropic and results in modified electric fields $\overline{\boldsymbol{E}}$ surrounding the fiber and thus in a modified optical potential $V_{\mathrm{opt}}[\overline{\boldsymbol{E}}]$. In consequence, the total potential $V[\boldsymbol{u}, \boldsymbol{S}]$ depends on strain. We neglect the influence of strain on the surface forces because they arise from the interaction of the atom with charges in a thin slice at the surface of the fiber and are largely independent of changes in the interior of the fiber [56]. The strain coupling function $g_{\gamma}^{\text {st }}(\boldsymbol{r})$ can then be obtained by perturbatively calculating the new photonic eigenmodes in the presence of a modified permittivity; see Appendix D.

Having obtained the Hamiltonian of the coupled atomphonon system, we can now describe the resulting evolution of the atomic motion. The cold atom can absorb kinetic energy from the thermally excited phonon field of the photonic structure (heating of the atomic motion). Provided that the atom-phonon coupling is weak compared to the trap frequencies and the coherence time of phonon excitations, the phonon field can be adiabatically eliminated. The effective evolution of the density matrix $\hat{\mu}(t)$ describing the motional state of the atom is then governed by a master equation [70,71]; see Appendix D. Heating of the atom is reflected in the increase of the expected number of motional quanta $n_{i}(t) \equiv \operatorname{tr}\left[\hat{\mu}(t) \hat{a}_{i}^{\dagger} \hat{a}_{i}\right]$ along a spatial direction $i$. The population grows linearly with heating rate $\Gamma_{i}^{\text {th }}$ for sufficiently short times

$$
n_{i}(t) \simeq \Gamma_{i}^{\mathrm{th}} t,
$$

assuming that the atom is in the motional ground state at $t=0$.

The phononic eigenmodes supported by the photonic structure can feature both discrete and continuous frequency spectra. Discrete spectra are observed for phonon modes with a spacing in frequency that is larger than their damping rates. In contrast, if a set of modes has frequency spacings much smaller than their damping rates (e.g., because the mechanical excitation is efficiently transmitted from the structure to its suspension), the discrete mechanical resonances are no longer discernible, and the spectrum is effectively continuous. Hence, we distinguish the contribution $\Gamma_{i}^{d}$ of discrete mechanical resonances from the contribution $\Gamma_{i}^{c}$ of a continuum of phonon modes:

$$
\Gamma_{i}^{\mathrm{th}}=\Gamma_{i}^{c}+\Gamma_{i}^{d} .
$$

For continuous phonon modes, Fermi's golden rule can be employed to calculate the heating rate $\Gamma_{i}^{c}[70]$ :

$$
\Gamma_{i}^{c}=2 \pi \bar{n}_{i} \sum_{\gamma_{i}} \rho_{\gamma_{i}}\left|g_{\gamma_{i} i}\right|^{2} .
$$

The sum runs over the discrete set of continuous phonon modes $\gamma_{i}$ that are resonant with the trap $\omega_{\gamma_{i}}=\omega_{i}$. The thermal occupation of the resonant phonon modes is $\bar{n}_{i} \equiv 1 /\left[\exp \left(\hbar \omega_{i} / k_{B} T\right)-1\right]$, where $T$ is the temperature of the photonic structure and $k_{B}$ is the Boltzmann 
constant [72]. The phonon density of states is given by the inverse slope of the phonon dispersion relation (band structure) $\rho_{\gamma} \equiv\left|d \omega_{\gamma} / d p\right|^{-1}$, where $p$ is the propagation constant along the fiber; see Appendix C.

The discrete resonances have finite lifetimes corresponding to decay rates $\kappa_{\gamma}$ due to internal losses and nonzero coupling to the suspension. Adiabatic elimination of these discrete mechanical modes in general leads to the heating rate $\Gamma_{i}^{d}$ given in Eq. (D40) in Appendix D $[73,74]$. There are two limiting cases that are of interest in Sec. III: In the case where the atom trap frequency is smaller than the lowest-frequency phonon mode $\gamma_{1}, \omega_{i}<\omega_{\gamma_{1}}$ and detuned from resonance $\kappa_{\gamma_{1}} \ll\left|\omega_{i}-\omega_{\gamma_{1}}\right|$, the ground-state heating rate of the atom is

$$
\Gamma_{i}^{d} \simeq 2 \bar{n} \kappa_{\gamma_{1}}\left|g_{\gamma_{1}}\right|^{2} \frac{\omega_{i}^{2}+\omega_{\gamma_{1}}^{2}}{\left(\omega_{i}^{2}-\omega_{\gamma_{1}}^{2}\right)^{2}} .
$$

In the case where the atom trap is resonant with a singlephonon mode $\gamma, \kappa_{\gamma} \gg\left|\omega_{i}-\omega_{\gamma}\right|$, the rate is

$$
\Gamma_{i}^{d} \simeq \frac{4 \bar{n}\left|g_{\gamma i}\right|^{2}}{\kappa_{\gamma}},
$$

where we assume $\bar{n} \gg 1$.

The theory of atom-phonon interaction outlined in this section applies to any optical atom trap that relies on a photonic structure to shape light fields. The explicit calculation of atom-phonon coupling constants requires modeling of the dependence of the potential that the atom experiences on the displacement and the strain caused by the mechanical eigenmodes of the structure. Once the mechanical modes and corresponding atom-phonon coupling constants of a particular structure are known, Eqs. (11)-(13), or more generally, Eq. (D4), can be used to predict the phonon-induced heating of the atomic motion. In the next section, we apply this theory to explain heating rates observed in nanofiber-based atom traps.

\section{CASE STUDY OF A NANOFIBER-BASED TRAP}

Let us now use the framework sketched in Sec. II to study the phonon-induced heating rates of the atomic motion in a nanofiber-based two-color atom trap. In particular, we consider a cesium atom trapped in the evanescent optical field surrounding a silica nanofiber $[47,49]$. The nanofiber is formed by the waist of an optical fiber which has been heated and pulled [75]. There have been several experimental realizations of this nanophotonic atom trap configuration $[4,5,7-9,24,26,76]$. We calculate atom heating rates for the setup described in Ref. [26], where a measured heating rate of $\Gamma_{\varphi}^{\text {th }}=340(10) \mathrm{Hz}$ in the azimuthal direction was reported. In order to explicitly calculate the phonon-induced heating rates, it is necessary to know the mechanical eigenmodes of the nanofiber close to resonance with the trap frequencies and to obtain the atomphonon coupling constants. The latter calculation requires knowledge of the trap potential as well as the photonic eigenmodes of the nanofiber. Appendix A summarizes the photonic eigenmodes of a nanofiber, and Appendix B provides details on the resulting trapping potential. Appendix $C$ summarizes the phononic eigenmodes. In Appendix D, we derive the resulting atom-phonon coupling constants for a nanofiber-based trap. The parameters of the particular setup considered in this section are listed in Appendix E.

Trapping of atoms is achieved by means of two lasers, one red and the other blue detuned with respect to the $D$ lines of cesium. The lasers are guided as photonic $\mathrm{HE}_{11}$ spatial modes in the nanofiber region; see Appendix A. Figure 4 in Appendix D shows the resulting trapping potential. The red-detuned laser is coupled into the fiber at both ends, leading to a standing wave that confines the atoms in the axial direction and creates a one-dimensional optical lattice. The laser beams are linearly polarized when coupled into the fiber, which leads to quasilinearly polarized fields with intensity maxima at opposite poles of the fiber cross section in the nanofiber region [77]. The corresponding electric field profiles are listed in Appendix E. The red- and blue-detuned field have orthogonal polarizations to obtain stronger azimuthal confinement [4]. There is an offset magnetic field oriented perpendicular to the fiber axis ( $z$ axis) along $z_{B}=\cos (\phi) \boldsymbol{e}_{x}+\sin (\phi) \boldsymbol{e}_{y}$, with $\phi=66^{\circ}$. Atoms are initially prepared in the Zeeman substate $F=4, M_{F}=-4$ of the hyperfine structure, where the offset magnetic field provides the quantization axis. The magnetic field causes a slight azimuthal shift of the trap sites. Nonetheless, the symmetry axes of the potential at the trap minimum are to a good approximation aligned with the radial, azimuthal, and axial unit vectors of a cylindrical coordinate system whose $z$ axis coincides with the nanofiber axis. We can therefore use $i \in\{r, \varphi, z\}$ for the atom trap directions in the atom Hamiltonian Eq. (2). The resulting frequencies of the atom trap are $\left(\omega_{r}, \omega_{\varphi}, \omega_{z}\right)=2 \pi \times(123,71.8,193) \mathrm{kHz}$.

An infinitely long nanofiber supports three phonon bands which do not have a cutoff at low frequencies: the torsional $\mathrm{T}_{01}$ band, longitudinal $\mathrm{L}_{01}$ band, and flexural $\mathrm{F}_{11}$ band; see Appendix C. Figure 6 shows the displacement of the nanofiber caused by phonon modes on each of these bands. The torsional band is linear and the longitudinal band asymptotically linear for low frequencies, with speeds of sound $c_{t}$ and $c_{h}$ introduced in Appendix C, respectively. The flexural band has a quadratic asymptote. The dispersion relations describing these bands as functions of the propagation constant $p$ are

$$
\omega_{T}=c_{t}|p|, \quad \omega_{L} \simeq c_{h}|p|, \quad \omega_{F} \simeq \frac{c_{h} R}{2} p^{2} .
$$

Here, $R$ is the radius of the nanofiber. These three fundamental bands are the only candidates for phonon-induced 
TABLE I. Atom-phonon coupling constants. Listed are the contributions of displacement (dp) and strain (st) coupling to the coupling constants. The displacement coupling constants $g_{\gamma i}^{\mathrm{dp}}$ are calculated according to Eq. (D6). The strain coupling constants $g_{\gamma i}^{\text {st }}$ are obtained from Eq. (8) with the coupling functions listed in Table XVI in Appendix D. Coupling to modes on the continuous $\mathrm{L}_{01}$ and $\mathrm{F}_{11}$ bands is independent of the position of the trap site along the fiber axis. In contrast, the strain coupling constants to the discrete $\mathrm{T}_{01}$ modes depend on the position since the torsional modes form standing waves, see Appendix C. Listed here are the maximal coupling constants; for radial motion, the coupling is maximal at the end of the nanofiber $(z=0, L)$, while it is maximal at the center of the nanofiber $(z=L / 2)$ for the azimuthal and axial motion.

\begin{tabular}{|c|c|c|c|c|c|c|}
\hline \multirow[b]{2}{*}{ Trap } & \multicolumn{2}{|c|}{$\mathrm{T}_{01}$} & \multicolumn{2}{|c|}{$\mathrm{L}_{01}$} & \multicolumn{2}{|c|}{$\mathrm{F}_{11}$} \\
\hline & $\left|g_{\gamma i}^{\mathrm{dp}}\right| / 2 \pi(\mathrm{Hz})$ & $\left|g_{\gamma i}^{\mathrm{st}}\right| / 2 \pi(\mathrm{Hz})$ & $\left|g_{\gamma i}^{\mathrm{dp}}\right| / 2 \pi(\mathrm{Hz} \sqrt{\mathrm{m}})$ & $\left|g_{\gamma i}^{\mathrm{st}}\right| / 2 \pi(\mathrm{Hz} \sqrt{\mathrm{m}})$ & $\left|g_{\gamma i}^{\mathrm{dp}}\right| / 2 \pi(\mathrm{Hz} \sqrt{\mathrm{m}})$ & $\left|g_{\gamma i}^{\mathrm{st}}\right| / 2 \pi(\mathrm{Hz} \sqrt{\mathrm{m}})$ \\
\hline$r$ & 0 & $5.47 \times 10^{-8}$ & $3.08 \times 10^{-9}$ & $1.56 \times 10^{-8}$ & $3.93 \times 10^{-4}$ & $2.18 \times 10^{-8}$ \\
\hline$\varphi$ & 0 & $7.81 \times 10^{-4}$ & 0 & $7.76 \times 10^{-11}$ & $2.28 \times 10^{-4}$ & $2.99 \times 10^{-10}$ \\
\hline$z$ & 0 & $2.19 \times 10^{-12}$ & 0 & $1.05 \times 10^{-4}$ & 0 & $1.13 \times 10^{-10}$ \\
\hline
\end{tabular}

heating of the atomic motion since all other bands have frequencies much larger than the trap frequencies.

In experiments, the optical nanofibers used for atom trapping are typically realized as the waist of a tapered optical fiber [4]. The mechanical eigenmodes of this system-including the nanofiber, the tapers, and the surrounding macroscopic fiber-can be calculated either analytically or using finite-element methods [78]. Since the fiber is finite in length, the eigenmodes are standing waves and the spectrum consists of discrete mechanical resonances. The system can in general support the same kinds of excitations as an infinite cylinder: torsional, longitudinal, and flexural. For some modes, the tapers act as reflectors and strongly localize them in the nanofiber region. Others are transmitted through the tapers and are delocalized over the entire fiber [78]. In practice, all modes are damped. Dissipation occurs, among others, due to clamping losses [79], friction with the background gas [34], material losses [80], and surface losses [81]. Depending on the magnitude of the damping $\kappa$ of each mode compared to the free spectral range (FSR), the actual spectrum ranges from discrete (FSR $\gg \kappa)$ to continuous (FSR $\ll \kappa)$. In the case of a discrete spectrum, standing waves of finite lifetime $1 / \kappa$ are a useful description of the mechanical dynamics of the fiber. In the limit of a continuous spectrum, the idealized eigenmodes of the system are no longer faithful representations, since the phonons interact too strongly with other d.o.f. and are dissipated before they can form standing waves. Instead, it is more useful to represent the phonons as propagating modes of an infinite structure which interact with the atom once and then never return (analogous to an atom interacting with fiber-guided or free-space photons). Some of the damping mechanisms can be modeled theoretically $[80,81]$. However, more reliable results are obtained by measuring damping rates for the particular fiber in use. We perform measurements of the mechanical modes of the particular nanofiber setup considered here [26] similar to Refs. [78,82]. While torsional resonances are clearly visible, there is no indication of resonantly enhanced longitudinal or flexural nanofiber modes.
The mode of lowest frequency is at $\omega_{T}=2 \pi \times 258 \mathrm{kHz}$ with a wavelength of $14.6 \mathrm{~mm}$ and a decay rate of $\kappa=$ $2 \pi \times 48(1) \mathrm{Hz}$. The torsional modes can be modeled faithfully by imposing hard boundary conditions on an elastic cylinder; see Ref. [78] and Appendix C. The resulting spectrum is a discrete subset of the $\mathrm{T}_{01}$ band of an infinite cylinder. In keeping with the absence of discrete resonances corresponding to longitudinal and flexural modes, we model these modes as the propagating modes of an infinite cylinder, with a continuous dispersion relation given by the longitudinal and flexural bands Eq. (14). The form of the longitudinal and flexural mechanical bands and the corresponding eigenmodes are then determined by the elastic mechanical properties of silica and the fiber radius alone. The wavelengths of the modes resonant with the azimuthal trap frequency, for instance, are $80.0 \mathrm{~mm}$ for the $\mathrm{L}_{01}$ mode and $0.251 \mathrm{~mm}$ for the $\mathrm{F}_{11}$ mode.

The theory derived in Sec. II allows us to calculate atom heating rates based on these physical parameters. The only parameter not provided by Ref. [26] is the fiber temperature $T$. We choose the temperature such that the azimuthal heating rate $\Gamma_{\varphi}^{\text {th }}$ observed in Ref. [26] is reproduced. Agreement with the measurement in Ref. [26] is achieved for $T=805 \mathrm{~K}$, which agrees well with the temperature of $T=850(150) \mathrm{K}$ measured independently in Ref. [55] for a similar nanofiber at the given transmitted laser power. Heating in the azimuthal direction is dominantly caused by resonant flexural $\mathrm{F}_{11}$ modes. To our knowledge, this is the first time that a theoretical prediction of the atom heating rate based on measured parameters and in quantitative agreement with measured heating rates has been obtained. We are then able to calculate the phonon-induced heating rates of the atomic motion in the radial, azimuthal, and axial direction accounting for both displacement and strain coupling. The predicted atom-phonon coupling constants are listed in Table I and the resulting heating rates in Table II.

The predicted heating rate for the radial d.o.f. is a magnitude similar to the rate for the azimuthal d.o.f. The calculated radial heating rate is $\Gamma_{r}^{\text {th }}=446 \mathrm{~Hz}$, which 

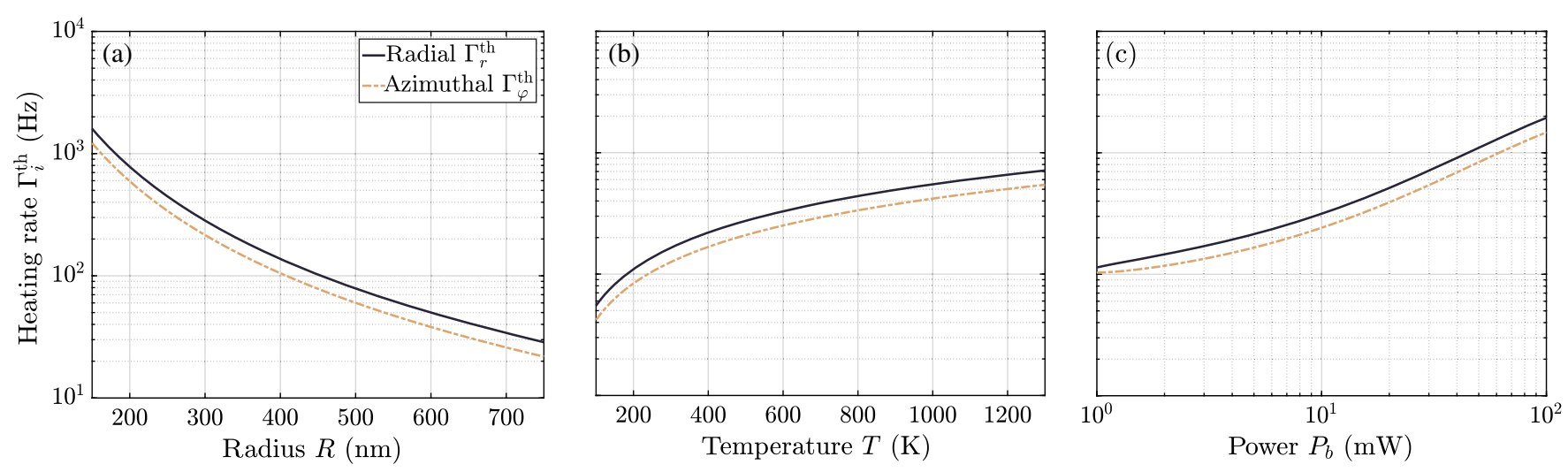

FIG. 1. Atom heating rate in the radial and azimuthal direction calculated using Eq. (15) as function of (a) the nanofiber radius, (b) the temperature of the nanofiber, and (c) the power of the blue-detuned trapping laser. The difference between Eq. (15) and the full theory Eq. (10) is not discernible at the given scales. In (a) and (b), all other parameters, in particular, the trap frequencies, are unchanged. In (c), the ratio between the power of the red- and blue-detuned laser is kept constant, $P_{b} / P_{r}=14.24$. The relation between the total laser power and temperature is modeled as $T(P)=m_{0}+m_{1} P+m_{2} P^{2}$, with $m_{0}=400 \mathrm{~K}, m_{1}=24 \mathrm{~K} / \mathrm{mW}, m_{2}=-0.062 \mathrm{~K} / \mathrm{mW}^{2}$ based on the measurements in Ref. [55] for a nanofiber of radius $R=250 \mathrm{~nm}$ and length $L=5 \mathrm{~mm}$. The temperature then varies from $T=427$ to $2298 \mathrm{~K}$ over the shown range of laser power. The trap frequencies simultaneously increase from $\left(\omega_{r}, \omega_{\varphi}\right)=2 \pi \times$ $(29.1,23.9) \mathrm{kHz}$ to $2 \pi \times(291,168) \mathrm{kHz}$. The remaining parameters are specified in Appendix E.

agrees with the heating rate assumed in Ref. [25] to explain measured $T_{2}^{\prime}$ decoherence rates for nanofiber-trapped atoms. Heating along the radial axis, like heating in the azimuthal direction, is dominated by coupling to the resonant flexural $F_{11}$ modes. The coupling constants in Table I reveal that the coupling is due to displacement of the fiber surface, while coupling due to strain is lower by several orders of magnitude. A priori, both longitudinal $\mathrm{L}_{01}$ and flexural $\mathrm{F}_{11}$ modes couple to the radial motion by displacement. However, the flexural modes lead to much higher heating rates for two reasons: First, flexural modes displace the fiber surface by a factor of $\left|w_{F}^{r} / w_{L}^{r}\right| \simeq$ $\sqrt{E /(2 \rho)} /\left(\omega_{r} \nu R\right) \simeq 10^{5}$ more than the longitudinal modes, which leads to larger displacement coupling constants. Here, $w_{F}^{r}$ and $w_{L}^{r}$ are the radial components of the displacement eigenmode for the flexural and longitudinal modes, respectively. The quantity $E$ is Young's modulus and $\nu$ is the Poisson ratio; together, they describe the elastic properties of the nanofiber. The quantity $\rho$ is the mass density of the nanofiber and $\omega_{r}$ the radial trap frequency. The second reason is that the density of states of the flexural modes is larger than the one of longitudinal modes by a factor of $\rho_{F r} / \rho_{L} \simeq \sqrt{c_{h} /\left(2 \omega_{r} R\right)} \simeq 100$, and the heating rates are enhanced accordingly; see Eq. (11).

Heating in the axial direction is predicted to be predominantly due to strain coupling to the resonant longitudinal $\mathrm{L}_{01}$ mode, with a rate much smaller than the heating rates in the radial and azimuthal direction. To the best of our knowledge, the heating rate in the axial direction has not been measured so far.

One might expect heating by near-resonant torsional modes to be dominant because they are tightly confined to the nanofiber region, leading to Purcell enhancement of the coupling strength [72]. The strain induced by torsional modes causes a tilt of the quasilinear polarization of the light fields (see Fig. 7 in Appendix D), which leads to coupling to the azimuthal motion of the atom in particular. In the present case, the contribution of torsional modes to the heating is negligible due to the large detuning between the torsional mode and trap frequencies compared to the phonon decay rate. However, we can use Eq. (13) with the coupling constants given in Table I to obtain an estimate of the heating rates expected in case the torsional modes are resonant (e.g., in case the nanofiber is longer). In this worst-case scenario, the predicted contribution to the heating rate in the azimuthal direction is $\Gamma_{\varphi}^{d}=17.8 \mathrm{~Hz}$, while heating in the other trap directions is still below $10^{-4} \mathrm{~Hz}$ despite the Purcell enhancement. For the hypothetical case in which the torsional modes are not reflected at the ends of the nanofiber, our model predicts even lower heating rates. Hence, torsional modes are not a relevant source of heating in Ref. [26], even if they are resonant with the trap frequencies.

In summary, the atom heating in the radial and azimuthal direction observed in experiments is well explained by the displacement coupling to the continuous $F_{11}$ band alone. In this case, Eq. (11) simplifies to the single equation

$$
\Gamma_{i}^{\mathrm{th}} \simeq \frac{1}{2 \sqrt{2} \pi} \frac{k_{B}}{\hbar} T M \sqrt{\frac{\omega_{i}}{R^{5} \sqrt{E \rho^{3}}}}, \quad i \in\{r, \varphi\},
$$

where we use that $\hbar \omega_{i} \ll k_{B} T$, such that the thermal occupation of the phonon modes is $\bar{n}_{i} \simeq k_{B} T / \hbar \omega_{i}$. This simple formula agrees exceedingly well with calculations considering all phonon modes and both displacement and strain coupling. Figure 1 shows the dependence of the predicted heating rates in the radial and azimuthal direction 

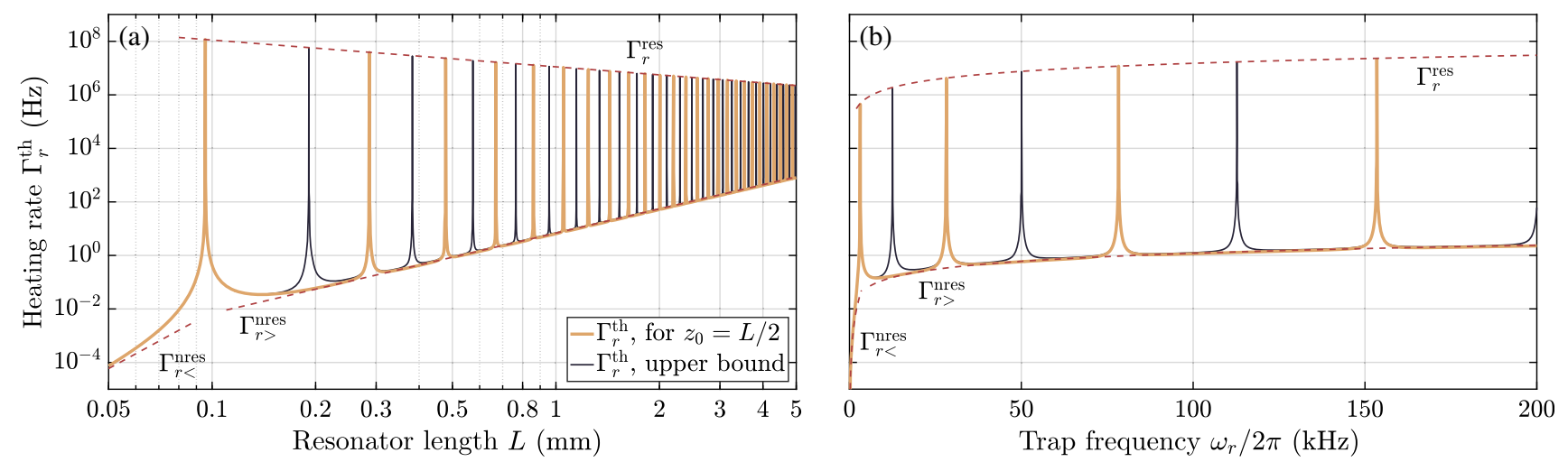

FIG. 2. Atom heating rate in the radial direction due to flexural resonator modes as a function of (a) the resonator length and (b) the trap frequency. In both cases, an exemplary decay rate of $\kappa=2 \pi \times 1.2 \mathrm{~Hz}$ is assumed for all resonator modes. The bold yellow line corresponds to the heating rate experienced by an atom trapped at the center of the resonator $z_{0}=L / 2$ calculated according to Eq. (D41). The thin blue line represents a position-independent upper bound obtained by pretending that the atom sits at an antinode of each phonon mode simultaneously: In consequence, no resonance between the atom and resonator is masked by a vanishing positiondependent coupling rate. This approach is useful, since in experiments an entire ensemble of atoms is trapped at various positions along the fiber. The dashed red lines show the approximations Eqs. (17)-(19). Panel (a) assumes a trap frequency of $\omega_{r}=2 \pi \times 123 \mathrm{kHz}$ and panel (b) assumes a resonator length of $L=600 \mu \mathrm{m}$.

on individual parameters, keeping the remaining parameters unchanged. Most pronounced is the scaling with the nanofiber radius as $\Gamma_{i}^{\text {th }} \propto R^{-5 / 2}$; see Fig. 1(a). The strong dependence on the radius is mostly due to the increased mechanical stability of larger nanofibers, which leads to smaller vibrational amplitudes [see Eq. (C34)], in addition to a lower density of states. In contrast, the dependence on the fiber temperature is linear [see Fig. 1(b)] since the thermal occupation of the resonant phonon modes increases linearly with the temperature. A comparison of Figs. 1(a) and 1 (b) shows that increasing the nanofiber radius by $150 \mathrm{~nm}$ to $R=400 \mathrm{~nm}$ at constant temperature has an effect comparable to cooling the fiber down to room temperature if all other parameters of the setup can be kept unchanged. Figure 1(c) shows the dependence on the power of the blue-detuned laser, where the ratio of the power of the red- and blue-detuned lasers is kept constant. The temperature of the nanofiber increases with increased laser power since there is more absorption in the fiber [55]; see caption for details. Moreover, higher intensities lead to a tighter confinement of the atoms. The observed increase of the heating rate when raising the laser power is therefore caused by an increase of both the fiber temperature and the trap frequencies. While Young's modulus $E$ also slightly changes with $T$ [83], the influence of this effect on the heating rate is negligible due to the weak dependence, $\Gamma_{i}^{\text {th }} \propto E^{-1 / 4}$.

Let us now discuss ways to reduce the atom heating caused by coupling to the continuous $\mathrm{F}_{11}$ band. Lowering the overall fiber temperature in order to reduce the heating rates is difficult even in cryogenic environments because thermal coupling of the fiber to its surroundings is very weak [55]. However, based on the above analysis, different strategies to minimize the heating rates are conceivable.
First of all, the fiber radius should be chosen as large as possible while maintaining the optical properties required for atom trapping. A second approach is to design the nanofiber such that it supports discrete, well-resolved resonances of flexural modes. While precise predictions of phonon linewidths are difficult, it may be possible to optimize the taper at both ends of the nanofiber and ensure that flexural modes are reflected and confined to $z \in[0, L]$ with narrow linewidths, while the transmission of light is not reduced [79]. Such a resonator of length $L$ for the flexural modes would effectively break the $\mathrm{F}_{11}$ band into a discrete set of frequencies $\omega_{m}$ and allow us to detune the atom trap from resonance with these mechanical modes. The flexural eigenmodes are then standing waves (see Appendix C) with frequency spectrum

$$
\omega_{m} \equiv m^{2} \frac{\pi^{2} R}{2 L^{2}} \sqrt{\frac{E}{\rho}}, \quad m \in \mathbb{N} .
$$

The heating rate in the radial and azimuthal direction due to these flexural resonator modes then depends on the position $z_{0}$ of the atom along the fiber axis; see Appendix D. Figure 2 shows the dependence of the heating rate on the resonator length and trap frequency. Three regimes are clearly distinguishable: First, the trap is resonant with a flexural phonon mode. Second, the trap is off resonant and lies below the fundamental resonator frequency. Third, the trap is off resonant and lies above the fundamental resonator frequency. Assuming high thermal occupation of the phonon modes $\bar{n}_{m} \gg 1$, simplified expressions for the heating rate can be obtained for each regime. If the trap frequency is below the fundamental phonon frequency but still much larger than the corresponding decay rate 
$\kappa_{1} \ll \omega_{i}<\omega_{1}$, as well as far detuned $\left|\omega_{i}-\omega_{1}\right| \gg \kappa_{1}$, heating is dominated by off-resonant interaction with the fundamental phonon mode alone. In this case, the heating rate can be approximated as

$$
\begin{aligned}
\Gamma_{i}^{\text {th }} & \simeq \Gamma_{i<}^{\text {nres }} \sin ^{2}\left(\pi z_{0} / L\right), \\
\Gamma_{i<}^{\text {nres }} & \equiv \frac{16}{\pi^{9}} \frac{k_{B}}{\hbar} \frac{T M \rho \kappa_{1} \omega_{i}^{3} L^{7}}{E^{2} R^{6}} .
\end{aligned}
$$

If the trap has a frequency larger than the fundamental resonator frequency $\omega_{i} \gg \omega_{1}$ while still being off resonant $\left|\omega_{i}-\omega_{m}\right| \gg \kappa_{m}$, heating is mainly due to the lowfrequency phonon modes below the trap frequency. Assuming in addition that the phonon decay rate is the same for all relevant modes $\kappa_{m} \simeq \kappa$, an upper bound for the heating rate can be obtained:

$$
\Gamma_{i}^{\mathrm{th}} \lesssim \Gamma_{i>}^{\mathrm{nres}} \equiv \frac{2}{45 \pi} \frac{k_{B}}{\hbar} \frac{T M \kappa \omega_{i} L^{3}}{E R^{4}} .
$$

Here, we replace the sine in the coupling constant with 1 for all modes, pretending the atom is located at an antinode of all modes simultaneously as a worst-case estimate. This approximation is useful because in experiments many atoms at different sites along the fiber axis are trapped at the same time.

If the trapped atom is resonant with a flexural phonon mode $m,\left|\omega_{i}-\omega_{m}\right| \ll \kappa_{m}$, and the contributions of the offresonant modes can be neglected, the heating rate is

$$
\begin{aligned}
\Gamma_{i}^{\mathrm{th}} & \simeq \Gamma_{i}^{\mathrm{res}} \sin ^{2}\left(p_{m} z_{0}\right), \\
\Gamma_{i}^{\mathrm{res}} & \equiv \frac{2}{\pi} \frac{k_{B}}{\hbar} \frac{T M \omega_{i}}{L \rho \kappa_{m} R^{2}} .
\end{aligned}
$$

The limiting expressions Eqs. (17)-(19) are shown as dashed black lines in Fig. 2. Note that the dependence on the decay rate and resonator length is inverted for offresonant heating [Eqs. (17) and (18)] compared to resonant heating [Eq. (19)]. This inversion is expected, since large phonon linewidths $\kappa_{m}$ assist off-resonant coupling, while small linewidths lead to a larger resonant enhancement. Small resonator lengths $L$ lead to higher coupling constants (Purcell enhancement), which increases resonant heating due to a single mode. In contrast, large resonator lengths result in a higher number of low-frequency modes and hence overcompensate the decrease in coupling strength and increase the heating due to off-resonant interaction.

In Fig. 2, we exemplarily assume a decay rate of $\kappa_{m}=$ $2 \pi \times 1.2 \mathrm{~Hz}$ for all relevant flexural modes. This corresponds to a quality factor of $\omega_{r} / \kappa_{m}=10^{5}$ at the frequency of the radial trap. Quality factors of this magnitude have been achieved for silica microspikes by optimization of the shape of the taper [79]. Figure 2(a) shows that a decrease of the radial heating rate below the value expected without a resonator for flexural modes (see Table II) is predicted for
TABLE II. Atom heating rates. Listed are the contributions of the relevant phonon modes $\mathrm{T}_{01}, \mathrm{~L}_{01}$, and $\mathrm{F}_{11}$ to the heating rate $\Gamma_{i}^{\text {th }}$ of a trapped atom in direction $i \in\{r, \varphi, z\}$ calculated according to Eqs. (11) and (12). Contributions below $10^{-4} \mathrm{HZ}$ are indicated by "«." The rates are independent of the position of the trap site along the fiber. The fiber temperature is assumed to be $T=805 \mathrm{~K}$, and the remaining parameters are specified in Appendix E.

\begin{tabular}{lccc}
\hline \hline Trap & $\mathrm{T}_{01}$ & $\mathrm{~L}_{01}$ & $\mathrm{~F}_{11}$ \\
\hline$r$ & $\ll$ & $\ll$ & $446 \mathrm{~Hz}$ \\
$\varphi$ & $\ll$ & $\ll$ & $340 \mathrm{~Hz}$ \\
$z$ & $\ll$ & $8.36 \times 10^{-2} \mathrm{~Hz}$ & $\ll$ \\
\hline \hline
\end{tabular}

resonator lengths $L \lesssim 3 \mathrm{~mm}$. A length of $L=50 \mu \mathrm{m}$ to the very left of Fig. 2(a) can still be achieved for nanofibers, and the calculated heating rate due to flexural phonon modes with the given decay rate is then as low as $0.1 \mathrm{mHz}$. Figure 2(b) assumes a resonator length of $L=600 \mu \mathrm{m}$, achieving heating rates of around $1 \mathrm{~Hz}$ and shows the dependence on the trap frequency. The spacing between resonances is on the order of $2 \pi \times 50 \mathrm{kHz}$, which would indeed render it possible to detune the radial and azimuthal trap from resonance.

These findings suggest that it may be possible to significantly reduce the heating rate of atomic motion in nanofiber-based traps by 2 orders of magnitude or more through optimization of the phononic properties of the fiber. Moreover, the scaling of the heating rate with the mass of the trapped particles as $\Gamma_{i}^{\text {th }} \propto M$ is highly relevant for optomechanical experiments. Setups with levitated nanoparticles, for instance, may feature comparable trap frequencies for particles that are orders of magnitude heavier than a single atom $[3,12]$. In order to stably trap heavier particles using nanophotonic structures and successfully cool their motion, it is imperative to carefully manage vibrations of the structure, for instance, by improving the mechanical stability or by tuning mechanical modes out of resonance with the particle motion.

\section{CONCLUSION}

In this article, we formulate a general theoretical framework for calculating the effect of phonons on guided optical modes and the resulting heating of atoms in nanophotonic traps. Our results are applicable to nanophotonic cold-atom systems [45] and can readily be extended to the heating of dielectric nanoparticles trapped close to surfaces [3,12]. In a case study for the example of cold cesium atoms in a twocolor nanofiber-based optical trap, we predict heating rates of the atomic center-of-mass motion which are in excellent agreement with independently measured values $[25,26]$. In this system, the dominant contribution to heating stems from thermally occupied flexural modes of the nanofiber. We find that the heating rate scales with the fiber radius as 
$R^{-5 / 2}$. As a general design rule, this implies that structures of larger lateral dimensions are preferable regarding heating, albeit at the expense of smaller mode confinement and, hence, potentially lower atom-photon coupling strength. Given the fact that the heating rate is directly proportional to the temperature of the nanophotonic structure, reducing the absorption losses of the guided trapping light fields is advisable [84]. Moreover, heating is expected to decrease for smaller trap frequencies $\Gamma \propto \sqrt{\omega}$. In general, our case study shows that careful design of the phononic properties of the nanophotonic system and, in particular, of its mechanical resonances is an effective strategy for reducing the heating. Finally, by providing a coherent theoretical framework in a single source, our work is instrumental in calculating, understanding, and managing heating in a plethora of nanophotonic traps.

\section{ACKNOWLEDGMENTS}

We thank Y. Meng for the experimental characterization of the torsional mode resonances of the tapered optical fiber in the nanofiber-based two-color trap setup. Financial support by the European Research Council (CoG NanoQuaNt) and the Austrian Academy of Sciences (ÖAW, ESQ Discovery Grant QuantSurf) is gratefully acknowledged. We acknowledge support by the Austrian Federal Ministry of Science, Research, and Economy (BMWFW).

\section{APPENDIX A: PHOTONIC EIGENMODES}

The potential experienced by an atom in a nanophotonic trap crucially depends the optical fields surrounding the photonic structure. The dynamics of optical fields in the presence of nonabsorbing matter is well described by the macroscopic Maxwell equations. In conjunction with linear response theory, they allow us to model materials using the relative permittivity and permeability tensors $\boldsymbol{\epsilon}$ and $\boldsymbol{\mu}$, respectively [85]. In this article, we consider dielectric materials that are not magnetizable $(\boldsymbol{\mu}=\mathbb{1})$. Motion and vibration of the dielectric can be modeled as a change of $\boldsymbol{\epsilon}$ over time, provided this change happens on a timescale long compared to the frequency of electromagnetic radiation in the optical regime. With this in mind, we choose a description of the optical fields in terms of photonic eigenmodes [86], which lends itself well to a perturbative treatment of the effect of a modified permittivity on the optical fields [87] as we discuss in Appendix D.

After reviewing photonic eigenmodes in general, we describe the eigenmode structure of a nanofiber approximated as a homogeneous step-profile circular optical waveguide [87-89].

\section{Photonic eigenmode equation}

Consider a dielectric body in three dimensions. The body may be inhomogeneous and anisotropic, so its relative permittivity $\boldsymbol{\epsilon}$ is a position-dependent tensor of second order. We assume that the permittivity is independent of frequency in the relevant interval, real-valued, symmetric, and positive definite. In the vacuum outside the body, $\boldsymbol{\epsilon}=\mathbb{1}$. We are interested in the dynamics of the electromagnetic fields $\boldsymbol{E}$ and $\boldsymbol{B}$ surrounding and permeating the dielectric. We express the electromagnetic fields through potentials and follow Ref. [90] in choosing the Coulomb gauge for the vector potential $\boldsymbol{A}$,

$$
\nabla \cdot[\boldsymbol{\epsilon}(\boldsymbol{r}) \boldsymbol{A}(\boldsymbol{r}, t)]=0 .
$$

Here, the juxtaposition of tensor and vector (or, more generally, of two tensors) indicates the maximal contraction $(\boldsymbol{\epsilon A})^{i}=\sum_{j} \epsilon^{i j} A^{j}$. In the absence of free charges, the electromagnetic fields can be represented solely through the vector potential $\boldsymbol{E}=\dot{\boldsymbol{A}}$ and $\boldsymbol{B}=\nabla \times \boldsymbol{A}$. The macroscopic Maxwell equations reduce to

$$
\frac{1}{c^{2}} \boldsymbol{\epsilon}(\boldsymbol{r}) \ddot{\boldsymbol{A}}(\boldsymbol{r}, t)=\mathcal{D} \boldsymbol{A}(\boldsymbol{r}, t),
$$

where $\mathcal{D} \equiv-\nabla \times[\nabla \times \cdot]$ is the double curl operator, each dot represents a time derivative $\dot{\boldsymbol{A}}=\partial_{t} \boldsymbol{A}$, and $c$ is the vacuum light speed. In order to find solutions, one solves Eq. (A2) outside and inside the body separately and then uses continuity conditions to match the solutions at the interface: The magnetic field $\boldsymbol{B}$ as well as the electric field component $\boldsymbol{E} \times \boldsymbol{n}$ orthogonal to the surface normal $\boldsymbol{n}$ are continuous across the surface. Normal to the surface, $(\boldsymbol{E} \boldsymbol{E}) \cdot \boldsymbol{n}$ is continuous instead. Equation (A2), together with the continuity conditions and the requirement that solutions be square integrable to ensure finite electromagnetic energy, has a unique solution given suitable initial conditions [85].

The above problem can be further reduced to an eigenvalue problem $[86,90,91]$, which is useful for describing phonon-induced perturbations of optical fields in Appendix D. To this end, consider the generalized eigenvalue equation for photonic eigenmodes $\boldsymbol{a}_{\eta}$,

$$
\mathcal{D} \boldsymbol{a}_{\eta}(\boldsymbol{r})=-\frac{\omega_{\eta}^{2}}{c^{2}} \boldsymbol{\epsilon}(\boldsymbol{r}) \boldsymbol{a}_{\eta}(\boldsymbol{r})
$$

with the additional transversality constraint Eq. (A1). The eigenmodes are labeled by a suitable multi-index $\eta$ which may contain both discrete and continuous indices. They span a subspace characterized by Eq. (A1) of the space of square-integrable functions [90]. The eigenvalues $\omega_{\eta}^{2} / c^{2}$ are real and positive, since $(-\mathcal{D})$ acting on that space is a self-adjoint, positive semidefinite operator, and $\boldsymbol{\epsilon}$ is a positive definite operator [86]. For the same reason, different eigenmodes are orthogonal with respect to the measure $\boldsymbol{\epsilon}(\boldsymbol{r}) d r$, and we assume that they are normalized according to 


$$
\int \boldsymbol{a}_{\eta}^{*}(\boldsymbol{r}) \cdot\left[\boldsymbol{\epsilon}(\boldsymbol{r}) \boldsymbol{a}_{\eta^{\prime}}(\boldsymbol{r})\right] d \boldsymbol{r}=\delta_{\eta \eta^{\prime}}
$$

For discrete indices, $\delta$ is the Kronecker symbol, while it is the $\delta$ distribution for continuous indices.

Any solution to Maxwell's equations can then be expanded in terms of eigenmodes of well-defined frequencies [92]

$$
\boldsymbol{A}(\boldsymbol{r}, t)=\sum_{\eta} \frac{1}{\omega_{\eta} \epsilon_{0}}\left[\alpha_{\eta} e^{-i \omega_{\eta} t} \boldsymbol{a}_{\eta}(\boldsymbol{r})+\text { c.c. }\right],
$$

where the coefficients $\alpha_{\eta} \in \mathbb{C}$ are obtained from the initial conditions. We define the modal fields of the electric and magnetic field as

$$
\boldsymbol{e}_{\eta}(\boldsymbol{r}) \equiv \frac{i}{\epsilon_{0}} \boldsymbol{a}_{\eta}(\boldsymbol{r}), \quad \boldsymbol{b}_{\eta}(\boldsymbol{r}) \equiv \frac{1}{\omega_{\eta} \epsilon_{0}} \nabla \times \boldsymbol{a}_{\eta}(\boldsymbol{r})
$$

for convenience, such that

$$
\begin{aligned}
& \boldsymbol{E}(\boldsymbol{r}, t)=\sum_{\eta}\left[\alpha_{\eta} \boldsymbol{e}_{\eta}(\boldsymbol{r}) e^{-i \omega_{\eta} t}+\text { c.c. }\right], \\
& \boldsymbol{B}(\boldsymbol{r}, t)=\sum_{\eta}\left[\alpha_{\eta} \boldsymbol{b}_{\eta}(\boldsymbol{r}) e^{-i \omega_{\eta} t}+\text { c.c. }\right] .
\end{aligned}
$$

The problem of solving Maxwell's equations in the presence of a dielectric body has therefore been reduced to finding the photonic eigenmodes $\boldsymbol{a}_{\eta}$ of that body. Although it is sufficient to treat the optical fields classically for our purpose, note that such a description is also suitable for canonical quantization of the electromagnetic field in the presence of lossless media $[90,91]$.

\section{Photonic fiber eigenmodes}

We now consider an optical nanofiber in vacuum modeled as a cylinder of radius $R$, infinite length, and homogeneous and isotropic permittivity $\boldsymbol{\epsilon}=\epsilon \mathbb{1}$. As the eigenmodes of such a fiber and their spectrum are well known [87-89,93], we limit the discussion to their salient properties and list explicit expressions for the electric and magnetic modal fields as well as their dispersion relations.

We choose cylindrical coordinates $(r, \varphi, z)$, with $x=r \cos \varphi, y=r \sin \varphi$, and the $z$ axis coinciding with the fiber axis. We do not solve the generalized eigenvalue equation (A3) directly in order to obtain the eigenmodes of the vector potential. Instead, we remain on the level of electric and magnetic fields and solve the macroscopic Maxwell equations for constant permittivity $\epsilon$ in the spectral domain,

$$
\begin{aligned}
\nabla \cdot \boldsymbol{e}(\boldsymbol{r}) & =0, \quad \nabla \cdot \boldsymbol{b}(\boldsymbol{r})=0, \\
\nabla \times \boldsymbol{b}(\boldsymbol{r}) & =-i \frac{\omega}{v^{2}} \boldsymbol{e}(\boldsymbol{r}), \quad \nabla \times \boldsymbol{e}(\boldsymbol{r})=i \omega \boldsymbol{b}(\boldsymbol{r}) .
\end{aligned}
$$

TABLE III. Definitions of the radial constants $a$ and $b$, as well as the dimensionless quantities appearing in the photon modal fields. The definitions are given in terms of the azimuthal order $m$, propagation constant $k$, frequency $\omega$, radial position $r$, fiber radius $R$, and the light speed $c$ in vacuum and $v \equiv c / \sqrt{\varepsilon}$ in the fiber, respectively.

\begin{tabular}{rlrl}
\hline \hline$a$ & $=\sqrt{\omega^{2} / v^{2}-k^{2}}$ & $\tilde{a}$ & $=-i a$ \\
$b$ & $=\sqrt{\omega^{2} / c^{2}-k^{2}}$ & $\tilde{b}$ & $=-i b$ \\
$\omega_{v}$ & $=v|k|$ & $\omega_{c}$ & $=c|k|$ \\
$\alpha$ & $=a R$ & $\tilde{\alpha}$ & $=\tilde{a} R$ \\
$\beta$ & $=b R$ & $\tilde{\beta}$ & $=\tilde{b} R$ \\
$\kappa$ & $=k R$ & $x$ & $=r / R$ \\
$w$ & $=\omega R / c$ & $\eta$ & $=J_{m}(\alpha) / K_{m}(\tilde{\beta})$ \\
$\gamma$ & $=\kappa w(\varepsilon-1) J_{m}(\alpha) K_{m}(\tilde{\beta})$ & & \\
& $\times\left\{\alpha \tilde{\beta}\left[\alpha J_{m}(\alpha) K_{m}^{\prime}(\tilde{\beta})+\tilde{\beta} J_{m}^{\prime}(\alpha) K_{m}(\tilde{\beta})\right]\right\}^{-1}$ \\
$\sigma_{k}$ & $=k /|k|$ & $\sigma_{m}$ & $=m /|m|$ \\
\hline \hline
\end{tabular}

Here, $v \equiv c / \sqrt{\epsilon}$ inside the fiber, and $v$ is replaced with $c$ outside the fiber. Equation (A8) can be solved in vacuum and in the dielectric separately. Both sets of solutions are then matched on the fiber surface according to the continuity conditions given above to find the modal fields $\boldsymbol{e}_{\eta}$ and $\boldsymbol{b}_{\eta}$. The eigenmodes $\boldsymbol{a}_{\eta}$ can be obtained by inverting Eq. (A6).

The first step is to solve Eq. (A8) in the presence of an infinite isotropic medium of arbitrary homogeneous relative permittivity $\epsilon>0$ (including vacuum $\epsilon=1$ and dielectric $\epsilon>1$ ). The solution space is spanned by electric and magnetic fields of the form

$$
\begin{aligned}
\boldsymbol{e}(\boldsymbol{r}) & =\frac{\mathcal{E}(r)}{2 \pi} e^{i(m \varphi+k z)}, \\
\boldsymbol{b}(\boldsymbol{r}) & =\frac{\mathcal{B}(r)}{2 \pi} e^{i(m \varphi+k z)} .
\end{aligned}
$$

The propagation constant $k \in \mathbb{R}$ labels continuous excitations along the fiber axis, and the azimuthal order $m \in \mathbb{Z}$ discrete excitations in the azimuthal direction. The radial partial waves $\mathcal{E}$ and $\mathcal{B}$ depend on the magnitude of the frequency $\omega$ compared to the light line $\omega_{v} \equiv v|k|$ ( $v=c$ for $\epsilon=1)$ as well as other quantities defined in Table III. The radial partial waves are given in Table IV.

In the second step, a first set of solutions $\boldsymbol{e}, \boldsymbol{b}$ inside the fiber (with permittivity $\epsilon>1$, light speed $v \equiv c / \sqrt{\epsilon}$, and dielectric radial constant $a$ defined in Table III) is matched to a second set $\tilde{\boldsymbol{e}}, \tilde{\boldsymbol{b}}$ of solutions outside the fiber (with $\epsilon=1$, light speed $c$, and vacuum radial constant $b$ defined in Table III). The continuity conditions require $\epsilon e^{r}=\tilde{e}^{r}$ on the fiber surface, while the magnetic field and the remaining two components of the electric field need to be continuous. These conditions lead to electric and magnetic modal fields $\boldsymbol{e}_{\eta}, \boldsymbol{b}_{\eta}$ together with frequency equations governing their eigenfrequency $\omega_{\eta}$. Mode quadruplets 
TABLE IV. Radial partial waves of the solutions Eq. (A9) to Maxwell's equations using cylindrical coordinates in the spectral domain. Inside the fiber, $\omega_{0}=\omega_{v}$. In vacuum outside the fiber, $\omega_{0}=\omega_{c}$, and $\epsilon=1$ such that $\alpha$ is replaced with $\beta$. The quantities $A, B, C, D \in \mathbb{C}$ are amplitudes. The radial dependence is given by Bessel functions $J_{m}, Y_{m}$ above the light line, by modified Bessel functions $I_{m}, K_{m}$ below the light line, and by polynomials and the natural logarithm $\ln$ on the light line. The prime indicates the first derivative $J_{m}^{\prime}(x)=\partial_{x} J_{m}(x)$. All other quantities used are defined in Table III.

\section{Case}

(1) $\omega>\omega_{0} m \in \mathbb{Z}$

(2a) $\omega=\omega_{0} m=0$

(2b) $\omega=\omega_{0}|m|=1$

(2c) $\omega=\omega_{0}|m|=2$

(3) $\omega<\omega_{0} m \in \mathbb{Z}$

\section{Solution}

$$
\begin{aligned}
& \mathcal{E}^{r}=i \alpha^{-2}\left\{\kappa \alpha\left[A J_{m}^{\prime}(\alpha x)+B Y_{m}^{\prime}(\alpha x)\right]+i m w\left[C J_{m}(\alpha x)+D Y_{m}(\alpha x)\right] / x\right\} \\
& \mathcal{E}^{\varphi}=i \alpha^{-2}\left\{i m \kappa\left[A J_{m}(\alpha x)+B Y_{m}(\alpha x)\right] / x-w \alpha\left[C J_{m}^{\prime}(\alpha x)+D Y_{m}^{\prime}(\alpha x)\right]\right\} \\
& \mathcal{E}^{z}=A J_{m}(\alpha x)+B Y_{m}(\alpha x) \\
& \mathcal{B}^{r}=i \alpha^{-2}\left\{\kappa \alpha\left[C J_{m}^{\prime}(\alpha x)+D Y_{m}^{\prime}(\alpha x)\right]-i m \epsilon w\left[A J_{m}(\alpha x)+B Y_{m}(\alpha x)\right] / x\right\} / c \\
& \mathcal{B}^{\varphi}=i \alpha^{-2}\left\{i m \kappa\left[C J_{m}(\alpha x)+D Y_{m}(\alpha x)\right] / x+\epsilon w \alpha\left[A J_{m}^{\prime}(\alpha x)+B Y_{m}^{\prime}(\alpha x)\right]\right\} / c \\
& \mathcal{B}^{z}=\left[C J_{m}(\alpha x)+D Y_{m}(\alpha x)\right] / c \\
& \mathcal{E}^{r}=A x^{-1}+B x \\
& \mathcal{E}^{\varphi}=i\left(C x^{-1}+D x\right) \\
& \mathcal{E}^{z}=2 i \kappa^{-1} B \\
& \mathcal{B}^{r}=-\sigma_{k} i\left(C x^{-1}+D x\right) \sqrt{\epsilon} / c \\
& \mathcal{B}^{\varphi}=\sigma_{k}\left(A x^{-1}+B x\right) \sqrt{\epsilon} / c \\
& \mathcal{B}^{z}=\sigma_{k} 2 \kappa^{-1} D \sqrt{\epsilon} / c \\
& \mathcal{E}^{r}=A x^{-2}+B x^{2}+C+D \ln (x) \\
& \mathcal{E}^{\varphi}=\sigma_{m} i\left[-A x^{-2}-B x^{2}+C+D \ln (x)\right] \\
& \mathcal{E}^{z}=i \kappa^{-1}\left(D x^{-1}+4 B x\right) \\
& \mathcal{B}^{r}=\sigma_{k} \sigma_{m} i\left[\left(A+D / \kappa^{2}\right) x^{-2}+B x^{2}-\left(C-4 B / \kappa^{2}\right)-D \ln (x)\right] \sqrt{\epsilon} / c \\
& \mathcal{B}^{\varphi}=\sigma_{k}\left[\left(A+D / \kappa^{2}\right) x^{-2}+B x^{2}+\left(C-4 B / \kappa^{2}\right)+D \ln (x)\right] \sqrt{\epsilon} / c \\
& \mathcal{B}^{z}=\sigma_{k} \sigma_{m} \kappa^{-1}\left(D x^{-1}-4 B x\right) \sqrt{\epsilon} / c \\
& \mathcal{E}^{r}=A x^{-|m|-1}+B x^{-|m|+1}+C x^{|m|-1}+D x^{|m|+1} \\
& \mathcal{E}^{\varphi}=\sigma_{m} i\left(-A x^{-|m|-1}+B x^{-|m|+1}+C x^{|m|-1}-D x^{|m|+1}\right) \\
& \mathcal{E}^{z}=-2 i \kappa^{-1}\left[(|m|-1) B x^{-|m|}-(|m|+1) D x^{|m|}\right] \\
& \mathcal{B}^{r}=\sigma_{k} \sigma_{m} i\left\{\left[A-2|m|(|m|-1) B / \kappa^{2}\right] x^{-|m|-1}-B x^{-|m|+1}\right. \\
& \left.-\left[C-2|m|(|m|+1) D / \kappa^{2}\right] x^{|m|-1}+D x^{|m|+1}\right\} \sqrt{\epsilon} / c \\
& \mathcal{B}^{\varphi}=\sigma_{k}\left\{\left[A-2|m|(|m|-1) B / \kappa^{2}\right] x^{-|m|-1}+B x^{-|m|+1}\right. \\
& \left.+\left[C-2|m|(|m|+1) D / \kappa^{2}\right] x^{|m|-1}+D x^{|m|+1}\right\} \sqrt{\epsilon} / c \\
& \mathcal{B}^{z}=-\sigma_{k} \sigma_{m} 2 \kappa^{-1}\left[(|m|-1) B x^{-|m|}+(|m|+1) D x^{|m|}\right] \sqrt{\epsilon} / c \\
& \mathcal{E}^{r}=-i \tilde{\alpha}^{-2}\left\{\kappa \tilde{\alpha}\left[A I_{m}^{\prime}(\tilde{\alpha} x)+B K_{m}^{\prime}(\tilde{\alpha} x)\right]+i m w\left[C I_{m}(\tilde{\alpha} x)+D K_{m}(\tilde{\alpha} x)\right] / x\right\} \\
& \mathcal{E}^{\varphi}=-i \tilde{\alpha}^{-2}\left\{i m \kappa\left[A I_{m}(\tilde{\alpha} x)+B K_{m}(\tilde{\alpha} x)\right] / x-w \tilde{\alpha}\left[C I_{m}^{\prime}(\tilde{\alpha} x)+D K_{m}^{\prime}(\tilde{\alpha} x)\right]\right\} \\
& \mathcal{E}^{z}=A I_{m}(\tilde{\alpha} x)+B K_{m}(\tilde{\alpha} x) \\
& \mathcal{B}^{r}=-i \tilde{a}^{-2}\left\{\kappa \tilde{\alpha}\left[C I_{m}^{\prime}(\tilde{\alpha} x)+D K_{m}^{\prime}(\tilde{\alpha} x)\right]-i m \varepsilon w\left[A I_{m}(\tilde{a} x)+B K_{m}(\tilde{\alpha} x)\right] / x\right\} / c \\
& \mathcal{B}^{\varphi}=-i \tilde{\alpha}^{-2}\left\{i m \kappa\left[C I_{m}(\tilde{\alpha} x)+D K_{m}(\tilde{\alpha} x)\right] / x+\epsilon w \tilde{\alpha}\left[A I_{m}^{\prime}(\tilde{\alpha} x)+B K_{m}^{\prime}(\tilde{\alpha} x)\right]\right\} / c \\
& \mathcal{B}^{z}=\left[C I_{m}(\tilde{\alpha} x)+D K_{m}(\tilde{\alpha} x)\right] / c
\end{aligned}
$$

$( \pm m, \pm k)$ are degenerate in frequency $\omega_{\eta}$. Let us adopt the notation that solutions inside the fiber have radial partial waves with amplitudes $A, B, C, D$ (see Table IV), and solutions outside the fiber have primed amplitudes $A^{\prime}, B^{\prime}$, $C^{\prime}, D^{\prime}$. At most, two of these eight amplitudes are not fixed by the continuity conditions and the requirement that the modal fields be bounded, corresponding to one or two independent mode families.

The eigenmodes have markedly different properties depending on how their eigenfrequency $\omega_{\eta}$ compares to the vacuum light line $\omega_{c} \equiv c|k|$ and the dielectric light line $\omega_{v} \equiv v|k|$. We distinguish three cases: Modes with frequencies above the vacuum light line are radiative modes, and modes with frequencies between the vacuum and dielectric light line are fiber-guided modes. Modes on the vacuum light line are weakly guided (they decay polynomially away from the fiber surface). On the dielectric light line and below $\omega_{v} \geq \omega_{\eta}$, no modes can exist [93].

Radiative modes are distinguished by $\omega_{\eta}>\omega_{c}$. The modal fields $\boldsymbol{e}_{\eta}, \boldsymbol{b}_{\eta}$ have radial partial waves given by case (1) in Table IV both inside and outside the fiber, with amplitudes listed in Table V. There are two independent amplitudes, which implies two mode families can be distinguished. In defining these mode families, special care has to be taken to ensure they are orthogonal according to Eq. (A4); see Ref. [93] for details. Radiative modes are not confined to the fiber but permeate all of space. In consequence, their excitation spectrum in radial direction is 
TABLE V. Radiative modes: The radial partial waves of modal fields Eq. (A9) are given by case (1) in Table IV both inside and outside the fiber, with amplitudes listed in this table. Unprimed amplitudes apply inside the fiber, primed amplitudes outside the fiber. The amplitudes $A, C \in \mathbb{C}$ are independent, and $B=D=0$. We define $N \equiv Y_{m}(\beta) J_{m}^{\prime}(\beta)-Y_{m}^{\prime}(\beta) J_{m}(\beta)$. All other quantities are defined in Table III.

\begin{tabular}{l}
\hline$\overline{A^{\prime}}=\left\{A\left[\beta \epsilon Y_{m}(\beta) J_{m}^{\prime}(\alpha) / \alpha-Y_{m}{ }^{\prime}(\beta) J_{m}(\alpha)\right]+i C m \kappa\left(\beta^{2} / \alpha^{2}-1\right) Y_{m}(\beta) J_{m}(\alpha) / \beta w\right\} / N$ \\
$B^{\prime}=\left[A\left\{J_{m}(\alpha) N-J_{m}(\beta)\left[\beta \epsilon Y_{m}(\beta) J_{m}^{\prime}(\alpha) / \alpha-Y_{m}^{\prime}(\beta) J_{m}(\alpha)\right]\right\} / Y_{m}(\beta)-i C m \kappa\left(\beta^{2} / \alpha^{2}-1\right) J_{m}(\beta) J_{m}(\alpha) / \beta w\right] / N$ \\
$C^{\prime}=\left\{-i A m \kappa\left(\beta^{2} / \alpha^{2}-1\right) Y_{m}(\beta) J_{m}(\alpha) / \beta w+C\left[\beta Y_{m}(\beta) J_{m}^{\prime}(\alpha) / \alpha-Y_{m}^{\prime}(\beta) J_{m}(\alpha)\right]\right\} / N$ \\
$D^{\prime}=\left[i A m \kappa\left(\beta^{2} / \alpha^{2}-1\right) J_{m}(\beta) J_{m}(\alpha) / \beta w+C\left\{J_{m}(\alpha) N-J_{m}(\beta)\left[\beta Y_{m}(\beta) J_{m}^{\prime}(\alpha) / \alpha-Y_{m}^{\prime}(\beta) J_{m}(\alpha)\right]\right\} / Y_{m}(\beta)\right] / N$ \\
\hline
\end{tabular}

continuous; that is, for each $(m, k)$ any eigenfrequency $\omega_{\eta}>\omega_{c}$ is admissible, and a continuous index is required to label them. A possible choice is the radial constant $a$, which is real valued and positive for radiative modes. The dispersion relation of radiative modes is hence,

$$
\omega_{\eta}=v \sqrt{k^{2}+a^{2}}
$$

and they form a continuum above the vacuum light line in the $(k, \omega)$ plane, as shown in Fig. 3 .

Guided modes are characterized by $\omega_{c}>\omega_{\eta}>\omega_{v}$. The radial partial waves of their modal fields are listed explicitly in Table VI and the corresponding frequency equations in Table VII. Guided modes can propagate inside the fiber but decay exponentially far outside the fiber. For guided modes with azimuthal order $m=0$, either the electric field or the magnetic field may be transverse, while the other acquires longitudinal components. Hence, guided modes with $m=$ 0 fall into two mode families: transverse-electric (TE) modes characterized by $b_{\eta}^{z}=0$ and transverse-magnetic (TM) modes characterized by $e_{\eta}^{z}=0$. Only a discrete set of frequencies is admissible for each $(f, m, k)$ because the fields are radially confined. These frequencies correspond to the roots of the frequency equations listed in Table VII. The frequencies $\omega_{\eta}(k)$ form discrete bands in the $(k, \omega)$ plane; see Fig. 3(a). The bands can be labeled by a band index $n \in \mathbb{N}$ starting from $n=1$ for the band of lowest frequency and increasing in frequency with $n$. The modal fields of the TE and TM modes have the following symmetries with respect to the propagation constant:

$$
\begin{array}{rlrl}
e^{r}(-k) & =-e^{r}(k), & & b^{r}(-k)=-b^{r}(k), \\
e^{\varphi}(-k)=e^{\varphi}(k), & & b^{\varphi}(-k)=b^{\varphi}(k), \\
e^{z}(-k)=e^{z}(k), & & b^{z}(-k)=b^{z}(k),
\end{array}
$$

where we drop all constant mode indices.

For higher azimuthal excitations $|m| \geq 1$, both electric and magnetic field have longitudinal components, and there is only a single hybrid mode family. The resulting bands shown in Figs. 3(b) and 3(c) derive from the frequency equation

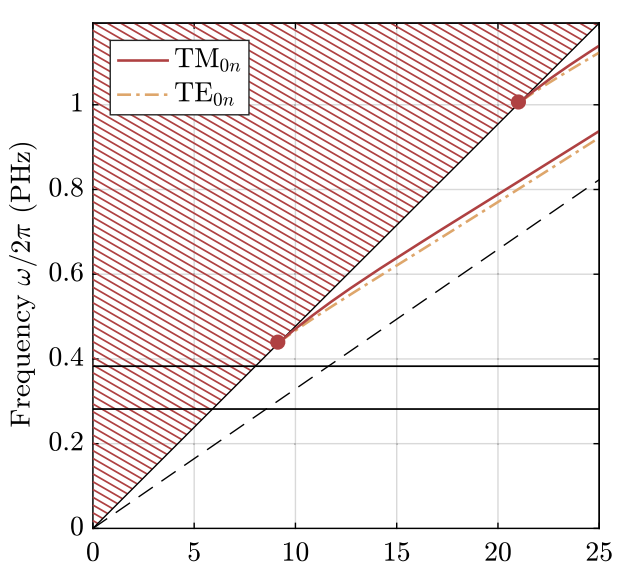

(a) $m=0$

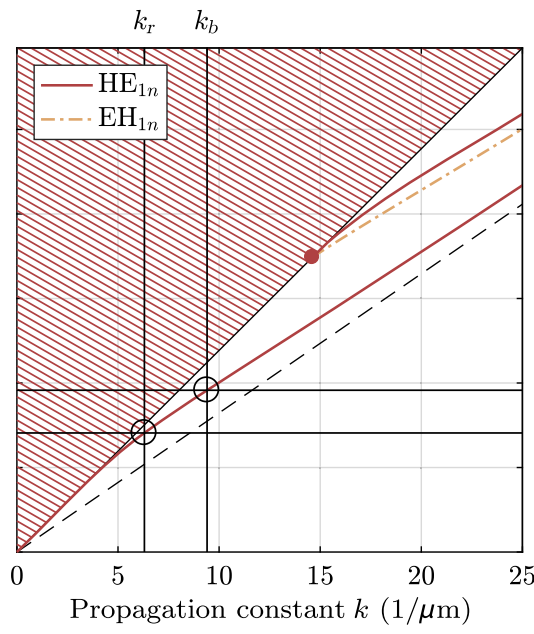

(b) $m= \pm 1$

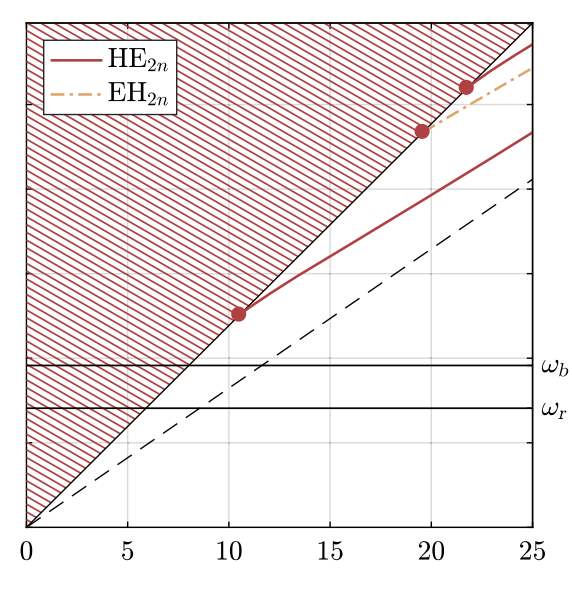

(c) $m= \pm 2$

FIG. 3. Band structures of nanofiber photon modes with azimuthal order $m=0$ in panel (a), $m= \pm 1$ in panel (b), and $m= \pm 2$ in panel (c). The radius $R$ and the relative permittivity $\epsilon$ are specified in Appendix E. The hatched area corresponds to radiative modes delimited by the vacuum light line. The dashed line delineates the dielectric light line. The cutoff frequencies, where guided bands cross over into the radiative continuum, are indicated by solid points. The modes populated in the case study in Sec. III are indicated by circles: Their frequencies $\omega_{r}, \omega_{b}$ lie below all cutoff frequencies, so only modes on the fundamental $\mathrm{HE}_{11}$ band can be populated. 
TABLE VI. Guided modes: Radial partial waves of the modal fields Eq. (A9) both inside the fiber and in the vacuum surrounding the fiber. The quantities $A, B \in \mathbb{C}$ are amplitudes. The $\mathrm{TE}_{0 n}$ modes are characterized by $A=0$, and the $\mathrm{TM}_{0 n} \operatorname{modes}$ by $B=0$. The $\mathrm{HE}_{|m| n}$ and $\mathrm{EH}_{|m| n}$ modes have the same modal fields but distinct frequencies; see Table VII. The remaining amplitude is determined from the normalization condition Eq. (A13). All other quantities are defined in Table III.

\begin{tabular}{lll}
\hline \hline Band & \multicolumn{1}{c}{ Fiber $(x<1)$} & \multicolumn{1}{c}{ Vacuum $(x>1)$} \\
\hline $\mathrm{TE}_{0 n}, \mathrm{TM}_{0 n}$ & $\mathcal{E}_{\eta}^{r}=-i \kappa \alpha^{-1} B J_{1}(\alpha x)$ & $\mathcal{E}_{\eta}^{r}=i \eta \tilde{\beta}^{-1} B K_{1}(\tilde{\beta} x)$ \\
& $\mathcal{E}_{\eta}^{\varphi}=-w \alpha^{-1} A J_{1}(\alpha x)$ & $\mathcal{E}_{\eta}^{\varphi}=\eta w \tilde{\beta}^{-1} A K_{1}(\tilde{\beta} x)$ \\
& $\mathcal{E}_{\eta}^{z}=B J_{0}(\alpha x)$ & $\mathcal{E}_{\eta}^{z}=\eta B K_{0}(\tilde{\beta} x)$ \\
& $\mathcal{B}_{\eta}^{r}=\kappa \alpha^{-1} A J_{1}(\alpha x) / c$ & $\mathcal{B}_{\eta}^{r}=-\eta \kappa \tilde{\beta}^{-1} A K_{1}(\tilde{\beta} x) / c$ \\
& $\mathcal{B}_{\eta}^{\varphi}=-i \epsilon w \alpha^{-1} B J_{1}(\alpha x) / c$ & $\mathcal{B}_{\eta}^{\varphi}=i \eta w \tilde{\beta}^{-1} B K_{1}(\tilde{\beta} x) / c$ \\
& $\mathcal{B}_{\eta}^{z}=i A J_{0}(\alpha x) / c$ & $\mathcal{B}_{\eta}^{z}=i \eta A K_{0}(\tilde{\beta} x) / c$ \\
$\mathrm{HE}_{|m| n}, \mathrm{EH}_{|m| n}$ & $\mathcal{E}_{\eta}^{r}=i \alpha^{-2} A\left[\kappa \alpha J_{m}^{\prime}(\alpha x)-m^{2} w \gamma J_{m}(\alpha x) / x\right]$ & $\mathcal{E}_{\eta}^{r}=-i \eta \tilde{\beta}^{-2} A\left[\kappa \tilde{\beta} K_{m}^{\prime}(\tilde{\beta} x)-m^{2} w \gamma K_{m}(\tilde{\beta} x) / x\right]$ \\
& $\mathcal{E}_{\eta}^{\varphi}=m \alpha^{-2} A\left[w \gamma \alpha J_{m}^{\prime}(\alpha x)-\kappa J_{m}(\alpha x) / x\right]$ & $\mathcal{E}_{\eta}^{\varphi}=-m \eta \tilde{\beta}^{-2} A\left[w \gamma \tilde{\beta} K_{m}^{\prime}(\tilde{\beta} x)-\kappa K_{m}(\tilde{\beta} x) / x\right]$ \\
& $\mathcal{E}_{\eta}^{z}=A J_{m}(\alpha x)$ & $\mathcal{E}_{\eta}^{z}=\eta A K_{m}(\tilde{\beta} x)$ \\
& $\mathcal{B}_{\eta}^{r}=-m \alpha^{-2} A\left[\kappa \gamma \alpha J_{m}^{\prime}(\alpha x)-\varepsilon w J_{m}(\alpha x) / x\right] / c$ & $\left.\mathcal{B}_{\eta}^{r}=m \eta \tilde{\beta}\right)^{-2} A\left[\kappa \gamma \tilde{\beta} K_{m}^{\prime}(\tilde{\beta} x)-w K_{m}(\tilde{\beta} x) / x\right] / c$ \\
& $\mathcal{B}_{\eta}^{\varphi}=i \alpha^{-2} A\left[\varepsilon w \alpha J_{m}^{\prime}(\alpha x)-m^{2} \kappa \gamma J_{m}(\alpha x) / x\right] / c$ & $\mathcal{B}_{\eta}^{\varphi}=-i \eta \tilde{\beta}^{-2} A\left[w \tilde{\beta} K_{m}^{\prime}(\tilde{\beta} x)-m^{2} \kappa \gamma K_{m}(\tilde{\beta} x) / x\right] / c$ \\
\hline \hline
\end{tabular}

$$
\begin{aligned}
& {\left[\alpha J_{m}(\alpha) K_{m}^{\prime}(\tilde{\beta})+\tilde{\beta} K_{m}(\tilde{\beta}) J_{m}^{\prime}(\alpha)\right]} \\
& \times\left[\alpha J_{m}(\alpha) K_{m}^{\prime}(\tilde{\beta})+\epsilon \tilde{\beta} K_{m}(\tilde{\beta}) J_{m}^{\prime}(\alpha)\right] \\
& \quad=\left[m \frac{\kappa w}{\alpha \tilde{\beta}}(\epsilon-1) J_{m}(\alpha) K_{m}(\tilde{\beta})\right]^{2},
\end{aligned}
$$

with parameters defined in Table III. There are, however, two subclasses of modes called $\mathrm{HE}$ and $\mathrm{EH}$ distinguished by the asymptotes of their bands as $|k| \rightarrow \infty$. Each band of HE (EH) modes asymptotically approaches a root of the Bessel function of the first kind $J_{m-1}(a R)\left(J_{m+1}(a R)\right)$, and the electric (magnetic) field has a longitudinal component of significant magnitude compared to its transverse components $[87,89]$. As the propagation constant $k$ is the only continuous index for guided modes, the orthonormality condition Eq. (A4) in conjunction with Eq. (A6) reduces to a normalization condition for the electric radial partial waves:

$$
\int_{0}^{\infty} r\left|\mathcal{E}_{\eta}(r)\right|^{2} \epsilon(r) d r=\frac{1}{\epsilon_{0}^{2}} .
$$

The modal fields of the $\mathrm{HE}$ and $\mathrm{EH}$ modes have the following symmetries with respect to the azimuthal order $m$ and propagation constant $k$ :

$$
\begin{aligned}
& e_{\sigma m}^{r}\left(\sigma^{\prime} k\right)=\sigma^{m} \sigma^{\prime} e_{m}^{r}(k), \quad b_{\sigma m}^{r}\left(\sigma^{\prime} k\right)=\sigma^{m+1} b_{m}^{r}(k), \\
& e_{\sigma m}^{\varphi}\left(\sigma^{\prime} k\right)=\sigma^{m+1} \sigma^{\prime} e_{m}^{\varphi}(k), \quad b_{\sigma m}^{\varphi}\left(\sigma^{\prime} k\right)=\sigma^{m} b_{m}^{\varphi}(k), \\
& e_{\sigma m}^{z}\left(\sigma^{\prime} k\right)=\sigma^{m} e_{m}^{z}(k), \quad b_{\sigma m}^{z}\left(\sigma^{\prime} k\right)=\sigma^{m+1} \sigma^{\prime} b_{m}^{z}(k),
\end{aligned}
$$

where $\sigma, \sigma^{\prime}= \pm 1$.

Weakly guided modes with frequencies on the vacuum light line $\omega_{c}=\omega_{\eta}$ appear where bands of guided modes cross over into the radiative continuum. Inside the fiber, the radial partial waves of the modal fields are given by case (1) listed in Table IV (since $\omega_{\eta}>\omega_{v}$ ). Outside the fiber, they are given by case (2) with $\epsilon=1$, and therefore, replacing $\alpha$ with $\beta$; see Table III. The corresponding amplitudes are listed in Table VIII. The modal fields decay polynomially outside the fiber as a limiting case between oscillatory behavior and exponential decay. The frequency equations on the vacuum light line listed in Table VII mark the cutoff frequencies below which a band of guided modes ceases to exist. The cutoff frequencies are indicated by solid dots in Fig. 3.

In summary, guided modes can be labeled by mode indices

TABLE VII. Guided modes: Frequency equations and cutoff frequencies in terms of the quantities defined in Table III. The roots of these equation form discrete bands in the $(k, \omega)$ plane as shown in Fig. 3.

\begin{tabular}{lcc}
\hline \hline Band & Frequency equation & Cutoff frequency \\
\hline $\mathrm{TE}_{0 n}$ & $\alpha J_{0}(\alpha) K_{1}(\tilde{\beta})+\tilde{\beta} K_{0}(\tilde{\beta}) J_{1}(\alpha)=0$ & $J_{0}(\alpha)=0$ \\
$\mathrm{TM}_{0 n}$ & $\alpha J_{0}(\alpha) K_{1}(\tilde{\beta})+\varepsilon \tilde{\beta} K_{0}(\tilde{\beta}) J_{1}(\alpha)=0$ & $J_{0}(\alpha)=0$ \\
$\mathrm{HE}_{|m| n}$ & Odd roots of Eq. (A12) & {$\left[|m|(|m|-1)-\alpha^{2} /(\varepsilon+1)\right] J_{m}(\alpha)+(|m|-1) \alpha J_{m}{ }^{\prime}(\alpha)=0$} \\
$\mathrm{EH}_{|m| n}$ & Even roots of Eq. (A12) & $J_{m}(\alpha)=0$ \\
\hline \hline
\end{tabular}


TABLE VIII. Weakly guided modes: The modal fields Eq. (A9) have radial partial waves given by case (1) in Table IV inside the fiber and by case (2) outside the fiber, with nonzero amplitudes listed in this table. Unprimed amplitudes apply inside the fiber, primed amplitudes outside the fiber. Amplitudes that are not listed vanish. All other quantities are defined in Table III.

\begin{tabular}{|c|c|}
\hline Band & Amplitudes \\
\hline $\begin{array}{l}\mathrm{TE}_{0 n} \\
\mathrm{TM}_{0 n}\end{array}$ & $\begin{array}{l}C \in \mathbb{C} \\
C^{\prime}=\sigma_{k} C \kappa \alpha^{-1} J_{1}(\alpha) \\
A \in \mathbb{C} \\
A^{\prime}=-i A \epsilon \kappa \alpha^{-1} J_{1}(\alpha)\end{array}$ \\
\hline Band & Amplitudes \\
\hline $\mathrm{HE}_{1 n}$ & $\begin{array}{l}C \in \mathbb{C} \\
A^{\prime}=\sigma_{k} C m \kappa \alpha^{-1} J_{m}^{\prime}(\alpha) / 2 \\
C^{\prime}=-\sigma_{k} C m \kappa \alpha^{-1} J_{m}^{\prime}(\alpha) / 2 \\
A \in \mathbb{C} \\
A^{\prime}=i A \epsilon \kappa \alpha^{-2} J_{m}^{\prime}(\alpha) / 2 \\
C^{\prime}=i A \epsilon \kappa \alpha^{-2} J_{m}^{\prime}(\alpha) / 2\end{array}$ \\
\hline Band & Amplitudes \\
\hline $\begin{array}{l}\mathrm{HE}_{|m| n} \\
|m| \geq 2\end{array}$ & $\begin{array}{l}A \in \mathbb{C} \\
C=-i A \sigma_{m} \sigma_{k} \\
A^{\prime}=i A(\epsilon-1) \kappa J_{m}(\alpha) / 2(\epsilon+1)(|m|-1) \\
B^{\prime}=i A \kappa J_{m}(\alpha) / 2(|m|-1) \\
C \in \mathbb{C} \\
A=-i \sigma_{m} \sigma_{k} C / \varepsilon \\
A^{\prime}=\sigma_{m} \sigma_{p} C \kappa \alpha^{-1} J_{m}^{\prime}(\alpha)\end{array}$ \\
\hline
\end{tabular}

$$
\begin{array}{rlrl}
m \in \mathbb{Z}, & f \in\{\mathrm{TE}, \mathrm{TM}\}_{m=0} & \text { or } & \{\mathrm{HE}, \mathrm{EH}\}_{m \neq 0}, \\
k \in \mathbb{R}, & n \in \mathbb{N} .
\end{array}
$$

It is customary to name guided bands as $f_{|m| n}$. At $m=0$, there are then $\mathrm{TE}_{0 n}$ and $\mathrm{TM}_{0 n}$ bands, and at $|m| \geq 1$ there are $\mathrm{HE}_{|m| n}$ and $\mathrm{EH}_{|m| n}$ bands. For a given azimuthal order $m$, the $\mathrm{HE}$ and $\mathrm{EH}$ bands alternate, with the $\mathrm{HE}_{|m| 1}$ band of lowest frequencies; see Figs. 3(b) and 3(c). At sufficiently low frequencies, only the $\mathrm{HE}_{11}$ band is guided, while all other bands merge into the radiative continuum (singlemode regime); compare Fig. 3(b) to Figs. 3(a) and 3(c). These low-frequency $\mathrm{HE}_{11}$ modes are used as trapping fields in nanofiber-based cold-atom traps.

\section{APPENDIX B: ATOM TRAP AND MOTIONAL STATES}

In the first part of this Appendix, we summarize how to obtain the optical and surface potentials experienced by an atom trapped close to a photonic structure. In the second part, we discuss under which conditions the potential can be approximated as harmonic in the case of a nanofiberbased atom trap.

\section{Trapping potential}

The internal and external dynamics of an atom trapped close to a photonic structure is governed by the Hamiltonian

$$
\hat{H}_{\mathrm{at}}=\hat{H}_{\mathrm{int}}+\frac{\hat{\boldsymbol{p}}^{2}}{2 M}+\hat{V}_{\mathrm{opt}}+\hat{V}_{\mathrm{ad}}
$$

in the absence of vibrations of the structure. Here, $\hat{H}_{\text {int }}$ describes the internal state of the atom, $\hat{\boldsymbol{p}}$ is the momentum operator of its center of mass, and the remaining terms are introduced in Sec. II. The internal hyperfine-structure states of the atom can be labeled by the eigenvalues of a suitable set of commuting operators, for instance, $|\lambda\rangle \equiv|n S L J I F\rangle$, where $n$ is the principal quantum number, $S$ is the electron spin, $L$ is the electron orbital angular momentum, $J$ is the total electronic angular momentum, $I$ the nuclear spin, and $F$ the resulting total atom angular momentum [94]. The optical trapping fields are detuned from resonance with the atom, such that they do not excite the atom from the electronic ground state. Instead, both light and surface effects lead to a slight mixing (dressing) of the internal eigenstates $|\lambda\rangle$ of the atom [70]. The new, dressed eigenstates have energies shifted by an amount typically much smaller than the splitting between hyperfine-structure levels of different $F$. The dressed eigenstates are therefore very similar to the bare eigenstates $|\lambda\rangle$ and can be labeled using the same quantum numbers. Gradients in the light intensity then lead to position-dependent light shifts, which act as an optical potential for the center of mass and thus allow trapping of the atom [44]. Both optical and surface potentials depend on the internal state $|\lambda\rangle$ of the atom because the electric polarizability of the atom is state dependent [56,94]. Moreover, the atom-light interaction can couple internal and motional states [24,28].

We focus on scenarios without coupling of internal and motional states, such that the potential operators $\hat{V}_{\text {opt }}$ and $\hat{V}_{\text {ad }}$ are block diagonal in the dressed hyperfine-structure levels: $\hat{V}_{\text {opt }}+\hat{V}_{\text {ad }}=\sum_{\lambda}\left[V_{\text {opt }, \lambda}(\hat{\boldsymbol{r}})+V_{\text {ad }, \lambda}(\hat{\boldsymbol{r}})\right]|\lambda\rangle\langle\lambda|$. The motion of the atom in the trap is thus governed by the Hamiltonian

$$
\hat{H}_{\mathrm{at}}=\frac{\hat{\boldsymbol{p}}^{2}}{2 M}+V_{0}(\hat{\boldsymbol{r}})
$$

in each subspace of the Hilbert space with fixed internal state $|\lambda\rangle$. Energies are measured relative to the energy $\left\langle\lambda\left|\hat{H}_{\text {int }}\right| \lambda\right\rangle$ of the internal state, and $V_{0} \equiv V_{\mathrm{opt}, \lambda}+V_{\mathrm{ad}, \lambda}$ is the total statedependent potential. In two-color traps in particular, two monochromatic light fields are used, tuned in opposite directions away from the resonance frequency of the atom [49]. The red-detuned field attracts the atom toward the surface of the nanophotonic structure. The repulsive bluedetuned field has a shorter decay length and dominates closer to the surface, keeping the atom at a distance. If the two light fields are sufficiently far detuned, interference between them is negligible, and the optical potential is the sum of the 


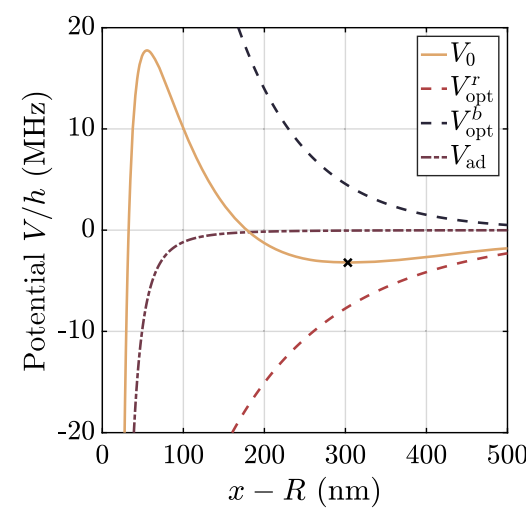

(a) Contributions to potential at $z=z_{0}, y=y_{0}=0$.

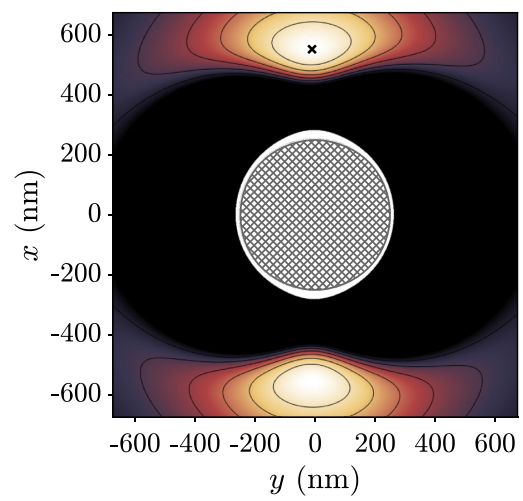

(b) Cross section at $z=z_{0}$.

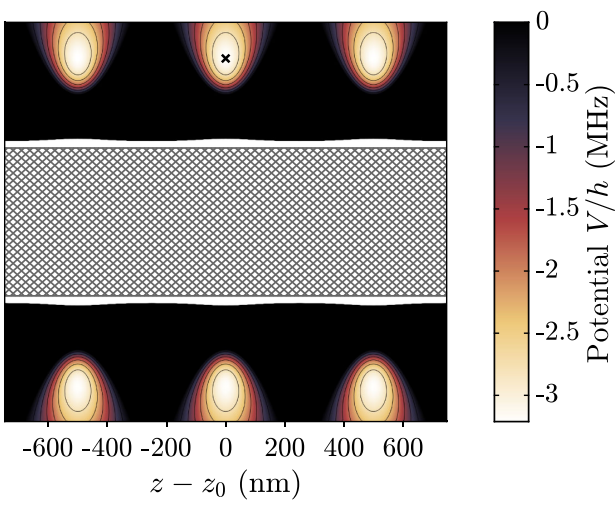

(c) Longitudinal section at $y=y_{0}=0$.

FIG. 4. Total potential experienced by the atom, with a trap minimum $\boldsymbol{r}_{0}$ indicated by the cross. Panel (a) shows the different contributions to the total potential $V_{0}$. Panels (b) and (c) show the total potential in a cross section and longitudinal section of the fiber, respectively. All positive values of the potential in (b) and (c) are shown as black. The parameters used for this plot are listed in Appendix E.

individual contributions $V_{\mathrm{opt}}^{r}$ and $V_{\mathrm{opt}}^{b}$ of the red- and bluedetuned field, respectively. The total state-dependent potential is then

$$
V_{0} \equiv V_{\mathrm{opt}}^{r}+V_{\mathrm{opt}}^{b}+V_{\mathrm{ad}},
$$

where we drop the index $\lambda$ from notation. Figure 4 shows an example of how these three contributions combine to a threedimensional trapping potential close to an optical nanofiber.

The optical potential $V_{\text {opt }}$ created by each monochromatic light field $\boldsymbol{E}(\boldsymbol{r}, t)=\boldsymbol{E}_{0}(\boldsymbol{r}) e^{-i \omega t}+$ c.c. of frequency $\omega$ can be expressed in terms of a scalar, vector, and tensor light shift [94],

$$
V_{\mathrm{opt}}=V_{s}+V_{v}+V_{t} \text {. }
$$

We assume that there is a homogeneous magnetic offset field $\boldsymbol{B}_{\text {ext }}=B_{\text {ext }} z_{B}$ applied along the unit vector $z_{B}$, which induces Zeeman splitting of the hyperfine structure. The internal dressed eigenstates of the atom are then the Zeeman substates $|\lambda\rangle=\left|\xi F M_{F}\right\rangle$, provided the magnetic field is sufficiently strong to avoid mixing of the Zeeman substates by their relative light shifts. Here, $|\xi\rangle=|n S L J I\rangle$ is the fine-structure state, and $M_{F}$ is the magnetic quantum number of the total atom angular momentum with respect to the quantization axis $z_{B}$. The scalar light shift is

$$
V_{s}(\boldsymbol{r})=-\alpha_{s}\left|\boldsymbol{E}_{0}(\boldsymbol{r})\right|^{2},
$$

with a scalar polarizability $\alpha_{s}$ that depends only on its finestructure state $|\xi\rangle$. The vector light shift is [94]

$$
V_{v}(\boldsymbol{r})=-\frac{\alpha_{v}}{2 i} \frac{M_{F}}{F}\left[\boldsymbol{E}_{0}^{*}(\boldsymbol{r}) \times \boldsymbol{E}_{0}(\boldsymbol{r})\right] \cdot z_{B} .
$$

The vector polarizability $\alpha_{v}$ of the hyperfine-structure Zeeman substate $|\lambda\rangle$ can be obtained from the vector polarizability $\tilde{\alpha}_{v}$ of the fine-structure state $|\xi\rangle$ :

$$
\alpha_{v}=\frac{F(F+1)+J(J+1)-I(I+1)}{(F+1) 2 J} \tilde{\alpha}_{v} .
$$

The tensor light shift is

$$
V_{t}(\boldsymbol{r})=-3 \alpha_{t} \frac{3 M_{F}^{2}-F(F+1)}{2 F(2 F-1)}\left[\left|E_{0}^{z_{B}}(\boldsymbol{r})\right|^{2}-\frac{1}{3}\right],
$$

where $E_{0}^{z_{B}}=\boldsymbol{E}_{0} \cdot z_{B}$, and the hyperfine-structure tensor polarizability $\alpha_{t}$ is related to the fine-structure tensor polarizability $\tilde{\alpha}_{t}$ by

$$
\begin{aligned}
& \alpha_{t}=(-1)^{(J+I+F)} \sqrt{\frac{3(J+1)(2 J+1)(2 J+3)}{2 J(2 J-1)}} \\
& \times \sqrt{\frac{2 F(2 F-1)(2 F+1)}{3(F+1)(2 F+3)}}\left\{\begin{array}{lll}
F & 2 & F \\
J & I & J
\end{array}\right\} \tilde{\alpha}_{t} .
\end{aligned}
$$

Here, $\{\cdot\}$ is the Wigner $6 j$ symbol. The fine-structure polarizabilities $\tilde{\alpha}_{s}=\alpha_{s}, \tilde{\alpha}_{v}$, and $\tilde{\alpha}_{t}$ can be calculated from experimental data [94].

At atom-surface separations realized in nanophotonic traps, surface effects are limited to dispersion forces [95]. The dispersion force between solids and atoms strongly depends on both geometry and material. Its effect can be modeled by the nonretarded Casimir-Polder potential for a two-level atom in the ground state located in the vicinity of a dielectric object [56]. In the case of a nanofiberbased trap, it is sufficient to model the fiber as a dielectric half-space [49,77], although the potential for an atom close to a dielectric cylinder can in principle be calculated analytically $[49,98-100]$. The potential is then 


$$
V_{\mathrm{ad}}(r)=-C(r-R)^{-3}
$$

The parameter $C>0$ can either be obtained experimentally [101] or calculated from the electromagnetic properties of the material $[56,98,102,103]$.

\section{Harmonic trap approximation}

A quadratic atom-phonon interaction Hamiltonian [104] can be obtained by approximating the trapping potential as harmonic for atoms close in energy to the motional ground state. In the case of a nanofiber-trapped atom in particular, we choose cylindrical coordinates to describe the motion of the trapped atom. The Hamiltonian $\hat{H}_{\text {at }}$ describing the motion of the atom in the trap in the absence of vibrations is then

$$
\hat{H}_{\mathrm{at}}=\frac{\left(\hat{p}^{r}\right)^{2}}{2 M}-\frac{\hbar^{2}}{8 M \hat{r}^{2}}+\frac{\left(\hat{p}^{\varphi}\right)^{2}}{2 M \hat{r}^{2}}+\frac{\left(\hat{p}^{z}\right)^{2}}{2 M}+V_{0}(\hat{\boldsymbol{r}}),
$$

where $\hat{\boldsymbol{r}}=(\hat{\boldsymbol{r}}, \hat{\varphi}, \hat{z})$ is the position operator of the atom, and $\left(\hat{p}^{r}, \hat{p}^{\varphi}, \hat{p}^{z}\right)$ are the components of the momentum operator. We expand the potential to second order around the local trap minimum $\boldsymbol{r}_{0}$. The corresponding motional frequencies of the atom with respect to the coordinates $\left(x^{r}, x^{\varphi}, x^{z}\right) \equiv$ $\left(r, r_{0} \varphi, z\right)$ are

$$
\omega_{i} \equiv \sqrt{\frac{\partial_{x^{i}}^{2} V\left(\boldsymbol{r}_{0}\right)}{M}}, \quad \omega_{i j} \equiv \sqrt{\frac{\partial_{x^{i}} \partial_{x^{j}} V\left(\boldsymbol{r}_{0}\right)}{M}}
$$

where $i, j \in\{r, \varphi, z\}$. The cross-derivatives of the potential may be nonzero, since the symmetry axes of the potential are in general not aligned with the coordinate axes (for instance, when the magnetic offset field breaks the cylindrical symmetry of the setup). The Hamiltonian Eq. (B11) then describes harmonic motion of the atom in each direction around the trap minimum, provided $\omega_{i j}=0$ and given that the trap is far from the fiber axis compared to its size: $\omega_{r} \gg \hbar /\left(2 M r_{0}^{2}\right)$ and $\omega_{\varphi} \gg \hbar /\left(4 \pi M r_{0}^{2}\right)$. Introducing ladder operators $\hat{a}_{i}$ and $\hat{a}_{i}^{\dagger}$, the position operators $\hat{x}^{i}$ can then be expressed as

$$
\hat{x}^{i}=\Delta x^{i}\left(\hat{a}_{i}+\hat{a}_{i}^{\dagger}\right)+x_{0}^{i},
$$

where $x_{0}^{i}$ is the position of the trap minimum, and $\left(\Delta x^{r}, \Delta x^{\varphi}, \Delta x^{z}\right) \equiv\left(\Delta r, r_{0} \Delta \varphi, \Delta z\right) \quad$ is the zero-point motion of the atom in the trap as defined in Sec. II. If $\omega_{i j} \neq 0$, there is additional cross-coupling between the motional modes of the atom, with coupling constants $g_{i j}=\omega_{i j}^{2} / 4 \sqrt{\omega_{i} \omega_{j}}$. The atom Hamiltonian is then in general
$\hat{H}_{\mathrm{at}}=\sum_{i} \hbar \omega_{i}\left(\hat{a}_{i}^{\dagger} \hat{a}_{i}+\frac{1}{2}\right)+\sum_{i, j \neq i} \hbar g_{i j}\left(\hat{a}_{i}+\hat{a}_{i}^{\dagger}\right)\left(\hat{a}_{j}+\hat{a}_{j}^{\dagger}\right)$,

where we measure energies relative to the depth of the trapping potential $V_{0}=V\left(\boldsymbol{r}_{0}\right)$. The Hamiltonian can always be diagonalized [105], and hence simplifies to Eq. (2) after dropping the constant zero-point energy.

\section{APPENDIX C: PHONONIC EIGENMODES}

Vibrations of the photonic structure in a nanophotonic cold-atom trap alter the optical fields surrounding the structure. This variation leads to an interaction of the vibrations and the trapped atoms, as we discuss in detail in Appendix D. Vibrations at frequencies relevant to nanophotonic traps can be modeled by linear elasticity theory, because the corresponding phonon wavelengths are sufficiently large not to resolve the microscopic structure of the solid. Linear elasticity theory describes the dynamics of elastic deformations of a continuous body around its equilibrium state [58-60]. The deformations are described by the displacement field $\boldsymbol{u}$, a real-valued vector field defined on the domain of the body. The displacement field indicates the magnitude and direction of the displacement of each point of the body from equilibrium at any given time.

Our objective is to provide a quantum description of the vibrations (in terms of a phonon field) and of the atomphonon interaction. In this Appendix, we review how a quantum formulation of linear elasticity can be obtained through canonical quantization based on the concept of phononic eigenmodes. Subsequently, we discuss the phononic eigenmodes of a nanofiber.

\section{Quantum elastodynamics}

Consider an elastic body in three dimensions. Within the framework of linear elasticity, its mechanical properties are described by the mass density $\rho$ and the elasticity tensor $\boldsymbol{C}$, both of which are in general position dependent. The elasticity tensor is of fourth order, with symmetries $C^{i j k l}=$ $C^{j i k l}=C^{i j l k}=C^{k l i j}[60]$. In the case of a homogeneous elastic body, $\rho$ and $\boldsymbol{C}$ are constant. If the body is isotropic, the elasticity tensor has the form $[58,60]$

$$
C^{i j k l}=\mu\left[\delta^{i k} \delta^{j l}+\delta^{i l} \delta^{j k}\right]+\lambda \delta^{i j} \delta^{k l} .
$$

The two coefficients $\lambda$ and $\mu$ are called Lamé parameters. The mechanical properties of a homogeneous and isotropic body are thus described by three real numbers: $\rho, \lambda$, and $\mu$. The density $\rho$ and Lamé's second parameter $\mu$ are positive, while Lamé's first parameter $\lambda$ may be negative [60]. An alternative, widespread parametrization uses Young's modulus $E$ and the Poisson ratio $\nu$ : 


$$
\lambda=\frac{\nu E}{(1+\nu)(1-2 \nu)}, \quad \mu=\frac{E}{2(1+\nu)} .
$$

The modulus is positive, as is the Poisson ratio for most materials [60].

The dynamics of the displacement field is governed by the equation of motion [58-60]

$$
\rho \ddot{\boldsymbol{u}}=\mathcal{D} \boldsymbol{u},
$$

where we define the differential operator $\mathcal{D}$ that acts on a vector field as $[\mathcal{D} \boldsymbol{u}]^{i} \equiv \sum_{j k l} \partial_{j} C^{i j k l} \partial_{k} u^{l}$. It is common to introduce the strain tensor $\boldsymbol{S}$ describing deformations of the solid and the stress tensor $\boldsymbol{T}$, which characterizes the forces needed to affect this strain:

$$
\begin{aligned}
S^{i j} & \equiv \frac{1}{2}\left(\partial_{i} u^{j}+\partial_{j} u^{i}\right), \\
T^{i j} & \equiv \sum_{k l} C^{i j k l} S^{k l} .
\end{aligned}
$$

Note that both strain and stress tensor are symmetric, $S^{i j}=$ $S^{j i}$ and $T^{i j}=T^{j i}$. It is necessary to specify boundary conditions in order to obtain a unique solution given initial conditions [60]. For a body that is not subject to external forces, these boundary conditions are of Neumann type and state that on the surface of the body, the stress vanishes in direction $\boldsymbol{n}$ normal to the surface: $\boldsymbol{T n}=\mathbf{0}$.

We are interested in quantizing the vibrations of a forcefree, homogeneous, and isotropic elastic body. We start from the Lagrange density $\mathcal{L}=\rho \dot{\boldsymbol{u}}^{2} / 2-\sum_{i j} S^{i j} T^{i j} / 2$, which yields the correct equations of motion. The canonical conjugate momentum is then $\boldsymbol{\pi}=\rho \dot{\boldsymbol{u}}$, and the resulting classical Hamilton functional can be expressed as

$$
H=\frac{1}{2} \int_{B}\left[\frac{\pi^{2}}{\rho}-\boldsymbol{u} \cdot \mathcal{D} \boldsymbol{u}\right] d \boldsymbol{r},
$$

where $B$ is the volume of the body [106].

To proceed, we introduce phononic eigenmodes $\boldsymbol{w}_{\gamma}$ with eigenfrequencies $\omega_{\gamma}$ as solutions of the eigenvalue equation

$$
\mathcal{D} \boldsymbol{w}_{\gamma}(\boldsymbol{r})=-\rho \omega_{\gamma}^{2} \boldsymbol{w}_{\gamma}(\boldsymbol{r})
$$

together with the boundary conditions for a force-free body. The eigenmodes are labeled using a multi-index $\gamma$ which may contain both discrete and continuous indices. Since $(-\mathcal{D})$ is self-adjoint [107], eigenmode solutions form an orthogonal basis for the space of admissible displacement fields and can be normalized according to

$$
\int_{B} \boldsymbol{w}_{\gamma}^{*}(\boldsymbol{r}) \cdot \boldsymbol{w}_{\gamma^{\prime}}(\boldsymbol{r}) d \boldsymbol{r}=\delta_{\gamma \gamma^{\prime}}
$$

Any solution to the equation of motion (C3) can then be expressed as a linear combination of eigenmodes,

$$
\boldsymbol{u}(\boldsymbol{r}, t)=\sum_{\gamma} \frac{1}{\rho \omega_{\gamma}}\left[\beta_{\gamma} e^{-i \omega_{\gamma} t} \boldsymbol{w}_{\gamma}(\boldsymbol{r})+\text { c.c. }\right],
$$

with coefficients $\beta_{\gamma} \in \mathbb{C}$ determined by the initial conditions.

Canonical quantization amounts to turning every eigenmode into a bosonic mode with ladder operators $\hat{b}_{\gamma}$ and $\hat{b}_{\gamma}^{\dagger}$ that satisfy canonical commutation relations, $\left[\hat{b}_{\gamma}, \hat{b}_{\gamma^{\prime}}^{\dagger}\right]=\delta_{\gamma \gamma^{\prime}}$. The displacement field and its conjugate momentum are promoted to field operators with mode expansions

$$
\begin{aligned}
& \hat{\boldsymbol{u}}(\boldsymbol{r})=\sum_{\gamma} \mathcal{U}_{\gamma}\left[\hat{b}_{\gamma} \boldsymbol{w}_{\gamma}(\boldsymbol{r})+\text { H.c. }\right], \\
& \hat{\boldsymbol{\pi}}(\boldsymbol{r})=-i \sum_{\gamma} \Pi_{\gamma}\left[\hat{b}_{\gamma} \boldsymbol{w}_{\gamma}(\boldsymbol{r})-\text { H.c. }\right] .
\end{aligned}
$$

Here, $\mathcal{U}_{\gamma} \equiv \sqrt{\hbar / 2 \rho \omega_{\gamma}}$ and $\Pi_{\gamma} \equiv \sqrt{\hbar \rho \omega_{\gamma} / 2}$ are the mode densities. The Hamiltonian takes the form

$$
\hat{H}_{\mathrm{phn}}=\sum_{\gamma} \hbar \omega_{\gamma} \hat{b}_{\gamma}^{\dagger} \hat{b}_{\gamma}
$$

where we set the energy of the ground state to zero. Since strain plays an important role in the atom-phonon interaction discussed in Appendix D, we introduce the tensorial strain modal fields $\boldsymbol{s}_{\gamma}$ with components

$$
s_{\gamma}^{i j} \equiv \frac{1}{2}\left[\partial_{i} w_{\gamma}^{j}+\partial_{j} w_{\gamma}^{i}\right]
$$

such that the strain operator can be expressed as

$$
\hat{\boldsymbol{S}}(\boldsymbol{r})=\sum_{\gamma} \mathcal{U}_{\gamma}\left[\hat{b}_{\gamma} \boldsymbol{s}_{\gamma}(\boldsymbol{r})+\text { H.c. }\right] \text {. }
$$

\section{Phononic fiber eigenmodes}

We now consider vibrations of a nanofiber modeled as a homogeneous and isotropic cylinder of radius $R$ and of infinite length along the $z$ axis. In the following, we summarize the resulting phononic eigenmodes and frequency equations. Details on the derivation can be found in Refs. [58,59,108,109].

Solving the eigenvalue equation (C6) in cylindrical coordinates leads to phononic eigenmodes and corresponding strain modal fields of the form

$$
\begin{aligned}
& \boldsymbol{w}_{\gamma}(\boldsymbol{r})=\frac{\mathcal{W}_{\gamma}(r)}{2 \pi} e^{i(j \varphi+p z)}, \\
& \boldsymbol{s}_{\gamma}(\boldsymbol{r})=\frac{\mathcal{S}_{\gamma}(r)}{2 \pi} e^{i(j \varphi+p z)},
\end{aligned}
$$

in close analogy to the photon modes Eq. (A9). Here, $p \in \mathbb{R}$ is the propagation constant and $j \in \mathbb{Z}$ the azimuthal order. Mode quadruplets $( \pm j, \pm p)$ are degenerate in eigenfrequency $\omega_{\gamma}$. Since phonon modes are radially 


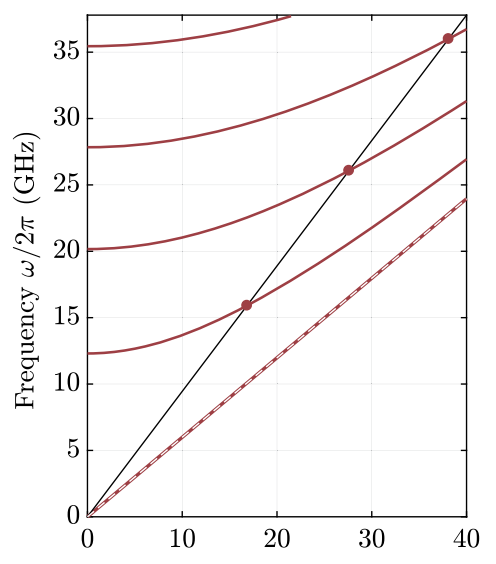

(a) $\mathrm{T}_{0 n}$ bands at $j=0$

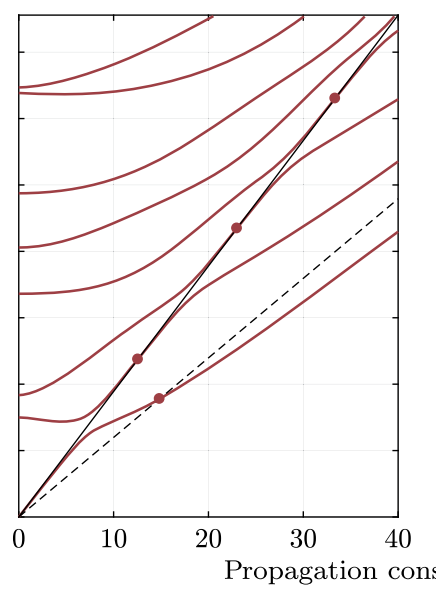

(b) $\mathrm{L}_{0 n}$ bands at $j=0$

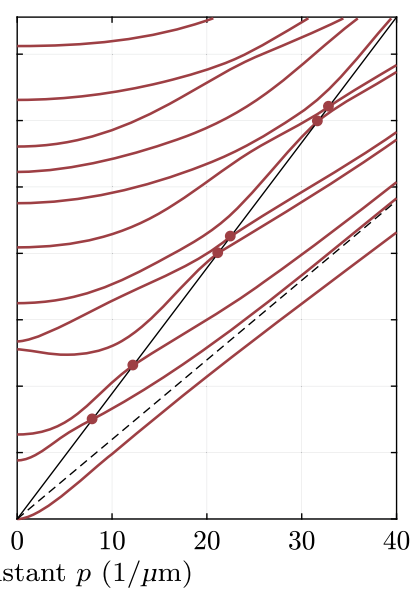

(c) $\mathrm{F}_{1 n}$ bands at $j= \pm 1$

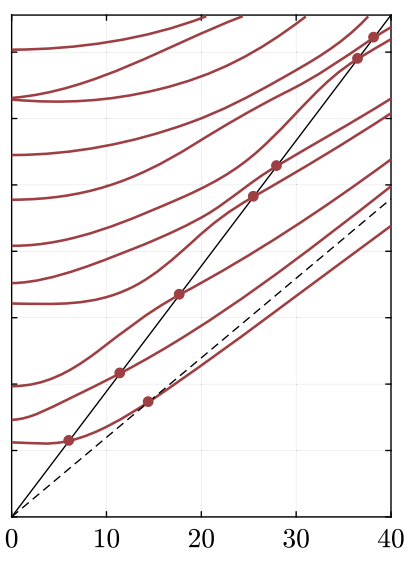

(d) $\mathrm{F}_{2 n}$ bands at $j= \pm 2$

FIG. 5. Band structures of nanofiber phonon modes with different azimuthal order $j$, for radius $R$ and mechanical properties specified in Appendix E. Panel (a) shows torsional bands, panel (b) longitudinal bands, and panel (c) flexural bands of azimuthal order $m= \pm 1$ and $m= \pm 2$, respectively. The solid black line delineates the longitudinal sound line, the dashed line the transverse sound line [which coincides with the lowest band in Fig. 5(a)]. Solid dots mark intersections of phonon bands with either sound line.

confined by the finite fiber radius, there is only a discrete set of frequencies $\omega_{\gamma}$ admissible for each $(j, p)$, analogous to the case of guided photonic fiber modes. The eigenfrequencies $\omega_{\gamma}(p)$ form discrete bands in the $(p, \omega)$ plane; see Fig. 5. We can therefore count radial excitations using a discrete band index $n \in \mathbb{N}$ starting from $n=1$ for the band of lowest frequency and increasing in frequency with $n$. Since the propagation constant is the only continuous mode index, the orthonormality relation Eq. (C7) reduces to a normalization condition for the radial partial waves:

$$
\int_{0}^{R} r\left|\mathcal{W}_{\gamma}(r)\right|^{2} d r=1
$$

Elastodynamics, unlike electrodynamics, allows for longitudinal in addition to transverse polarizations even in the absence of surfaces. In the nanofiber, the presence of a surface forces these excitations to hybridize, forming eigenmodes that can have both transverse and longitudinal contributions. Transverse waves propagate with the transverse sound velocity $c_{t}$, while longitudinal waves propagate with the longitudinal sound velocity $c_{l}$, which is typically larger than the transverse sound velocity:

$$
\begin{aligned}
c_{t} & \equiv \sqrt{\frac{E}{2 \rho(1+\nu)},} \\
c_{l} & \equiv \sqrt{\frac{E(\nu-1)}{\rho\left(\nu+2 \nu^{2}-1\right)}} .
\end{aligned}
$$

The radial partial waves $\mathcal{W}_{\gamma}$ have three contributions,

$$
\mathcal{W}_{\gamma}(r)=A \mathcal{W}^{a}(r)+B \mathcal{W}^{b}(r)+C \mathcal{W}^{c}(r)
$$

where $A, B, C \in \mathbb{C}$ are amplitudes. The components of the three vectorial terms $\mathcal{W}^{a}, \mathcal{W}^{b}$, and $\mathcal{W}^{c}$ can be expressed in terms of quantities defined in Table IX and are listed in Table X. The form of the eigenmodes depends on the magnitude of the eigenfrequency $\omega_{\gamma}$ compared to the longitudinal sound line $\omega_{l} \equiv c_{l}|p|$ and the transverse sound line $\omega_{t} \equiv c_{t}|p|$. We assume that $c_{l}>c_{t}$ in the following discussion, as is the case for most materials. If this is not the case, the radial partial waves of eigenmodes are different from the ones given in this Appendix.

The eigenmodes have to meet the boundary conditions discussed above to ensure a stress-free surface. In terms of the stress modal field

TABLE IX. Definitions of the longitudinal and the transverse sound velocity $c_{l}$ and $c_{t}$, as well as the radial constants $a$ and $b$, and the dimensionless quantities appearing in the phonon modal

\begin{tabular}{|c|c|}
\hline$c_{t}=\sqrt{E / 2 \rho(1+\nu)}$ & $c_{l}=\sqrt{E(\nu-1) / \rho\left(\nu+2 \nu^{2}-1\right)}$ \\
\hline$a=\sqrt{\omega^{2} / c_{t}^{2}-p^{2}}$ & $\tilde{a}=-i a$ \\
\hline$b=\sqrt{\omega^{2} / c_{l}^{2}-p^{2}}$ & $\tilde{b}=-i b$ \\
\hline$\omega_{t}=c_{t}|p|$ & $\omega_{l}=c_{l}|p|$ \\
\hline$\alpha=a R$ & $\tilde{\alpha}=\tilde{a} R$ \\
\hline$\beta=b R$ & $\tilde{\beta}=\tilde{b} R$ \\
\hline$\varpi=p R$ & $x=r / R$ \\
\hline $\begin{array}{l}\eta=J_{1}(\beta) / J_{1}(\alpha) \\
\breve{\eta}=I_{1}(\tilde{\beta}) / I_{1}(\tilde{\alpha})\end{array}$ & $\tilde{\eta}=I_{1}(\tilde{\beta}) / J_{1}(\alpha)$ \\
\hline$\sigma_{p}=p /|p|$ & $\sigma_{j}=j /|j|$ \\
\hline
\end{tabular}
fields. The definitions are given in terms of the density $\rho$, Young's modulus $E$, Poisson ration $\nu$, fiber radius $R$, azimuthal order $j$, propagation constant $p$, and radial position $r$. 
TABLE X. Fiber eigenmodes: Terms in the radial partial wave $\mathcal{W}_{\gamma}$ of the displacement eigenmode in Eq. (C16). The radial dependence is given by Bessel functions $J_{j}$ and modified Bessel functions $I_{j}$ of the first kind. The prime indicates the first derivative $J_{j}^{\prime}(x)=\partial_{x} J_{j}(x)$. All remaining quantities are defined in Table IX.

\begin{tabular}{ccccc}
\hline \hline \multirow{2}{*}{ Term } & Case & $k=r$ & $k=\varphi$ & $k=z$ \\
\cline { 3 - 5 } $\mathcal{W}^{k a}$ & $\omega_{\gamma}>\omega_{l}$ & $\beta J_{j}^{\prime}(\beta x)$ & $i j J_{j}(\beta x) / x$ & $i \varpi J_{j}(\beta x)$ \\
& $\omega_{\gamma}=\omega_{l}$ & $|j| x^{|j|-1}$ & $i j x^{|j|-1}$ & $i \varpi x^{|j|}$ \\
& $\omega_{\gamma}<\omega_{l}$ & $\tilde{\beta} I_{j}^{\prime}(\tilde{\beta} x)$ & $i j I_{j}(\tilde{\beta} x) / x$ & $i \varpi I_{j}(\tilde{\beta} x)$ \\
$\mathcal{W}^{k b}$ & $\omega_{\gamma}>\omega_{t}$ & $\varpi J_{j+1}(\alpha x)$ & $-i \varpi J_{j+1}(\alpha x)$ & $i \alpha J_{j}(\alpha x)$ \\
& $\omega_{\gamma}=\omega_{t}$ & $\varpi x^{|j|+1}$ & $-\sigma_{j} i \varpi x^{|j|+1}$ & $2 i(|j|+1) x^{|j|}$ \\
& $\omega_{\gamma}<\omega_{t}$ & $\varpi I_{j+1}(\tilde{\alpha} x)$ & $-i \varpi I_{j+1}(\tilde{\alpha} x)$ & $i \tilde{\alpha} I_{j}(\tilde{\alpha} x)$ \\
$\mathcal{W}^{k c}$ & $\omega_{\gamma}>\omega_{t}$ & $j J_{j}(\alpha x) / x$ & $i \alpha J_{j}{ }^{\prime}(\alpha x)$ & 0 \\
& $\omega_{\gamma}=\omega_{t}$ & $\varpi x^{|j|-1}$ & $\sigma_{j} i \varpi x^{|j|-1}$ & 0 \\
& $\omega_{\gamma}<\omega_{t}$ & $j I_{j}(\tilde{\alpha} x) / x$ & $i \tilde{\alpha} I_{j}{ }^{\prime}(\tilde{\alpha} x)$ & 0 \\
\hline \hline
\end{tabular}

$$
t_{\gamma}^{k l}(\boldsymbol{r}) \equiv \sum_{m n} C^{k l m n} s_{\gamma}^{m n}(\boldsymbol{r})
$$

defined here using the strain modal field Eq. (C11), the boundary conditions are $\boldsymbol{t}_{\gamma} \boldsymbol{n}=\mathbf{0}$ at $r=R$. For the nanofiber, the exterior surface normal vector is $\boldsymbol{n}=\boldsymbol{e}_{r}$. The stress modal field can be decomposed into partial waves

$$
\boldsymbol{t}_{\gamma}(\boldsymbol{r})=\frac{\mathcal{T}_{\gamma}(r)}{2 \pi} e^{i(j \varphi+i p z)},
$$

and the radial partial waves of the relevant components brought into the form

$$
\begin{aligned}
& \mathcal{T}_{\gamma}^{r r}(r)=-\frac{2 \mu R}{r^{2}}\left[A M^{r a}(r)+B M^{r b}(r)+C M^{r c}(r)\right], \\
& \mathcal{T}_{\gamma}^{\varphi r}(r)=i \frac{\mu R}{r^{2}}\left[A M^{\varphi a}(r)+B M^{\varphi b}(r)+C M^{\varphi c}(r)\right], \\
& \mathcal{T}_{\gamma}^{z r}(r)=i \frac{\mu R}{r^{2}}\left[A M^{z a}(r)+B M^{z b}(r)+C M^{z c}(r)\right] .
\end{aligned}
$$

The nine components $M^{k l}, k=r, \varphi, z, l=a, b, c$ evaluated on the fiber surface $r=R$ are listed in Table XI. Let $\boldsymbol{M}$ be the matrix with coefficients $M^{k l}(r=R)$. The boundary conditions can then be written as

$$
\boldsymbol{M}\left(\begin{array}{l}
A \\
B \\
C
\end{array}\right)=\mathbf{0}
$$

and yield relations between the three amplitudes $A, B, C$. For each mode family, one independent amplitude remains, which can subsequently be determined from the normalization condition Eq. (C14). The trivial solution $A=B=$ $C=0$ corresponds to zero displacement and is of no interest to us. The boundary conditions Eq. (C20) can be met with nontrivial amplitudes if and only if

$$
\operatorname{det} \boldsymbol{M}=0 .
$$

This relation is the frequency equation for the eigenmodes, as it constrains the admissible eigenfrequencies $\omega_{\gamma}$ for a given propagation constant $p$ and azimuthal order $j$. It is therefore an implicit equation for the dispersion relation $\omega_{\gamma}(p)$.

We distinguish the cases $j=0$ and $|j| \geq 1$. For azimuthal order $j=0, M^{r c}=M^{\varphi a}=M^{z c}=0$, and the fre-

\begin{tabular}{|c|c|c|c|c|}
\hline \multirow[b]{2}{*}{ Term } & \multicolumn{4}{|c|}{ Component } \\
\hline & Case & $k=r$ & $k=\varphi$ & $k=z$ \\
\hline$M^{k a}$ & $\begin{array}{l}\omega_{\gamma}>\omega_{l}>\omega_{t} \\
\omega_{\gamma}=\omega_{l}>\omega_{t} \\
\omega_{l}>\omega_{\gamma}>\omega_{t} \\
\omega_{l}>\omega_{\gamma}=\omega_{t} \\
\omega_{l}>\omega_{t}>\omega_{\gamma}\end{array}$ & $\begin{array}{c}{\left[\left(\alpha^{2}-\varpi^{2}\right) / 2-\left(j^{2}-j\right)\right] J_{j}(\beta)-\beta J_{j+1}(\beta)} \\
{\left[\left(\alpha^{2}-\varpi^{2}\right) / 2-\left(j^{2}-|j|\right)\right]} \\
{\left[\left(\alpha^{2}-\varpi^{2}\right) / 2-\left(j^{2}-j\right)\right] I_{j}(\tilde{\beta})+\tilde{\beta} I_{j+1}(\tilde{\beta})} \\
{\left[-\varpi^{2} / 2-\left(j^{2}-j\right)\right] I_{j}(\tilde{\beta})+\tilde{\beta} I_{j+1}(\tilde{\beta})} \\
{\left[-\left(\tilde{\alpha}^{2}+\varpi^{2}\right) / 2-\left(j^{2}-j\right)\right] I_{j}(\tilde{\beta})+\tilde{\beta} I_{j+1}(\tilde{\beta})}\end{array}$ & $\begin{array}{l}2\left(j^{2}-j\right) J_{j}(\beta)-2 j \beta J_{j+1}(\beta) \\
2 j(|j|-1) \\
2\left(j^{2}-j\right) I_{j}(\tilde{\beta})+2 j \tilde{\beta} I_{j+1}(\tilde{\beta}) \\
2\left(j^{2}-j\right) I_{j}(\tilde{\beta})+2 j \tilde{\beta} I_{j+1}(\tilde{\beta}) \\
2\left(j^{2}-j\right) I_{j}(\tilde{\beta})+2 j \tilde{\beta} I_{j+1}(\tilde{\beta})\end{array}$ & $\begin{array}{l}2 j \varpi I_{j}(\tilde{\beta})+2 \varpi \tilde{\beta} I_{j+1}(\tilde{\beta}) \\
2|j| \varpi \\
2 j \varpi I_{j}(\tilde{\beta})+2 \varpi \tilde{\beta} I_{j+1}(\tilde{\beta}) \\
2 j \varpi I_{j}(\tilde{\beta})+2 \varpi \tilde{\beta} I_{j+1}(\tilde{\beta}) \\
2 j \varpi I_{j}(\tilde{\beta})+2 \varpi \tilde{\beta} I_{j+1}(\tilde{\beta})\end{array}$ \\
\hline$M^{k b}$ & $\begin{array}{l}\omega_{\gamma}>\omega_{t} \\
\omega_{\gamma}=\omega_{t} \\
\omega_{\gamma}<\omega_{t}\end{array}$ & $\begin{array}{c}-\varpi \alpha J_{j}(\alpha)+(j+1) \varpi J_{j+1}(\alpha) \\
-(|j|+1) \varpi \\
-\varpi \tilde{\alpha} I_{j}(\tilde{\alpha})+(j+1) \varpi I_{j+1}(\tilde{\alpha})\end{array}$ & $\begin{array}{c}-\varpi \alpha J_{j}(\alpha)+2(j+1) \varpi J_{j+1}(\alpha) \\
0 \\
-\varpi \tilde{\alpha} I_{j}(\tilde{\alpha})+2(j+1) \varpi I_{j+1}(\tilde{\alpha})\end{array}$ & $\begin{array}{c}j \alpha J_{j}(\alpha)+\left(\varpi^{2}-\alpha^{2}\right) J_{j+1}(\alpha) \\
2\left(j^{2}+|j|\right)+\varpi^{2} \\
j \tilde{\alpha} I_{j}(\tilde{\alpha})+\left(\varpi^{2}+\tilde{\alpha}^{2}\right) I_{j+1}(\tilde{\alpha})\end{array}$ \\
\hline$M^{k c}$ & $\begin{array}{l}\omega_{\gamma}>\omega_{t} \\
\omega_{\gamma}=\omega_{t} \\
\omega_{\gamma}<\omega_{t}\end{array}$ & $\begin{array}{c}\left(j-j^{2}\right) J_{j}(\alpha)+j \alpha J_{j+1}(\alpha) \\
-\left(1-\delta_{j 0}\right)(|j|-1) \varpi \\
\left(j-j^{2}\right) I_{j}(\tilde{\alpha})-j \tilde{\alpha} I_{j+1}(\tilde{\alpha}) \\
\end{array}$ & $\begin{array}{c}{\left[2\left(j^{2}-j\right)-\alpha^{2}\right] J_{j}(\alpha)+2 \alpha J_{j+1}(\alpha)} \\
2\left(1-\delta_{j 0}\right)\left(j-\sigma_{j}\right) \varpi \\
{\left[2\left(j^{2}-j\right)+\tilde{\alpha}^{2}\right] I_{j}(\tilde{\alpha})-2 \tilde{\alpha} I_{j+1}(\tilde{\alpha})}\end{array}$ & $\begin{array}{c}j \varpi J_{j}(\alpha) \\
\left(1-\delta_{j 0}\right) \varpi^{2} \\
j \varpi I_{j}(\tilde{\alpha})\end{array}$ \\
\hline
\end{tabular}
quency equation (C21) factorizes,

$$
M^{\varphi c} \operatorname{det}\left(\begin{array}{ll}
M^{r a} & M^{r b} \\
M^{z a} & M^{z b}
\end{array}\right)=0
$$

TABLE XI. Fiber eigenmodes: Terms in the radial partial wave $\mathcal{T}_{\gamma}$ of the stress modal field in Eq. (C19). The terms are evaluated on the fiber surface $(r=R)$ and correspond to the matrix elements of $\boldsymbol{M}$ in the boundary condition Eq. (C20) and frequency equation (C21). All remaining quantities are defined in Table IX. 


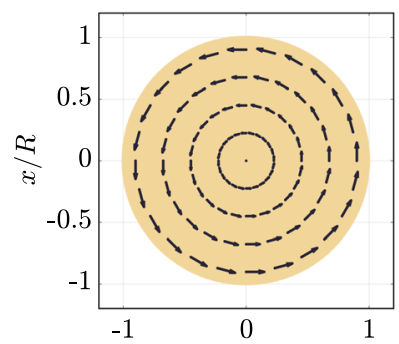

(a) $\mathrm{T}_{01}$ at $z=\lambda / 4$

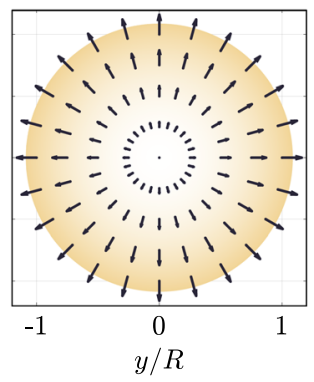

(b) $\mathrm{L}_{01}$ at $z=0$

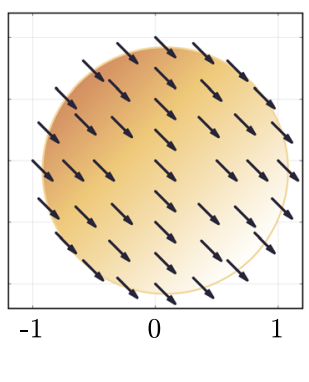

(c) $\mathrm{F}_{11}$ at $z=\lambda / 8$

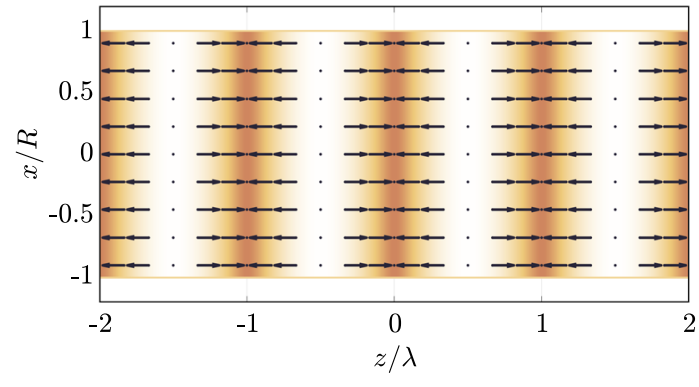

(d) $\mathrm{L}_{01}$ at $y=0$

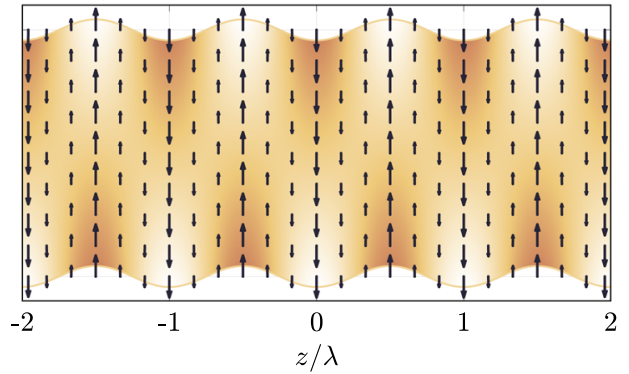

(e) $\mathrm{F}_{01}$ at $y=0$

FIG. 6. Mechanical nanofiber eigenmodes on the three fundamental bands. Panels (a)-(c) show a cross section of the nanofiber, panels (d) and (e) a longitudinal section. The arrows correspond to the displacement field of a single mode with arbitrary amplitude $\beta_{\gamma}$; see Eq. (C8). The color gradients show density variations $\nabla \cdot \boldsymbol{u}$ displayed at $\boldsymbol{r}+\boldsymbol{u}(\boldsymbol{r})$ to simultaneously indicate the displacement of the fiber section. Darker areas are of higher density than lighter areas. Displacements in the vector plot are exaggerated by a factor of 2 compared to the density plot. The mechanical properties of the fiber are given in Appendix E. All three phonon modes have frequency $\omega=2 \pi \times 123 \mathrm{kHz}$ resonant with the radial atom trap; see Sec. III. The wavelengths $\lambda=2 \pi / p$ are much larger than the nanofiber radius and vary between modes. The longitudinal sections therefore do not preserve the aspect ratio of the fiber: The $z$ axis is compressed by a factor of $\lambda / R \simeq 4 \times 10^{5}$ relative to the $x$ axis for the $\mathrm{L}$ mode in (d) and by $\lambda / R \simeq 2 \times 10^{3}$ for the $\mathrm{F}$ mode in (e), while both components of the displacement field are drawn to the same scale. The $\mathrm{T}_{01}$ mode in (a) leads only to azimuthal displacement and does not induce density variations. The $\mathrm{L}_{01}$ has a dominant longitudinal component resulting in density waves along the fiber axis; see (d). Its radial component causes breathing of the fiber radius and is smaller by a factor $c_{h} /(\omega R \nu) \simeq 1.8 \times 10^{5}$. The radial component in (b) is exaggerated by a corresponding factor compared to the axial component in (d) in order to be visible. The $\mathrm{F}_{11}$ mode displaces the entire fiber cross section orthogonal to the fiber axis in a circular motion; see (c). The density variations in (c) and (e) result from longitudinal displacement that is smaller than the transverse displacement visible in the vector plot by a factor of $\sqrt{c_{h} /(2 \omega R)} \simeq 120$.

giving rise to two independent mode families: torsional $(\mathrm{T})$ modes with $M^{\varphi c}=0$ and longitudinal (L) modes for which the determinant in Eq. (C22) vanishes. For higher azimuthal excitations $|j| \geq 1$, this is not the case, and there is only one family of flexural $(\mathrm{F})$ modes.

Torsional modes can exist only above and on the transverse sound line $\omega_{t}$; see Fig. 5(a). Longitudinal and flexural modes, on the other hand, are hybrids of longitudinal and transverse waves, and exhibit different behavior depending on the magnitude of their frequency $\omega_{\gamma}$ compared to the transverse sound line $\omega_{t}$ and the longitudinal sound line $\omega_{l}$. In case the frequency lies above the longitudinal sound line $\omega_{\gamma}>\omega_{l}>\omega_{t}$, both transverse and longitudinal excitations can propagate in the bulk of the cylinder, and the eigenmode shows oscillatory behavior in $r$. We refer to these modes as bulk modes. In case the frequency lies between the two sound lines $\omega_{l}>\omega_{\gamma}>\omega_{t}$, we call the modes mixed modes: Only the transverse contributions to the eigenmodes oscillate in $r$, while the longitudinal contributions decay as modified Bessel functions of first kind $I_{n}$ away from the surface towards the center of the cylinder. Surface modes are characterized by $\omega_{l}>\omega_{t}>\omega_{\gamma}$. Neither transverse nor longitudinal excitations can propagate in the bulk of the fiber, and eigenmodes are confined to the fiber surface. Such modes are often called surface acoustic waves $[58,110]$. On each sound line, the respective contributions to the eigenmodes are polynomial in $r$.

Torsional modes are characterized by $j=0$ and the frequency equation $M^{\varphi c}=0$. They have zero longitudinal and radial displacement $w_{\gamma}^{r}=w_{\gamma}^{z}=0$, and are therefore purely transverse excitations; see Fig. 6(a). The 
TABLE XII. Torsional (T) fiber eigenmodes: Nonzero component of the displacement eigenmode and frequency equations. The amplitude $C \in \mathbb{C}$ is fixed by the normalization condition Eq. (C14). All quantities are defined in Table IX.

\begin{tabular}{lcc}
\hline \hline Case & Eigenmode component & Frequency equation \\
\hline$\omega_{\gamma}>\omega_{t}$ & $\mathcal{W}_{\gamma}^{\varphi}(r)=C J_{1}(\alpha x)$ & $J_{2}(\alpha)=0$ \\
$\omega_{\gamma}=\omega_{t}$ & $\mathcal{W}_{\gamma}^{\varphi}(r)=C x$ & $\omega_{\gamma}(p)=c_{t}|p|$ \\
\hline \hline
\end{tabular}

radial partial waves of the eigenmode and the frequency equations are listed in Table XII. The roots of the frequency equation form bands $\mathrm{T}_{0 n}$ in the $(p, \omega)$ plane plotted in Fig. 5(a).

On the transverse sound line $\left(\omega_{\gamma}=\omega_{t}\right)$, the frequency equation is always satisfied, while it has no solution below the transverse sound line $\left(\omega_{\gamma}<\omega_{t}\right)$. The fundamental (i.e., lowest frequency) torsional band $\mathrm{T}_{01}$ thus coincides with the transverse sound line. The radial partial wave of the strain modal field on the $\mathrm{T}_{01}$ band has two nonzero components:

$$
\mathcal{S}_{\gamma}^{\varphi z}(r)=\mathcal{S}_{\gamma}^{z \varphi}(r)=\frac{i}{2} \frac{C}{R} \varpi x
$$

see Table IX for definitions of the symbols.

Longitudinal modes are characterized by $j=0$ and the frequency equation

$$
\operatorname{det}\left(\begin{array}{ll}
M^{r a} & M^{r b} \\
M^{z a} & M^{z b}
\end{array}\right)=0 .
$$

They have zero azimuthal displacement $w_{\gamma}^{\varphi}=0$ and are indeed largely longitudinal excitations similar to sound waves, but they do have a small nonzero radial component; see Figs. 6(b) and 6(d). Longitudinal modes exist in the bulk, mixed, and surface mode sector, and cross both sound lines. The radial partial wave of the displacement field is given explicitly in Table XIII for all five cases. These expressions hold as long as $M^{\varphi c} \neq 0$ (i.e., provided the mode is not located at a crossing with a torsional band). Otherwise, the eigenmode can be obtained from the general expressions in Table $\mathrm{X}$ by solving the boundary conditions Eq. (C20). The frequency equations obtained from Eq. (C24) are listed in Table XIV and are known as Pochhammer equations. The roots of the Pochhammer equations form bands $\mathrm{L}_{0 n}$ in the $(p, \omega)$ plane, as shown in Fig. 5(b).

The fundamental longitudinal band $\mathrm{L}_{01}$ lies in the mixedmode sector in the low-frequency limit. In this case, the radial partial wave of the strain modal field has components

$$
\begin{aligned}
& \mathcal{S}_{\gamma}^{r r}(r)=-\tilde{\beta}^{2} \frac{A}{R}\left[\frac{2 \varpi^{2} \tilde{\eta}}{\varpi^{2}-\alpha^{2}} \frac{\alpha}{\tilde{\beta}} J_{1}^{\prime}(\alpha x)-I_{1}^{\prime}(\tilde{\beta} x)\right], \\
& \mathcal{S}_{\gamma}^{\varphi \varphi}(r)=-\tilde{\beta} \frac{A}{R}\left[\frac{2 \varpi^{2} \tilde{\eta}}{\varpi^{2}-\alpha^{2}} \frac{J_{1}(\alpha x)}{x}-\frac{I_{1}(\tilde{\beta} x)}{x}\right], \\
& \mathcal{S}_{\gamma}^{z z}(r)=\varpi^{2} \frac{A}{R}\left[\frac{2 \alpha \tilde{\beta} \tilde{\eta}}{\varpi^{2}-\alpha^{2}} J_{0}(\alpha x)-I_{0}(\tilde{\beta} x)\right], \\
& \mathcal{S}_{\gamma}^{r z}(r)=-i \tilde{\beta} \varpi \frac{A}{R}\left[\tilde{\eta} J_{1}(\alpha x)-I_{1}(\tilde{\beta} x)\right],
\end{aligned}
$$

TABLE XIII. Longitudinal (L) fiber eigenmodes: Nonzero components of the radial partial wave of the displacement eigenmode. The amplitude $A \in \mathbb{C}$ can be obtained from the normalization condition (C14), all other quantities are defined in Table IX. These expressions are valid as long as $M^{\varphi c} \neq 0$, that is, away from intersections with torsional bands. Otherwise, the displacement eigenmode can be obtained from the general expressions in Table $\mathrm{X}$ in conjunction with Eq. (C20).

\begin{tabular}{lll}
\hline \hline & \multicolumn{1}{c}{ Eigenmode component } \\
\cline { 2 - 3 } Case & \multicolumn{1}{c}{$k=r$} & \multicolumn{1}{c}{$k=z$} \\
\hline$\omega_{\gamma}>\omega_{l}>\omega_{t}$ & $\beta A\left[2 \varpi^{2} \eta J_{1}(\alpha x) /\left(\varpi^{2}-\alpha^{2}\right)-J_{1}(\beta x)\right]$ & $i \varpi A\left[2 \alpha \beta \eta J_{0}(\alpha x) /\left(\varpi^{2}-\alpha^{2}\right)+J_{0}(\beta x)\right]$ \\
$\omega_{\gamma}=\omega_{l}>\omega_{t}$ & $A\left(\alpha^{2}-\varpi^{2}\right) J_{1}(\alpha x) /\left[2 \alpha J_{0}(\alpha)\right]$ & $i \varpi A\left\{1+\left(\alpha^{2}-\varpi^{2}\right) J_{0}(\alpha x) /\left[2 \varpi^{2} J_{0}(\alpha)\right]\right\}$ \\
$\omega_{l}>\omega_{\gamma}>\omega_{t}$ & $\tilde{\beta} A\left[-2 \varpi^{2} \tilde{\eta} /\left(\varpi^{2}-\alpha^{2}\right) J_{1}(\alpha x)+I_{1}(\tilde{\beta} x)\right]$ & $i \varpi A\left[-2 \alpha \tilde{\beta} \tilde{\eta} /\left(\varpi^{2}-\alpha^{2}\right) J_{0}(\alpha x)+I_{0}(\tilde{\beta} x)\right]$ \\
$\omega_{l}>\omega_{\gamma}=\omega_{t}$ & $\tilde{\beta} A\left[I_{1}(\tilde{\beta} x)-2 I_{1}(\tilde{\beta}) x\right]$ & $i A\left[\varpi I_{0}(\tilde{\beta} x)-4 \tilde{\beta} I_{1}(\tilde{\beta}) / \varpi\right]$ \\
$\omega_{l}>\omega_{t}>\omega_{\gamma}$ & $\tilde{\beta} A\left[-2 \varpi^{2} \breve{\eta} I_{1}(\tilde{\alpha} x) /\left(\varpi^{2}+\tilde{\alpha}^{2}\right)+I_{1}(\tilde{\beta} x)\right]$ & $i \varpi A-2 \tilde{\alpha} \tilde{\beta} \breve{\eta} I_{0}(\tilde{\alpha} x) /\left(\varpi^{2}+\tilde{\alpha}^{2}\right)+I_{0}(\tilde{\beta} x)$ \\
\hline \hline
\end{tabular}

TABLE XIV. Longitudinal (L) fiber eigenmodes: Frequency equations. All quantities are defined in Table IX.

\begin{tabular}{lc}
\hline \hline Case & Frequency equation \\
\hline$\omega_{\gamma}>\omega_{l}>\omega_{t}$ & $0=2 \beta\left(\alpha^{2}+\varpi^{2}\right) J_{1}(\alpha) J_{1}(\beta)-\left(\alpha^{2}-\varpi^{2}\right)^{2} J_{1}(\alpha) J_{0}(\beta)-4 \alpha \beta \varpi^{2} J_{0}(\alpha) J_{1}(\beta)$ \\
$\omega_{\gamma}=\omega_{l}>\omega_{t}$ & $0=J_{1}(\alpha)$ \\
$\omega_{l}>\omega_{\gamma}>\omega_{t}$ & $0=-2 \tilde{\beta}\left(\alpha^{2}+\varpi^{2}\right) J_{1}(\alpha) I_{1}(\tilde{\beta})-\left(\alpha^{2}-\varpi^{2}\right)^{2} J_{1}(\alpha) I_{0}(\tilde{\beta})+4 \alpha \tilde{\beta} \varpi^{2} J_{0}(\alpha) I_{1}(\tilde{\beta})$ \\
$\omega_{l}>\omega_{\gamma}=\omega_{t}$ & $0=\varpi^{2} I_{0}(\tilde{\beta})-6 \tilde{\beta} I_{1}(\tilde{\beta})$ \\
$\omega_{l}>\omega_{t}>\omega_{\gamma}$ & $0=2 \tilde{\beta}\left(\tilde{\alpha}^{2}-\varpi^{2}\right) I_{1}(\tilde{\alpha}) I_{1}(\tilde{\beta})-\left(\tilde{\alpha}^{2}+\varpi^{2}\right)^{2} I_{1}(\tilde{\alpha}) I_{0}(\tilde{\beta})+4 \tilde{\alpha} \tilde{\beta} \varpi^{2} I_{0}(\tilde{\alpha}) I_{1}(\tilde{\beta})$ \\
\hline \hline
\end{tabular}


TABLE XV. Fiber eigenmodes: Components of the radial partial wave $\mathcal{S}_{\gamma}$ of the strain modal field for the surface mode sector $\omega<\omega_{t}$. The amplitudes $A, B, C$ are determined by the boundary conditions Eq. (C20) and the normalization condition Eq. (C14). All remaining quantities are defined in Table IX.

\begin{aligned} & \hline \hline $\mathcal{S}_{\gamma}^{r r}=\left\{B \varpi \tilde{\alpha} I_{j}(\tilde{\alpha} x)+C j(j-1) I_{j}(\tilde{\alpha} x) / x^{2}-[B(j+1) \varpi-C j \tilde{\alpha}] I_{j+1}(\tilde{\alpha} x) / x+A \tilde{\beta}^{2} I_{j}(\tilde{\beta} x)+A j(j-1) I_{j}(\tilde{\beta} x) / x^{2}-A \tilde{\beta} I_{j+1}(\tilde{\beta} x) / x\right\} / R \\ & \mathcal{S}_{\gamma}^{\varphi \varphi}=\left\{-C j(j-1) I_{j}(\tilde{\alpha} x) / x^{2}+[B(j+1) \varpi-C j \tilde{\alpha}] I_{j+1}(\tilde{\alpha} x) / x-A j(j-1) I_{j}(\tilde{\beta} x) / x^{2}+A \tilde{\beta} I_{j+1}(\tilde{\beta} x) / x\right\} / R \\ & \mathcal{S}_{\gamma}^{z z}=\left[-B \varpi \tilde{\alpha} I_{j}(\tilde{\alpha} x)-A \varpi^{2} I_{j}(\tilde{\beta} x)\right] / R \\ & \mathcal{S}_{\gamma}^{r \varphi}=\left\{-i\left(B \varpi \tilde{\alpha}-C \tilde{\alpha}^{2}\right) I_{j}(\tilde{\alpha} x) / 2+i C j(j-1) I_{j}(\tilde{\alpha} x) / x^{2}+i[B(j+1) \varpi-C \tilde{\alpha}] I_{j+1}(\tilde{\alpha} x) / x+i A j(j-1) I_{j}(\tilde{\beta} x) / x^{2}+i A j \tilde{\beta} I_{j+1}(\tilde{\beta} x) / x\right\} / R \\ & \mathcal{S}_{\gamma}^{r z}=\left[i j(B \tilde{\alpha}+C \varpi) I_{j}(\tilde{\alpha} x) / 2 x+i B\left(\tilde{\alpha}^{2}+\varpi^{2}\right) I_{j+1}(\tilde{\alpha} x) / 2+i j A \varpi I_{j}(\tilde{\beta} x) / x+i A \varpi \tilde{\beta} I_{j+1}(\tilde{\beta} x)\right] / R \\ & \mathcal{S}_{\gamma}^{\varphi z}=\left[-j(B \tilde{\alpha}+C \varpi) I_{j}(\tilde{\alpha} x) / 2 x+\left(B \varpi^{2}-C \varpi \tilde{\alpha}\right) I_{j+1}(\tilde{\alpha} x) / 2-A j \varpi I_{j}(\tilde{\beta} x) / x\right] / R \\ &$\hline\end{aligned}

while the remaining independent components vanish. Refer to Table IX for definitions of the symbols.

Flexural modes appear for azimuthal orders $|j| \geq 0$. In this case, the frequency equation (C21) does not in general factorize. There is then only one family of flexural modes, with a displacement field specified in Eqs. (C13) and (C16), as well as Table X. A flexural mode with azimuthal order $|j|=1$ is shown in Figs. 6(c) and 6(e). The boundary conditions Eq. (C20) enable us to relate two of the three amplitudes $A, B, C$ to the third one. For instance,

$$
\begin{aligned}
& B=\frac{M^{r c} M^{\varphi a}-M^{r a} M^{\varphi c}}{M^{r b} M^{\varphi c}-M^{r c} M^{\varphi b}} A, \\
& C=\frac{M^{r a} M^{\varphi b}-M^{r b} M^{\varphi a}}{M^{r b} M^{\varphi c}-M^{r c} M^{\varphi b}} A,
\end{aligned}
$$

and the remaining amplitude $A$ is fixed by the normalization condition Eq. (C14) [111].

The roots of the frequency equation $\operatorname{det} \boldsymbol{M}=0$ with matrix components listed in Table XI form bands $\mathrm{F}_{|j| n}$ in the $(p, \omega)$ plane. In Figs. 5(c) and 5(d), the $\mathrm{F}_{1 n}$ and $\mathrm{F}_{2 n}$ bands are shown. The fundamental flexural band $\mathrm{F}_{01}$ lies in the surface mode sector. The radial partial waves of the strain modal field in this case are listed in Table XV.

In summary, we can label the phononic eigenmodes of a fiber with indices

$$
\begin{array}{ll}
j \in \mathbb{Z}, & f \in\{\mathrm{L}, \mathrm{T}\}_{j=0} \quad \text { or } \quad\{\mathrm{F}\}_{j \neq 0}, \\
p \in \mathbb{R}, & n \in \mathbb{N} .
\end{array}
$$

Following the conventions used for photonic fiber eigenmodes, we label the different phonon bands by their mode indices as $f_{|j| n}$. At azimuthal order $j=0$, there are then $\mathrm{T}_{0 n}$ and $\mathrm{L}_{0 n}$ bands shown in Figs. 5(a) and 5(b). At azimuthal order $|j| \geq 1$, there are $\mathrm{F}_{|j| n}$ bands plotted in Figs. 5(c) and 5(d) for $|j|=1,2$.

Figure 5 shows that there are three fundamental bands without a finite minimum frequency: $\mathrm{L}_{01}, \mathrm{~T}_{01}$, and $\mathrm{F}_{11}$. Nanofiber-based cold-atom traps have trap frequencies on the order of $100 \mathrm{kHz}[5,9,24,76]$ as we discuss in Sec. III, so only modes on the fundamental bands can resonantly couple to the atoms for typical parameters of the nanofiber.
The fundamental bands are therefore of special importance in this article, and we provide approximate expressions for the dispersion relations and displacement fields in the lowfrequency limit. The band $\mathrm{T}_{01}$ [see Fig. 5(a)] lies on the transverse sound line and is thus given by the dispersion relation

$$
\omega_{T}(p)=c_{t}|p| .
$$

The only nonzero component of the displacement eigenmode normalized according to Eq. (C14) is

$$
\mathcal{W}_{\gamma}^{\varphi}(r)=\frac{2}{R^{2}} r .
$$

The displacement induced by a $\mathrm{T}_{01}$ mode is shown in Fig. 6(a). The band $\mathrm{L}_{01}$ [see Fig. 5(b)] lies in the mixedmode sector for low frequencies. The exact dispersion relation is the solution to the transcendental Pochhammer equation given in Table XIV. It has the linear asymptote

$$
\omega_{L}(p) \simeq c_{h}|p|,
$$

with an effective hybrid speed of sound

$$
c_{h} \equiv \sqrt{\frac{E}{\rho}} .
$$

The components of the normalized radial partial waves approximated to linear order in $p r \ll 1$ for wavelengths much larger than the fiber radius are

$$
\begin{aligned}
& \mathcal{W}_{\gamma}^{r}(r) \simeq \frac{\sqrt{2} \nu p}{R} r, \\
& \mathcal{W}_{\gamma}^{z}(r) \simeq i \frac{\sqrt{2}}{R},
\end{aligned}
$$

while the azimuthal component vanishes. An $\mathrm{L}_{01}$ mode is plotted in Figs. 6(b) and 6(d). The band $F_{11}$ [see Fig. 5(c)] lies in the surface mode sector. It derives from the frequency equation (C21). Close to the origin, the band has a quadratic asymptote:

$$
\omega_{F}(p) \simeq \frac{c_{h} R}{2} p^{2} .
$$


The density of states therefore diverges as $p \rightarrow 0$, which leads to a strong coupling between $F_{11}$ modes and trapped atoms, as we discuss in Sec. III. The normalized radial partial waves are

$$
\begin{aligned}
& \mathcal{W}_{\gamma}^{r}(r) \simeq \frac{1}{R}, \\
& \mathcal{W}_{\gamma}^{\varphi}(r) \simeq \frac{i j}{R}, \\
& \mathcal{W}_{\gamma}^{z}(r) \simeq-\frac{i p}{R} r
\end{aligned}
$$

to linear order in $p r$. Figures 6(c) and 6(e) show the displacement caused by an $\mathrm{F}_{11}$ mode.

As we discuss in Sec. III, there is experimental evidence that low-frequency $T_{01}$ modes are reflected at the tapered ends of the nanofiber, resulting in standing waves confined to the nanofiber region and discrete mechanical resonance frequencies. Likewise, we are interested in $F_{11}$ modes confined to the nanofiber as a way of reducing the atom heating. In order to obtain the corresponding phononic eigenmodes, it is in principle necessary to account for the two tapers that connect the nanofiber region to the regular macroscopic glass fiber and to solve the phononic eigenmode equation for this more complex geometry $[34,79]$. Here, we approximate the desired behavior by imposing periodic boundary conditions on the eigenmodes of an infinite cylinder and require the displacement to vanish at the beginning $(z=0)$ and the end of the nanofiber $(z=L)$. This condition can be met for all three fundamental modes when approximating the radial partial waves Eqs. (C32) and (C34) to constant order in $p r$, which is a good approximation for a nanofiber; compare Fig. 6. The resulting eigenmodes and corresponding strain modal fields are then of the form

$$
\begin{gathered}
\boldsymbol{w}_{\gamma}(\boldsymbol{r})=\frac{\mathcal{W}_{\gamma}(r)}{\sqrt{\pi L}} \sin \left(p_{m} z\right) e^{i j \varphi}, \\
\boldsymbol{s}_{\gamma}(\boldsymbol{r})=\frac{\mathcal{S}_{\gamma}(r)}{\sqrt{\pi L}} \cos \left(p_{m} z\right) e^{i j \varphi}
\end{gathered}
$$

instead of Eq. (C13), where $L$ is the length of the nanofiber. Torsional resonances in particular appear at $\omega_{m}=m \pi c_{t} / L$. The propagation constant can take only the discrete values

$$
p_{m}=m \pi / L, \quad m \in \mathbb{N},
$$

which form a subset of each fundamental band. The normalization condition Eq. (C14) for the radial partial waves is unchanged.

\section{APPENDIX D: ATOM-PHONON INTERACTION}

There are two mechanisms that lead to atom-phonon interaction, as we discuss in Sec. II of this article: dp and st. In consequence, the coupling functions $g_{\gamma}(\boldsymbol{r})$ appearing in the interaction Hamiltonian Eq. (6), as well as the coupling constants $g_{\gamma i}$ appearing in the linear-force interaction Hamiltonian Eq. (7) have two contributions:

$$
\begin{aligned}
g_{\gamma}(\boldsymbol{r}) & =g_{\gamma}^{\mathrm{dp}}(\boldsymbol{r})+g_{\gamma}^{\mathrm{st}}(\boldsymbol{r}), \\
g_{\gamma i} & =g_{\gamma i}^{\mathrm{dp}}+g_{\gamma i}^{\mathrm{st}} .
\end{aligned}
$$

In Appendixes D 1 and D 2, we model the dependence of the potential $V$ experienced by the atom on displacement $\boldsymbol{u}$ and strain $\boldsymbol{S}$ in the case of a two-color nanofiber-based atom trap. This model then enables us to derive explicit expressions for the coupling functions and the atomphonon coupling constants. Appendix D 3 provides additional details on how to calculate phonon-induced atom heating rates once the coupling constants are known.

\section{Displacement coupling}

Only modes on the three fundamental phonon bands $\mathrm{T}_{01}$, $\mathrm{L}_{01}$, and $\mathrm{F}_{11}$ of the nanofiber introduced in Appendix $\mathrm{C} 2$ have frequencies comparable to those of an atom moving in a nanofiber-based trap and will therefore interact significantly with the atom. Modes on the longitudinal $\mathrm{L}_{01}$ band and on the flexural $F_{11}$ band lead to a displacement of the surface at first order in $\boldsymbol{u}$. Modes on the torsional $\mathrm{T}_{01}$ band [Fig. 6(a)] lead to a change of the fiber radius of second order because $\boldsymbol{u}$ is orthogonal to the surface normal. In consequence, only the longitudinal and flexural modes will interact with the atom through displacement coupling in the linearized Hamiltonian Eq. (5).

The $\mathrm{L}_{01}$ modes [Fig. 6(b)] lead to a $z$-dependent modulation of the fiber radius by the radial displacement on the fiber surface $u^{r}(r=R, z)$, without displacing the fiber axis. The change in radius has two effects: First, it shifts the surface of the fiber together with the electromagnetic fields surrounding it relative to the trapped atom. Second, it leads to new photonic eigenmodes and therefore deforms the electromagnetic fields. As we discuss in Sec. II, we neglect the second effect and assume that both optical and surface potentials are shifted radially by $u^{r}(R, z) \boldsymbol{e}_{r}$ without being deformed.

The $\mathrm{F}_{11}$ modes [Fig. 6(c)] displace the entire fiber cross section in the plane orthogonal to the fiber axis by $u^{r}(R, \varphi, z) \boldsymbol{e}_{r}+u^{\varphi}(R, \varphi, z) \boldsymbol{e}_{\varphi}$ without changing the fiber radius. Since the wavelengths of the relevant vibrations are much larger than the optical wavelengths, the fiber appears approximately unchanged on length scales relevant for the photon modes. We can therefore again neglect deformations of the photon eigenmodes and model the effect of the flexural mode as a displacement of the entire potential along with the fiber cross section.

The effect of the fundamental modes is thus to shift the potential at position $\boldsymbol{r}$ by a vector $\Delta \boldsymbol{u}(\boldsymbol{r})$ that depends on the phonon field on the fiber surface. The direct dependence of the potential on the displacement can then be modeled as [67] 


$$
V[\boldsymbol{u}, \boldsymbol{S}](\boldsymbol{r}) \equiv V[\mathbf{0}, \boldsymbol{S}][\boldsymbol{r}-\Delta \boldsymbol{u}(\boldsymbol{r})] .
$$

The entire potential is shifted due to displacement of the fiber surface in addition to any changes to the potential that arise from the strain $S$ caused by displacement inside the fiber. This model allows us to evaluate the displacement coupling term in Eq. (5): The functional derivative reduces to conventional partial derivatives of the unperturbed potential $V_{0}$ and

$$
\delta_{\boldsymbol{u}} V_{(\mathbf{0}, \mathbf{0})}[\hat{\boldsymbol{u}}](\hat{\boldsymbol{r}})=-\Delta \hat{\boldsymbol{u}}(\hat{\boldsymbol{r}}) \cdot \nabla V_{0}(\hat{\boldsymbol{r}})
$$

By expanding the displacement field in terms of the fiber eigenmodes [see Eq. (C9)], the shifts due to the fundamental phonon modes can be summarized as

$$
\Delta \hat{\boldsymbol{u}}(\boldsymbol{r}) \equiv \sum_{\gamma} \mathcal{U}_{\gamma}\left\{\left[w_{\gamma}^{r}(R, \varphi, z) \boldsymbol{e}_{r}+\delta_{f F} w_{\gamma}^{\varphi}(R, \varphi, z) \boldsymbol{e}_{\varphi}\right] \hat{b}_{\gamma}+\text { H.c. }\right\} .
$$

Here, $\mathcal{U}_{\gamma}$ is the displacement mode density and $\boldsymbol{w}_{\gamma}$ the phonon eigenmodes Eq. (C13). The Kronecker symbol $\delta_{f F}$ selects the flexural mode family $f=\mathrm{F}$. This equation holds for all three fundamental bands since $w^{r}(\boldsymbol{r})=w^{\varphi}(\boldsymbol{r})=0$ for torsional modes. The resulting displacement coupling function in Eqs. (6) and (D1) is

$g_{\gamma}^{\mathrm{dp}}(\boldsymbol{r})=-\mathcal{U}_{\gamma}\left[w_{\gamma}^{r}(R, \varphi, z) \partial_{r} V_{0}(\boldsymbol{r})+\delta_{f F} w_{\gamma}^{\varphi}(R, \varphi, z) \frac{\partial_{\varphi}}{r} V_{0}(\boldsymbol{r})\right]$.

The corresponding displacement coupling constants for nanofiber-trapped atoms obtained from Eqs. (8) and (D5) are

$g_{\gamma r}^{\mathrm{dp}}=-\frac{\omega_{r}}{2}\left[\frac{\mathcal{U}_{\gamma} w_{0}^{r}}{\Delta r}+\delta_{f F}\left(\frac{\omega_{r \varphi}}{\omega_{r}}\right)^{2} \frac{\mathcal{U}_{\gamma} w_{0}^{\varphi}}{\Delta r}\right]$,

$g_{\gamma \varphi}^{\mathrm{dp}}=-\frac{\omega_{\varphi}}{2}\left[\delta_{f F} \frac{\mathcal{U}_{\gamma} w_{0}^{\varphi}}{r_{0} \Delta \varphi}+\left(\frac{\omega_{r \varphi}}{\omega_{\varphi}}\right)^{2} \frac{\mathcal{U}_{\gamma} w_{0}^{r}}{r_{0} \Delta \varphi}\right]$,

$g_{\gamma z}^{\mathrm{dp}}=-\frac{\omega_{z}}{2}\left[\left(\frac{\omega_{r z}}{\omega_{z}}\right)^{2} \frac{\mathcal{U}_{\gamma} w_{0}^{r}}{\Delta z}+\delta_{f F}\left(\frac{\omega_{\varphi z}}{\omega_{z}}\right)^{2} \frac{\mathcal{U}_{\gamma} w_{0}^{\varphi}}{\Delta z}\right]$

where

$$
w_{0}^{r} \equiv w_{\gamma}^{r}\left(R, \varphi_{0}, z_{0}\right), \quad w_{0}^{\varphi} \equiv w_{\gamma}^{\varphi}\left(R, \varphi_{0}, z_{0}\right)
$$

are the displacement modal fields evaluated on the fiber surface. Note that the model predicts only coupling between phonons and the axial motion of the atom if $\omega_{\varphi z} \neq 0$ or $\omega_{\varphi z} \neq 0$, that is, if the potential has symmetries misaligned with the cylindrical coordinate axes.

We can derive explicit expressions for the displacement coupling constants by using the approximate expressions for the displacement field of modes on the fundamental phonon bands $\mathrm{L}_{01}$ and $\mathrm{F}_{11}$ given in Appendix C 2. In case the cross-couplings $\omega_{i j}$ are negligible, the coupling constant of the radial atomic motion to an $\mathrm{L}_{01}$ phonon mode Eq. (32) of frequency $\omega_{\gamma}$ is

$$
\left|g_{L r}^{\mathrm{dp}}\right| \equiv \frac{\nu}{2 \pi c_{h}} \sqrt{\frac{M \omega_{r}^{3} \omega_{\gamma}}{2 \rho}},
$$

and there is no coupling to the azimuthal and axial motion $g_{L \varphi}^{\mathrm{dp}}=g_{L z}^{\mathrm{dp}}=0$. The coupling constant of the radial and azimuthal motion to an $\mathrm{F}_{11}$ phonon mode Eq. (C34) is

$$
\left|g_{F i}^{\mathrm{dp}}\right|=\frac{1}{4 \pi R} \sqrt{\frac{M \omega_{i}^{3}}{\rho \omega_{\gamma}}}, \quad i \in\{r, \varphi\},
$$

and there is no coupling to the axial motion $g_{F z}^{\mathrm{dp}}=0$.

The model Eq. (D2) relies on the simple geometrical shape of the nanofiber and the symmetries of its fundamental mechanical modes. Nanophotonic structures that have more complex geometries in general require a more careful analysis of the change of optical and dispersion potentials. The variation of the optical potential, for instance, can be modeled more generally by perturbatively calculating the new photonic eigenmodes in the presence of shifted boundaries of the nanostructure [66]. We choose a similar approach in the next section to obtain the perturbed eigenmodes in the presence of a modified permittivity but unchanged boundaries.

\section{Strain coupling}

All three fundamental phonon bands $\mathrm{T}_{01}, \mathrm{~L}_{01}$, and $\mathrm{F}_{11}$ of the nanofiber induce strain in the fiber. In order to evaluate the strain coupling term $\delta V_{(\mathbf{0}, \mathbf{0})}[\boldsymbol{S}]$ in Eq. (5), we model how each phonon mode changes the potential through the strain it causes. We neglect the influence of strain on the surface forces $\delta_{S} V_{\text {ad }(\mathbf{0}, \mathbf{0})}[\boldsymbol{S}]=0$, as we discuss in Sec. II. The strain dependence then arises only from changes of the red- and blue-detuned optical potentials. A nonzero strain changes the electromagnetic properties of the fiber due to the photoelastic effect, which we model through a straindependent permittivity tensor $\overline{\boldsymbol{\epsilon}}[\boldsymbol{S}][34,68,69,78]$. A modified permittivity leads to new photonic eigenmodes and electric modal fields $\overline{\boldsymbol{e}}_{\eta}[\overline{\boldsymbol{\epsilon}}]$, and therefore, to modified electric fields $\overline{\boldsymbol{E}}_{0}\left[\left\{\overline{\boldsymbol{e}}_{\eta}\right\}\right]$ surrounding the fiber. In consequence, the optical potential $V_{\text {opt }}^{r}\left[\overline{\boldsymbol{E}}_{0}^{r}\right]+V_{\text {opt }}^{b}\left[\overline{\boldsymbol{E}}_{0}^{b}\right]$ created by the red- and blue-detuned light field is changed, and the potential $V[\boldsymbol{u}, \boldsymbol{S}]$ ultimately depends on strain.

The photoelastic effect can be quantified by a tensor $\boldsymbol{P}$ of fourth rank called the photoelastic tensor, which phenomenologically describes how the optical properties of a material change under strain [68]: 


$$
\left(\overline{\boldsymbol{\epsilon}}^{-1}\right)^{i j}[\boldsymbol{S}]=\left[\left(\boldsymbol{\epsilon}^{-1}\right)^{i j}+\sum_{k l} P^{i j k l} S^{k l}\right],
$$

where the exponent $(-1)$ indicates the inverse tensor. The photoelastic tensor has symmetries $P^{i j k l}=P^{j i k l}=P^{i j l k}$ and therefore possesses at most 36 independent components [68]. We use a compact index notation to group the first pair of indices $i j \equiv(N)$ and the second pair of indices $k l \equiv(M)$ according to $11 \equiv(1), 22 \equiv(2), 33 \equiv(3)$, $12,21 \equiv(4), 13,31 \equiv(5)$, and $23,32 \equiv(6)$. The independent components can then be arranged in a $6 \times 6$ matrix $(\boldsymbol{P})$, where $N$ corresponds to the row and $M$ to the column number. For materials like silica that exhibit a homogeneous and isotropic photoelastic effect, the components of the photoelasticity tensor $\boldsymbol{P}$ in both Cartesian and cylindrical coordinates are [112]

$$
(\boldsymbol{P})=\left(\begin{array}{cccccc}
P_{1} & P_{2} & P_{2} & 0 & 0 & 0 \\
P_{2} & P_{1} & P_{2} & 0 & 0 & 0 \\
P_{2} & P_{2} & P_{1} & 0 & 0 & 0 \\
0 & 0 & 0 & P_{3} & 0 & 0 \\
0 & 0 & 0 & 0 & P_{3} & 0 \\
0 & 0 & 0 & 0 & 0 & P_{3}
\end{array}\right),
$$

where $P_{1}, P_{2} \in \mathbb{R}$ and $P_{3}=\left(P_{1}-P_{2}\right) / 2$.

We are interested in the strain-induced variation $\Delta \epsilon \equiv$ $\overline{\boldsymbol{\epsilon}}[\boldsymbol{S}]-\boldsymbol{\epsilon}$ of the permittivity tensor. To linear order in the strain,

$$
\Delta \epsilon^{i j} \simeq\left(D \bar{\epsilon}^{i j}\right)_{\mathbf{0}}[\boldsymbol{S}]=-\epsilon^{2} \sum_{k l} P^{i j k l} S^{k l}
$$

for a medium that is isotropic while unperturbed $\epsilon=\epsilon \mathbb{1}$.

The new photonic eigenmodes $\overline{\boldsymbol{a}}[\overline{\boldsymbol{\epsilon}}]$ in the presence of a modified permittivity $\overline{\boldsymbol{\epsilon}}$ are solutions to the photonic eigenmode equation

$$
\mathcal{D} \overline{\boldsymbol{a}}_{\eta}=-\bar{d}_{\eta} \overline{\boldsymbol{\epsilon}} \overline{\boldsymbol{a}}_{\eta} .
$$

Compare Eq. (A3), where $\bar{d}_{\eta}=\bar{\omega}_{\eta}^{2} / c^{2}$ and $\bar{\omega}_{\eta}$ are the frequencies of the perturbed eigenmodes. We are interested in the new eigenmodes $\overline{\boldsymbol{a}}_{\eta}$ and eigenvalues $\bar{d}_{\eta}$ in the presence of a perturbation $\Delta \boldsymbol{\epsilon}$ of the permittivity tensor. To this end, we perturbatively expand both eigenmodes and eigenvalues in orders $n$ of $\Delta \boldsymbol{\epsilon}$, analogous to time-independent perturbation theory in quantum mechanics [113]:

$$
\begin{array}{ll}
\bar{d}_{\eta} \equiv d_{\eta}+\Delta d_{\eta}, & \Delta d_{\eta} \equiv \sum_{n} d_{\eta}^{(n)}, \\
\overline{\boldsymbol{a}}_{\eta} \equiv c \boldsymbol{a}_{\eta}+\Delta \boldsymbol{a}_{\eta}, & \Delta \boldsymbol{a}_{\eta} \equiv \sum_{n} \boldsymbol{a}_{\eta}^{(n)} .
\end{array}
$$

The normalization constant $c \in \mathbb{C}$ is found by normalizing the perturbed eigenmode $\overline{\boldsymbol{a}}_{\eta}$. This expansion, in conjunction with Eq. (D13) and the orthogonality relation Eq. (A4), leads to the relations

$$
\Delta d_{\eta}=-d_{\eta} \frac{\left(\boldsymbol{a}_{\eta} \mid \Delta \boldsymbol{\epsilon} \overline{\boldsymbol{a}}_{\eta}\right)}{\left(\boldsymbol{a}_{\eta} \mid(\boldsymbol{\epsilon}+\Delta \boldsymbol{\epsilon}) \overline{\boldsymbol{a}}_{\eta}\right)}
$$

and

$$
\begin{aligned}
\Delta \boldsymbol{a}_{\eta}= & \sum_{\eta^{\prime} \neq \eta} \frac{\boldsymbol{\epsilon}^{-1}}{d_{\eta^{\prime}}-d_{\eta}}\left[d_{\eta}\left(\boldsymbol{a}_{\eta^{\prime}} \mid \boldsymbol{\epsilon} \Delta \boldsymbol{\epsilon} \overline{\boldsymbol{a}}_{\eta}\right)\right. \\
& \left.+\Delta d_{\eta}\left(\boldsymbol{a}_{\eta^{\prime}} \mid \boldsymbol{\epsilon}(\boldsymbol{\epsilon}+\Delta \boldsymbol{\epsilon}) \overline{\boldsymbol{a}}_{\eta}\right)\right] \boldsymbol{a}_{\eta^{\prime}}
\end{aligned}
$$

for the corrections to eigenvalues and eigenmodes. The bracket indicates the $L^{2}$ scalar product,

$$
(\boldsymbol{A} \mid \boldsymbol{b})=\int \boldsymbol{A}^{*} \cdot \boldsymbol{b} d \boldsymbol{r}
$$

Equation (D17) holds provided the perturbed eigenmode $\overline{\boldsymbol{a}}_{\eta}$ does not overlap with modes $\boldsymbol{a}_{\eta^{\prime}}$ degenerate with the unperturbed mode $\boldsymbol{a}_{\eta}$, that is, $d_{\eta^{\prime}}=d_{\eta}$ only if $\left(\boldsymbol{a}_{\eta^{\prime}} \mid \boldsymbol{\epsilon} \Delta \boldsymbol{\epsilon} \overline{\boldsymbol{a}}_{\eta}\right)=0=\left(\boldsymbol{a}_{\eta^{\prime}} \mid \boldsymbol{\epsilon}(\boldsymbol{\epsilon}+\Delta \boldsymbol{\epsilon}) \overline{\boldsymbol{a}}_{\eta}\right)$. The corrections $\Delta d_{\eta}$ and $\Delta \boldsymbol{a}_{\eta}$ can be obtained order by order in $\Delta \boldsymbol{\epsilon}$. The firstorder correction to the eigenvalue is

$$
d_{\eta}^{(1)}=-d_{\eta} \frac{\left(\boldsymbol{a}_{\eta} \mid \Delta \boldsymbol{\epsilon} \boldsymbol{a}_{\eta}\right)}{\left(\boldsymbol{a}_{\eta} \mid \boldsymbol{\epsilon} \boldsymbol{a}_{\eta}\right)}
$$

which reduces to the known formula for first-order corrections of the eigenfrequency in case of isotropic permittivities $\boldsymbol{\epsilon}, \overline{\boldsymbol{\epsilon}}$ [86]. The first-order correction to the eigenmode is

$\boldsymbol{a}_{\eta}^{(1)}=\sum_{\eta^{\prime} \neq \eta} \frac{\boldsymbol{\epsilon}^{-1}}{d_{\eta^{\prime}}-d_{\eta}}\left[d_{\eta}\left(\boldsymbol{a}_{\eta^{\prime}} \mid \boldsymbol{\epsilon} \Delta \boldsymbol{\epsilon} \boldsymbol{a}_{\eta}\right)-d_{\eta}^{(1)}\left(\boldsymbol{a}_{\eta^{\prime}} \mid \boldsymbol{\epsilon}^{2} \boldsymbol{a}_{\eta}\right)\right] \boldsymbol{a}_{\eta^{\prime}}$,

where we use that $c=1+\mathcal{O}(\Delta \boldsymbol{\epsilon})$ [113].

In the case study in Sec. III of this article, we are concerned with the case $\boldsymbol{\epsilon}=\epsilon \mathbb{1}$. The first-order correction to the eigenvalue then simplifies to

$$
d_{\eta}^{(1)}=-d_{\eta} \frac{\int \boldsymbol{a}_{\eta}^{*} \cdot\left(\Delta \boldsymbol{\epsilon} \boldsymbol{a}_{\eta}\right) d \boldsymbol{r}}{\int \boldsymbol{a}_{\eta}^{*} \cdot \boldsymbol{a}_{\eta} d \boldsymbol{r}}
$$

Moreover, one can show that the first-order shift is zero for perturbations of the permittivity caused by nanofiber phonons of propagation constant $p \neq 0$, so 


$$
\boldsymbol{a}_{\eta}^{(1)}=\sum_{\eta^{\prime} \neq \eta} \boldsymbol{a}_{\eta^{\prime}} \frac{d_{\eta}}{d_{\eta^{\prime}}-d_{\eta}} \int \boldsymbol{a}_{\eta^{\prime}}^{*} \cdot\left(\Delta \boldsymbol{\epsilon} \boldsymbol{a}_{\eta}\right) d \boldsymbol{r} .
$$

Consider, for example, a mode on the $\mathrm{HE}_{11}$ band of a fiber; see Fig. 3(b). Fiber phonon modes with azimuthal order $j=0$ lead to the population of photon modes on the same band, at slightly different propagation constants $k$. Phonon modes with azimuthal order $j= \pm 1$, on the other hand, can populate modes on the $\mathrm{TE}_{01}, \mathrm{TM}_{01}$, and $\mathrm{HE}_{21}$ bands shown Figs. 3(a) and 3(c). We neglect coupling to radiative modes (leading to phonon-induced transmission losses), since radiative fields are extended, with low amplitudes, and interact only very weakly with the atom.

Having obtained the first-order correction to the photonic eigenmodes, we can now approximate variation $\Delta \boldsymbol{e}_{\eta}$ of the electric modal field due to the modified permittivity. Using Eq. (A6), $\Delta \boldsymbol{e}_{\eta} \simeq i\left(D \overline{\boldsymbol{a}}_{\eta}\right)_{\boldsymbol{\epsilon}}[\Delta \boldsymbol{\epsilon}] / \epsilon_{0}=i \boldsymbol{a}_{\eta}^{(1)} / \epsilon_{0}$ to linear order in the permittivity. The variation of the electric modal field is thus,

$$
\Delta \boldsymbol{e}_{\eta} \simeq \sum_{\eta^{\prime} \neq \eta} \boldsymbol{e}_{\eta^{\prime}} \frac{\omega_{\eta}^{2}}{\omega_{\eta^{\prime}}^{2}-\omega_{\eta}^{2}} \int \boldsymbol{a}_{\eta^{\prime}} \cdot\left(\Delta \boldsymbol{\epsilon} \boldsymbol{a}_{\eta}\right) d \boldsymbol{r}
$$

for nanofiber eigenmodes.

The light coupled into the fiber determines the frequencies at which photonic modes are populated. Since there are no frequency shifts of the eigenmodes at first order in $\Delta \boldsymbol{\epsilon}$, we can assume that the amplitude $\alpha_{\eta}$ of each photonic mode remains unchanged, while its spatial form $\overline{\boldsymbol{e}}_{\eta}$ is periodically modified by the vibrations. The modified complex field profile of a monochromatic light field is therefore,

$$
\overline{\boldsymbol{E}}_{0}\left[\left\{\overline{\boldsymbol{e}}_{\eta}\right\}\right] \simeq \sum_{\eta} \alpha_{\eta} \overline{\boldsymbol{e}}_{\eta}
$$

To linear order in the modal fields, the variation of the field profile $\Delta \boldsymbol{E}_{0} \equiv \overline{\boldsymbol{E}}_{0}-\boldsymbol{E}_{0}$ is

$$
\Delta \boldsymbol{E}_{0} \simeq \sum_{\eta}\left(\delta_{\overline{\boldsymbol{e}}_{\eta}} \overline{\boldsymbol{E}}_{0}\right)_{\boldsymbol{e}_{\eta}}\left[\left\{\Delta \boldsymbol{e}_{\eta}\right\}\right]=\sum_{\eta} \alpha_{\eta} \Delta \boldsymbol{e}_{\eta} .
$$

The changed electric fields lead to a changed optical potential $V_{\text {opt }}^{r}\left[\overline{\boldsymbol{E}}_{0}^{r}\right]+V_{\text {opt }}^{b}\left[\overline{\boldsymbol{E}}_{0}^{b}\right]$. We can now use the chain rule to express the strain coupling term in the interaction Hamiltonian Eq. (5) through the derivative of the optical potential with respect to the electric fields [114]:

$$
\delta_{S} V_{(\mathbf{0 , 0})}[\boldsymbol{S}]=\left(D V_{\mathrm{opt}}^{r}\right)_{\boldsymbol{E}_{0}^{r}}\left[\boldsymbol{\Delta} \boldsymbol{E}_{0}^{r}\right]+\left(D V_{\mathrm{opt}}^{b}\right)_{\boldsymbol{E}_{0}^{b}}\left[\boldsymbol{\Delta} \boldsymbol{E}_{0}^{b}\right] .
$$

Each optical potential is the sum of scalar, vector, and tensor light shift; see Eq. (B4). Thus,

$$
\delta_{S} V_{(\mathbf{0}, \mathbf{0})}[\boldsymbol{S}]=\sum_{j}\left(D V_{j}^{r}\right)_{\boldsymbol{E}_{0}^{r}}\left[\Delta \boldsymbol{E}_{0}^{r}\right]+\left(D V_{j}^{b}\right)_{\boldsymbol{E}_{0}^{b}}\left[\Delta \boldsymbol{E}_{0}^{b}\right]
$$

where $j \in\{s, v, t\}$ for the scalar, vector, and tensor contributions given in Eqs. (B5), (B6), and (B8). The functional derivatives of the light shifts reduce to conventional derivatives. For each of the two colors, the derivative of the scalar light shift is

$$
\left(D V_{s}\right)_{\boldsymbol{E}_{0}}\left[\Delta \boldsymbol{E}_{0}\right]=-\alpha_{s}\left[\boldsymbol{E}_{0}^{*}(\boldsymbol{r}) \cdot \Delta \boldsymbol{E}_{0}(\boldsymbol{r})+\text { c.c. }\right],
$$

the derivative of the vector light shift

$$
\left(D V_{v}\right)_{\boldsymbol{E}_{0}}\left[\Delta \boldsymbol{E}_{0}\right]=-\frac{\alpha_{v}}{2 i} \frac{M_{F}}{F}\left[\boldsymbol{E}_{0}^{*}(\boldsymbol{r}) \times \Delta \boldsymbol{E}_{0}(\boldsymbol{r})-\text { c.c. }\right] \cdot z_{B},
$$

and the derivative of the tensor light shift

$$
\begin{aligned}
\left(D V_{t}\right)_{\boldsymbol{E}_{0}}\left[\Delta \boldsymbol{E}_{0}\right]= & -3 \alpha_{t} \frac{3 M_{F}^{2}-F(F+1)}{2 F(2 F-1)} \\
& \times\left[E_{0}^{* z_{B}}(\boldsymbol{r}) \Delta E_{0}^{z_{B}}(\boldsymbol{r})+\text { c.c. }\right] .
\end{aligned}
$$

Finally, the strain coupling functions $g_{\gamma}^{\text {st }}(\boldsymbol{r})$ are obtained by making the dependence on strain explicit in Eqs. (D28)(D30) and by expanding the strain operator Eq. (C12) in terms of the strain modal fields $\boldsymbol{s}_{\gamma}$ of a nanofiber. The strain coupling function then has contributions from the three light shifts $j \in\{s, v, t\}$ for both light colors $\{r, b\}$ :

$$
g_{\gamma}^{\mathrm{st}}(\boldsymbol{r})=\sum_{j}\left[g_{\gamma}^{r j}(\boldsymbol{r})+g_{\gamma}^{b j}(\boldsymbol{r})\right] .
$$

The contributions of the three light shifts to the coupling functions are listed in Table XVI for a monochromatic light field. Note that the difference between running waves Eq. (C13) and standing waves Eq. (C35) leads to different coupling functions for discrete modes on the $\mathrm{T}_{01}$ band on one hand and modes on the continuous $\mathrm{L}_{01}$ and $\mathrm{F}_{11}$ bands on the other hand. The corresponding strain coupling constants $g_{\gamma i}^{\mathrm{st}}$ are obtained using the definition Eq. (8).

To conclude the derivation of the strain coupling, let us illustrate the strain-induced change of the potential due to the photoelastic effect. To first order, the change is given by the strain coupling term $\delta_{S} V_{(\mathbf{0 , 0})}[\hat{\boldsymbol{S}}](\boldsymbol{r})$. We consider the nanofiber to undergo macroscopic vibrations described by the multimode coherent state $|\beta\rangle$ with amplitudes $\beta_{\gamma}=$ $\left|\beta_{\gamma}\right| e^{i \phi_{\gamma}} \in \mathbb{C}$ for each mode. The expectation value of the change caused by strain is then

$$
\begin{aligned}
& \left\langle\beta, t\left|\delta_{S} V_{(\mathbf{0}, \mathbf{0})}[\hat{\boldsymbol{S}}](\boldsymbol{r})\right| \beta, t\right\rangle \\
& =2 \sum_{\gamma}\left|\beta_{\gamma}\right|\left[\cos \left(\omega_{\gamma} t-\phi_{\gamma}\right) \operatorname{Re} g_{\gamma}^{\mathrm{st}}(\boldsymbol{r})-\sin \left(\omega_{\gamma} t-\phi_{\gamma}\right) \operatorname{Im} g_{\gamma}^{\mathrm{st}}(\boldsymbol{r})\right] .
\end{aligned}
$$

The coupling function $g_{\gamma}^{\text {st }}(\boldsymbol{r})$ therefore describes the change to the potential $V$ due to a phonon mode $\gamma$. We 
TABLE XVI. Atom-phonon coupling functions due to strain in a nanofiber-based atom trap. The coupling functions correspond to scalar, vector, and tensor light shift induced by a single monochromatic light field of frequency $\omega_{0}$ and complex field profile $\boldsymbol{E}_{0}$. All symbols are defined in Appendixes A, C, and D. In particular, the photon mode indices are $\eta=(m, f, k, n)$ as defined in Eq. (A15), and the phonon mode indices $\gamma=(j, f, p, n)$ as defined in Eq. (C27). The index $\eta$ designates an unperturbed eigenmode, while the primed index $\eta^{\prime}$ labels modes perturbatively populated due to strain.

$$
\begin{aligned}
& \text { Continuous phonon modes: } f=\mathrm{L}, \mathrm{F} \\
& g_{\gamma}^{s}(\boldsymbol{r})=-\alpha_{s}\left[\boldsymbol{E}_{0}^{*}(\boldsymbol{r}) \cdot \Delta \boldsymbol{E}_{\gamma}^{-}(\boldsymbol{r})+\boldsymbol{E}_{0}(\boldsymbol{r}) \cdot \Delta \boldsymbol{E}_{\gamma}^{+*}(\boldsymbol{r})\right] \\
& g_{\gamma}^{v}(\boldsymbol{r})=-\left(\alpha_{v} / 2 i\right)\left(M_{F} / F\right)\left[\boldsymbol{E}_{0}^{*}(\boldsymbol{r}) \times \Delta \boldsymbol{E}_{\gamma}^{-}(\boldsymbol{r})-\boldsymbol{E}_{0}(\boldsymbol{r}) \times \Delta \boldsymbol{E}_{\gamma}^{+*}(\boldsymbol{r})\right] \cdot z_{B} \\
& g_{\gamma}^{t}(\boldsymbol{r})=-3 \alpha_{t}\left\{\left[3 M_{F}^{2}-F(F-1)\right] /[2 F(2 F+1)]\right\}\left[E_{0}^{* z_{B}}(\boldsymbol{r}) \Delta E_{\gamma}^{-z_{B}}(\boldsymbol{r})+E_{0}^{z_{B}}(\boldsymbol{r}) \Delta E_{\gamma}^{+* z_{B}}(\boldsymbol{r})\right] \\
& \Delta \boldsymbol{E}_{\gamma}^{ \pm}(\boldsymbol{r})=-\left.\sum_{\eta f^{\prime} n^{\prime}} \alpha_{\eta} \mathcal{A}_{\eta^{\prime} \gamma \eta}^{ \pm} \boldsymbol{e}_{\eta^{\prime}}(\boldsymbol{r})\right|_{\substack{m^{\prime} \prime m \pm j \\
k^{\prime}=k \pm p}} \\
& \mathcal{A}_{\eta^{\prime} \gamma \eta}^{-}=\left[\omega_{0}^{2} /\left(\omega_{\eta^{\prime}}^{2}-\omega_{0}^{2}\right)\right]\left[\mathcal{U}_{\gamma} /(2 \pi)\right] \epsilon^{2} \int_{0}^{R}\left[r \boldsymbol{r}_{\eta^{\prime}}^{*}(r) \boldsymbol{P} \mathcal{S}_{\gamma}(r) \boldsymbol{a}_{\eta}(r)\right] d r \\
& \mathcal{A}_{\eta^{\prime} \gamma \eta}^{+}=\left[\omega_{0}^{2} /\left(\omega_{\eta^{\prime}}^{2}-\omega_{0}^{2}\right)\right]\left[\mathcal{U}_{\gamma} /(2 \pi)\right] \epsilon^{2} \int_{0}^{R}\left[r \boldsymbol{a}_{\eta^{\prime}}^{*}(r) \boldsymbol{P} \mathcal{S}_{\gamma}^{*}(r) \boldsymbol{a}_{\eta}(r)\right] d r
\end{aligned}
$$

Discrete phonon modes: $f=\mathrm{T}$

$$
\begin{aligned}
& g_{\gamma}^{s}(\boldsymbol{r})=-2 \alpha_{s} \operatorname{Re}\left[\boldsymbol{E}_{0}^{*}(\boldsymbol{r}) \cdot \Delta \boldsymbol{E}_{\gamma}(\boldsymbol{r})\right] \\
& g_{\gamma}^{v}(\boldsymbol{r})=-\alpha_{v}\left(M_{F} / F\right) \operatorname{Im}\left[\boldsymbol{E}_{0}^{*}(\boldsymbol{r}) \times \Delta \boldsymbol{E}_{\gamma}(\boldsymbol{r})\right] \cdot \boldsymbol{z}_{B} \\
& g_{\gamma}^{t}(\boldsymbol{r})=-6 \alpha_{t}\left\{\left[3 M_{F}^{2}-F(F-1)\right] /[2 F(2 F+1)]\right\} \operatorname{Re}\left[E_{0}^{* z_{B}}(\boldsymbol{r}) \Delta E_{\gamma}^{z_{B}}(\boldsymbol{r})\right] \\
& \Delta \boldsymbol{E}_{\gamma}(\boldsymbol{r})=\frac{1}{2}\left[\Delta \boldsymbol{E}_{\gamma}^{-}(\boldsymbol{r})+\Delta \boldsymbol{E}_{\gamma}^{+}(\boldsymbol{r})\right] \\
& \Delta \boldsymbol{E}_{\gamma}^{ \pm}(\boldsymbol{r})=-\left.\sum_{\eta f^{\prime} n^{\prime}} \alpha_{\eta} \mathcal{A}_{\eta^{\prime} \gamma \eta} \boldsymbol{e}_{\eta^{\prime}}(\boldsymbol{r})\right|_{\substack{m^{\prime}=m \pm j \\
k^{\prime}=m \pm p}} \\
& \mathcal{A}_{\eta^{\prime} \gamma \eta}=\left[\omega_{0}^{2} /\left(\omega_{\eta^{\prime}}^{2}-\omega_{0}^{2}\right)\right]\left[\mathcal{U}_{\gamma} /\left(\sqrt{k^{\prime}=k \pm p}\right)\right] \epsilon^{2} \int_{0}^{R}\left[r \boldsymbol{a}_{\eta^{\prime}}^{*}(r) \boldsymbol{P} \mathcal{S}_{\gamma}(r) \boldsymbol{a}_{\eta}(r)\right] d r \\
& \boldsymbol{a}_{\eta^{\prime}}^{*} \boldsymbol{P} \mathcal{S}_{\gamma} \boldsymbol{a}_{\eta}=a_{\eta}^{r} a_{\eta^{\prime}}^{r *}\left[P_{1} \mathcal{S}_{\gamma}^{r r}+P_{2}\left(\mathcal{S}_{\gamma}^{\varphi \varphi}+\mathcal{S}_{\gamma}^{z z}\right)\right]+a_{\eta}^{\varphi} a_{\eta^{\prime}}^{\varphi *}\left[P_{1} \mathcal{S}_{\gamma}^{\varphi \varphi}+P_{2}\left(\mathcal{S}_{\gamma}^{r r}+\mathcal{S}_{\gamma}^{z z}\right)\right]+a_{\eta}^{z} a_{\eta^{\prime}}^{z *}\left[P_{1} \mathcal{S}_{\gamma}^{z z}+P_{2}\left(\mathcal{S}_{\gamma}^{r r}+\mathcal{S}_{\gamma}^{\varphi \varphi}\right)\right] \\
& +\left(a_{\eta}^{r} a_{\eta^{\prime}}^{\varphi *}+a_{\eta}^{\varphi} a_{\eta^{\prime}}^{r *}\right)\left(P_{1}-P_{2}\right) \mathcal{S}_{\gamma}^{r \varphi}+\left(a_{\eta}^{r} a_{\eta^{\prime}}^{z *}+a_{\eta}^{z} a_{\eta^{\prime}}^{r *}\right)\left(P_{1}-P_{2}\right) \mathcal{S}_{\gamma}^{r z}+\left(a_{\eta}^{\varphi \eta} a_{\eta^{\prime}}^{z *}+a_{\eta}^{z} a_{\eta^{\prime}}^{\varphi * *}\right)\left(P_{1}-P_{2}\right) \mathcal{S}_{\gamma}^{\varphi z}
\end{aligned}
$$

plot $V_{0}(\boldsymbol{r})+2 \beta_{\gamma} \operatorname{Re} g_{\gamma}^{\mathrm{st}}(\boldsymbol{r})$ in Fig. 7 as an example of how strain due to a single torsional mode $\gamma$ perturbs the potential. The torsional mode qualitatively leads to rotation of the potential around the fiber axis, which results in a coupling between the torsional mode and the atom motion in the azimuthal and radial direction.

\section{Atom heating}

Let us consider an atom trapped in the optical near field of a nanofiber of temperature $T$. We assume that the atom is in the motional pure quantum ground state $\hat{\mu}_{0}$ of the harmonic trap at time $t=0$. At the same time, the phonon field of the fiber is in the thermal quantum state $\hat{\sigma}_{\mathrm{th}} \equiv$ $\exp \left(-\hat{H}_{\mathrm{phn}} / k_{B} T\right) / \operatorname{tr}\left[\exp \left(-\hat{H}_{\mathrm{phn}} / k_{B} T\right)\right]$ [72]. Over time, the atom acquires energy by absorbing phonons from the fiber, reflected in the increase of the expected number of motional quanta (population) $n_{i}(t) \equiv \operatorname{tr}\left[\hat{\rho}(t) \hat{a}_{i}^{\dagger} \hat{a}_{i}\right]$ along the spatial direction $i \in\{r, \varphi, z\}$. Here, $\hat{\rho}(t)$ is the state operator of the coupled atom-phonon system at times $t$, and $\operatorname{tr}$ is the trace. The population is initially zero. The evolution of $\hat{\rho}(t)$ from the initial state $\hat{\rho}_{0}=\hat{\mu}_{0} \otimes \hat{\sigma}_{\mathrm{th}}$ is governed by the full Hamiltonian Eq. (1). Provided the atom-phonon coupling is weak $g_{\gamma i} \ll \omega_{i}, \omega_{\gamma}$, the population grows linearly for sufficiently short times $t>0$, with the phonon-induced ground-state heating rate $\Gamma_{i}^{\text {th }}$ in trap

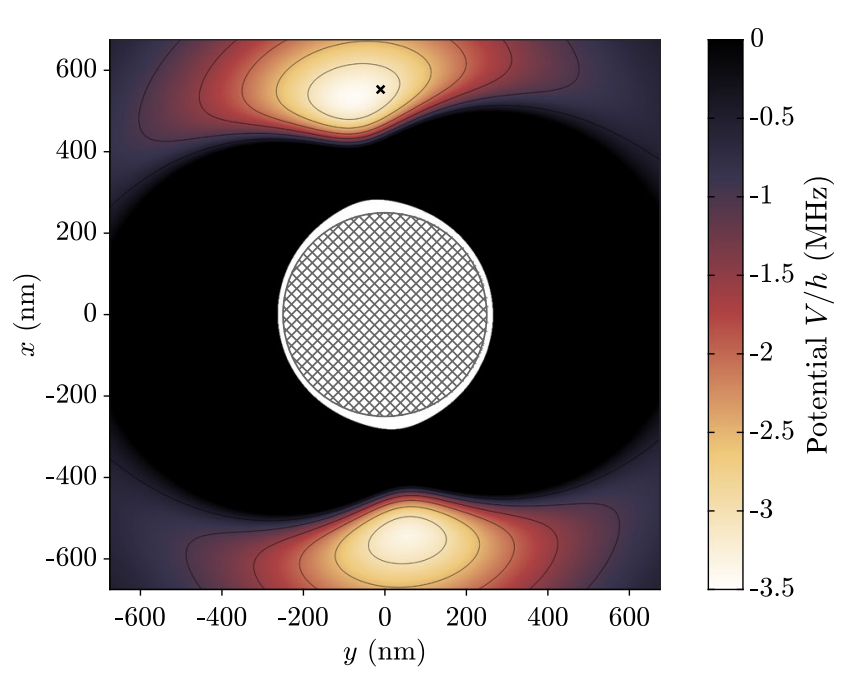

FIG. 7. Potential $V_{0}(\boldsymbol{r})+2 \beta_{\gamma} \operatorname{Re} g_{\gamma}^{\text {st }}(\boldsymbol{r})$ perturbed by strain due to the single, coherently excited torsional mode $\gamma$ of a nanofiber. The potential is evaluated at the trap minimum $z_{0}$ close to the center of the nanofiber. The mode $\gamma$ is the discrete torsional mode closest to resonance with the atom trap in the azimuthal direction; see Appendix E for the parameters. The amplitude $\beta_{\gamma}$ of the coherent excitation is exaggerated to an unphysical value such that the effect is visible at the given scales. It is apparent that the torsional mode couples to the atomic motion both in the radial and azimuthal direction. However, the contribution to the atom heating rate turns out to be negligible; see Sec. III. 
direction $i$. As we discuss in Sec. II, we distinguish the contributions of continuous phonon bands and discrete mechanical resonances to the atom heating rate,

$$
\Gamma_{i}^{\mathrm{th}}=\Gamma_{i}^{c}+\Gamma_{i}^{d} .
$$

Fermi's golden rule Eq. (11) can be used to calculate the contribution of the continuous phonon bands. For a nanofiber, there are only two continuous phonon bands resonant with the trapped atom: the longitudinal $\mathrm{L}_{01}$ band and the flexural $\mathrm{F}_{11}$ band. In consequence,

$$
\Gamma_{i}^{c}=\Gamma_{L i}+\Gamma_{F i} .
$$

The $\mathrm{L}_{01}$ band has dispersion relation $\omega_{\gamma}=c_{h}|p|$ in the lowfrequency limit [see Eq. (30)], resulting in a constant density of state $\rho_{L} \equiv 1 / c_{h}$. There are two resonant longitudinal modes $\gamma_{\sigma_{z}}$ at each trap frequency $\omega_{i}$, with propagation constants $p=\sigma_{z} \omega_{i} / c_{h}$ and $\sigma_{z}= \pm$. The contribution of longitudinal phonon modes to the ground-state heating rate is thus,

$$
\Gamma_{L i}=\frac{2 \pi \bar{n}_{i}}{c_{h}} \sum_{\sigma_{z}}\left|g_{\gamma_{\sigma_{z}}}\right|^{2}
$$

The $\mathrm{F}_{11}$ band has dispersion relation $\omega_{\gamma}=c_{h} R p^{2} / 2$ in the low-frequency limit [see Eq. (C33)], resulting in the density of states $\rho_{F i} \equiv 1 / \sqrt{2 \omega_{i} c_{h} R}$. There are four resonant flexural modes propagating in direction $\sigma_{z}= \pm$ and of $\sigma_{\varphi}= \pm$ circular polarization. The corresponding azimuthal order is $j=\sigma_{\varphi} \sigma_{z}$, and the propagation constant $p=\sigma_{z} \sqrt{2 \omega_{i} / c_{h} R}$. The ground-state heating rate due to the fundamental flexural phonon modes then simplifies to

$$
\Gamma_{F i}=\frac{2 \pi \bar{n}_{i}}{\sqrt{2 \omega_{i} c_{h} R}} \sum_{\sigma_{\varphi}, \sigma_{z}}\left|g_{\gamma_{\sigma_{\varphi} \sigma_{z}}}\right|^{2} .
$$

The contribution of each mode $\gamma_{i}$ on the continuous phonon bands to the heating rate Eq. (11) is proportional to the density of states $\rho_{\gamma_{i}}$. Since the $\mathrm{F}_{11}$ band is asymptotically quadratic [see Fig. 5(c)], the density of states of the flexural modes diverges as $\omega_{i} \rightarrow 0$. This dependence is reflected by $\Gamma_{F i} \propto 1 / \sqrt{\omega_{i}}$ in Eq. (D36). On the other hand, the density of states of the longitudinal modes is constant because the $\mathrm{L}_{01}$ has a linear asymptote in the low-frequency limit; see Fig. 5(b). The effect of flexural modes is therefore enhanced in comparison with longitudinal modes for atom-trap frequencies $\omega_{i}$ that are small compared to the frequency scale of the phonon bands. Moreover, the contribution of strain coupling is negligible for flexural modes. Using the displacement coupling constants Eq. (D9) in Eq. (D36) yields the formula Eq. (15) for the atom heating rate due to flexural modes, which is sufficient to explain heating rates observed in experiments; see Sec. III.
The discrete torsional modes are not reflected perfectly at the end of the nanofiber and therefore have a finite lifetime corresponding to decay rates $\kappa_{\gamma}$. This behavior can be modeled by including dissipation in the dynamics of the phonon field. The evolution of the density matrix $\hat{\rho}$ of the coupled atom-phonon system is then governed by the master equation [71]

$$
\frac{d}{d t} \hat{\rho}(t)=\frac{1}{i \hbar}[\hat{H}, \hat{\rho}]+\sum_{\gamma} \kappa_{\gamma}\left(\bar{n}_{\gamma}+1\right) \mathcal{D}_{\hat{b}_{\gamma}}(\hat{\rho})+\sum_{\gamma} \kappa_{\gamma} \bar{n}_{\gamma} \mathcal{D}_{\hat{b}_{\gamma}^{\dagger}}(\hat{\rho})
$$

with dissipator

$$
\mathcal{D}_{\hat{a}}(\hat{\rho}) \equiv \hat{a} \hat{\rho} \hat{a}^{\dagger}-\frac{1}{2}\left\{\hat{a}^{\dagger} \hat{a}, \hat{\rho}\right\}
$$

Here, the sum runs over all discrete phonon modes $\gamma,[\cdot, \cdot]$ indicates the commutator, and $\{\cdot, \cdot\}$ the anticommutator. This model captures the essential features of the discrete phonon modes: The steady state of the phonon modes in the absence of atom-phonon interaction is a thermal state $\hat{\sigma}_{\text {th }}$ with thermal phonon occupation $\bar{n}_{\gamma}$ for each mode [71]. Furthermore, if a phonon mode initially has occupation $n_{\gamma}^{0}$, it decays with rate $\kappa_{\gamma}$ back to the thermal phonon occupation $n_{\gamma}(t)=\bar{n}_{\gamma}+\left(n_{\gamma}^{0}-\bar{n}_{\gamma}\right) e^{-\kappa_{\gamma} t}$. We are interested in the effective dynamics of the atom density operator $\hat{\mu}$ under the assumption that the phonons remain in a thermal state. The total density operator is then $\hat{\rho}(t) \simeq \hat{\mu}(t) \otimes \hat{\sigma}_{\text {th }}$. Adiabatic elimination of the phonon d.o.f. in the limit of weak coupling compared to the phonon decay rates and atom and phonon frequencies $\kappa_{\gamma}, \omega_{\gamma}, \omega_{i} \gg g_{\gamma i}$ yields the master equation

$$
\frac{d}{d t} \hat{\mu}(t)=\frac{1}{i \hbar}\left[\hat{H}_{\mathrm{at}}, \hat{\mu}\right]+\sum_{i} \Gamma_{i}^{-} \mathcal{D}_{\hat{a}_{i}}(\hat{\mu})+\sum_{i} \Gamma_{i}^{+} \mathcal{D}_{\hat{a}_{i}^{\dagger}}(\hat{\mu})
$$

for the motion of the atom [73,74]. Here, $\hat{H}_{\text {at }}$ contains a small Lamb shift of the trap frequencies that is not relevant to our discussion. The phonon-induced decay $(-)$ and heating $(+)$ rates are

$$
\begin{aligned}
\Gamma_{i}^{ \pm} & \equiv 2 \sum_{\gamma}\left|g_{\gamma i}\right|^{2}\left[\bar{n}_{\gamma} G_{\gamma i}^{\mp}+\left(\bar{n}_{\gamma}+1\right) G_{\gamma i}^{ \pm}\right], \\
G_{\gamma i}^{ \pm} & \equiv \frac{2 \kappa_{\gamma}}{\kappa_{\gamma}^{2}+4\left(\omega_{i} \pm \omega_{\gamma}\right)^{2}} .
\end{aligned}
$$

If the atom is initially in the motional ground state and there is no cross-coupling between the motional directions $\left(g_{i j}=0\right)$, the population of its motion in direction $i$ evolves as $n_{i}(t)=n_{i}^{\infty}\left(1-e^{-\Gamma t}\right)$, with $\Gamma \equiv \Gamma_{i}^{-}-\Gamma_{i}^{+}$ and $n_{i}^{\infty} \equiv \Gamma_{i}^{+} / \Gamma$. At times $t \ll \Gamma$, the population grows 
linearly, with ground-state heating rate $\Gamma_{i}^{d}=\Gamma_{i}^{+}$. The total phonon-induced heating rate $\Gamma_{i}^{\text {th }}$ of the atomic motion along direction $i$ is then obtained according to Eq. (10) by summing $\Gamma_{i}^{d}$ with the contribution $\Gamma_{i}^{c}$ of the continuous modes in Eq. (D34).

One approach to reducing the atom heating rate is to optimize the nanofiber such that flexural modes are also reflected at the ends; see Sec. III. The flexural eigenmodes then become standing waves Eq. (35) with frequency spectrum $\omega_{m}, m \in \mathbb{N}$ given in Eq. (16), and decay rates $\kappa_{m}$. If the spacing between resonator frequencies is sufficiently large, the trap frequencies $\omega_{i}$ can be detuned from resonance with the flexural modes $\left|\omega_{i}-\omega_{m}\right| \gg \kappa_{m}$. The spacing of phonon frequencies close to the trap frequency is approximately $2 \sqrt{\omega_{i} \omega_{1}}+\omega_{1}$, where $\omega_{1}$ is the fundamental frequency of the resonator defined in Eq. (16); the shorter the resonator and the larger the fiber radius, the easier it is to detune the trap from resonance. Provided the coupling rates $g_{m i}$ between phonon mode $m$ and atomic motion in direction $i \in\{r, \varphi\}$ are smaller than phonon decay rates $\kappa_{m}$ and atom and phonon frequencies $g_{m i} \ll \kappa_{m}, \omega_{m}, \omega_{i}$, the effective dynamics of the atom is described by a master equation of the form Eq. (D39). The heating rate in the radial and azimuthal direction due to the flexural resonator modes of an atom at position $z_{0}$ is then

$$
\left.\Gamma_{i}^{\text {th }} \simeq 4 \sum_{m \in \mathbb{N}}\left|g_{m i}\left(z_{0}\right)\right|^{2}\left[\bar{n}_{m} G_{m i}^{-}+\left(\bar{n}_{m}+1\right) G_{m i}^{+}\right)\right],
$$

with a position-dependent displacement coupling constant

$$
g_{m i}(z)=-\frac{\omega_{i}}{2 R} \sqrt{\frac{M}{\pi L \rho} \frac{\omega_{i}}{\omega_{m}}} \sin \left(p_{m} z\right)
$$

and $G_{m i}^{ \pm}$as defined in Eq. (D40). The atom heating rate due to flexural resonances Eq. (D41) can be explicitly evaluated in different limiting cases, yielding the heating rates Eqs. (17)-(19) that we discuss in Sec. III.

\section{APPENDIX E: CASE STUDY PARAMETERS}

In nanofiber-based two-color atom traps, different combinations of linearly and circularly polarized trapping light fields are commonly used, both as propagating or standing waves [77]. In Appendix E1, we summarize the corresponding shapes of the electric field required for the atom heating case study in Sec. III. In Appendix E 2, we provide a listing of the parameters used in the case study based on Ref. [26].

\section{Monochromatic guided fields}

For each frequency $\omega$ on the $\mathrm{HE}_{|m| n}$ and $\mathrm{EH}_{|m| n}$ bands, there are four degenerate eigenmodes propagating in the positive $\left(\sigma_{z}=1\right)$ or negative $\left(\sigma_{z}=-1\right)$ direction along the $z$ axis and rotating with positive $\left(\sigma_{\varphi}=1\right)$ or negative $\left(\sigma_{\varphi}=-1\right)$ orientation around the fiber axis. The corresponding propagation constant is $k_{\sigma}=\sigma_{z} k$ with $k>0$, and the azimuthal order $m_{\sigma}=\sigma_{z} \sigma_{\varphi} m$ with $m>0$. The multiindex $\sigma=\left(\sigma_{\varphi}, \sigma_{z}\right)$ is used to distinguish the propagation and polarization state. Superposition of these four modes yields a monochromatic electromagnetic field

$$
\begin{aligned}
& \boldsymbol{E}(\boldsymbol{r}, t)=\boldsymbol{E}_{0}(\boldsymbol{r}) e^{-i \omega t}+\text { c.c. } \\
& \boldsymbol{B}(\boldsymbol{r}, t)=\boldsymbol{B}_{0}(\boldsymbol{r}) e^{-i \omega t}+\text { c.c. },
\end{aligned}
$$

with complex field profiles

$$
\begin{aligned}
& \boldsymbol{E}_{0}(\boldsymbol{r})=\sum_{\sigma} \alpha_{\sigma} \boldsymbol{e}_{m_{\sigma}}\left(k_{\sigma} ; \boldsymbol{r}\right), \\
& \boldsymbol{B}_{0}(\boldsymbol{r})=\sum_{\sigma} \alpha_{\sigma} \boldsymbol{b}_{m_{\sigma}}\left(k_{\sigma} ; \boldsymbol{r}\right) .
\end{aligned}
$$

Here, $\alpha_{\sigma} \in \mathbb{C}$ are amplitudes, and $\boldsymbol{e}_{m_{\sigma}}\left(k_{\sigma} ; \boldsymbol{r}\right), \boldsymbol{b}_{m_{\sigma}}\left(k_{\sigma} ; \boldsymbol{r}\right)$ are modal fields Eq. (A9) with radial partial waves listed in Table VI. We drop all irrelevant mode indices, keeping $m_{\sigma}$ and $k_{\sigma}$. The overall magnitude of the amplitudes is related to the power transmitted along the fiber axis $\boldsymbol{e}_{z}$,

$$
P=\int_{0}^{2 \pi} \int_{0}^{\infty} I(r, \varphi) d r d \varphi .
$$

Here, $I=\langle\boldsymbol{S}\rangle_{t} \cdot \boldsymbol{e}_{z}$ is light intensity in direction $\boldsymbol{e}_{z}$, and $\langle\boldsymbol{S}\rangle_{t}=2 \operatorname{Re}\left[\boldsymbol{E}_{0}(\boldsymbol{r}) \times \boldsymbol{B}_{0}^{*}(\boldsymbol{r})\right] / \mu_{0}$ is the Poynting vector averaged over an oscillation period. The star indicates the complex conjugate, and $\mu_{0}$ is the vacuum permeability.

A light field rotating with orientation $\sigma_{\varphi}$ around the fiber axis and propagating in direction $\sigma_{z} \boldsymbol{e}_{z}$ is realized by the amplitudes

$$
\alpha_{\sigma^{\prime}}=\left(\sigma_{\varphi} \sigma_{z}\right)^{m} 2 \pi \alpha e^{i \theta} \delta_{\sigma_{\varphi} \sigma_{\varphi}^{\prime}} \delta_{\sigma_{z} \sigma_{z}^{\prime}},
$$

where $\alpha \in \mathbb{R}$, and $\theta \in R$ is the overall phase of the wave. We include a factor of $2 \pi$ and the sign $\left(\sigma_{\varphi} \sigma_{z}\right)^{m}$ for later convenience. The field profile and resulting electric field are given by case (1) in Table XVII. The power transmitted along the fiber axis can be expressed as

$P=-\sigma_{z} \frac{4 \pi \alpha^{2}}{\mu_{0}} \int_{0}^{\infty} r\left[\mathcal{E}_{m}^{r}(k ; r) \mathcal{B}_{m}^{\varphi}(k ; r)+\mathcal{E}_{m}^{\varphi}(k ; r) \mathcal{B}_{m}^{r}(k ; r)\right] d r$

using the symmetries Eq. (A14).

A nonrotating light field propagating in direction $\sigma_{z}$ corresponds to the choice of amplitudes

$$
\alpha_{\sigma^{\prime}}=\left(\sigma_{\varphi}^{\prime} \sigma_{z}\right)^{m} 2 \pi \alpha e^{i\left(\sigma_{\varphi}^{\prime} \sigma_{z} \theta_{\varphi}+\theta\right)} \delta_{\sigma_{z} \sigma_{z}^{\prime}}
$$

and the resulting electric field is given by case (2) in Table XVII. The phase $\theta_{\varphi} \in \mathbb{R}$ determines the orientation 
TABLE XVII. Electric fields of monochromatic waves of frequency $\omega$ on the $\mathrm{HE}_{|m| n}$ and $\mathrm{EH}_{|m| n}$ bands. The sign $\sigma_{z}$ indicates the propagation direction along the fiber axis, the sign $\sigma_{\varphi}$ the rotation direction around the axis. The propagation constant is $k_{\sigma}=\sigma_{z} k$ with $k>0$, the azimuthal order $m_{\sigma}=\sigma_{\varphi} \sigma_{z} m$ with $m>0$, and the amplitude $\alpha \in \mathbb{R}$ is determined by the transmitted power. The quantities $\theta$, $\theta_{\varphi}, \theta_{z} \in \mathbb{R}$ are phases explained in the text. The radial partial waves $\mathcal{E}_{m}^{i}(k ; r)$ are listed in Table VI.

\begin{tabular}{lll}
\hline \hline Case & Field profile & Field \\
\hline
\end{tabular}

(1) Circular polarized running wave

$$
\begin{aligned}
& E_{0}^{r}=\sigma_{z} \alpha \mathcal{E}_{m}^{r}(k ; r) e^{i\left(m_{\sigma} \varphi+k_{\sigma} z+\theta\right)} \\
& E^{\varphi}=\sigma_{\varphi} \alpha \mathcal{E}_{m}^{\varphi}(k ; r) e^{i\left(m_{\sigma} \varphi+k_{\sigma} z+\theta\right)} \\
& E^{z}=\alpha \mathcal{E}_{m}^{z}(k ; r) e^{i\left(m_{\sigma} \varphi+k_{\sigma} z+\theta\right)}
\end{aligned}
$$

(2) Nonrotating running wave

$$
\begin{aligned}
& E_{0}^{r}=\sigma_{z} 2 \alpha \mathcal{E}_{m}^{r}(k ; r) \cos \left(m \varphi+\theta_{\varphi}\right) e^{i\left(k_{\sigma} z+\theta\right)} \\
& E_{0}^{\varphi}=\sigma_{z} 2 i \alpha \mathcal{E}_{m}^{\varphi}(k ; r) \sin \left(m \varphi+\theta_{\varphi}\right) e^{i\left(k_{\sigma} z+\theta\right)} \\
& E_{0}^{z}=2 \alpha \mathcal{E}_{m}^{z}(k ; r) \cos \left(m \varphi+\theta_{\varphi}\right) e^{i\left(k_{\sigma} z+\theta\right)}
\end{aligned}
$$

(3) Nonrotating standing wave

$$
\begin{aligned}
& E_{0}^{r}=4 i \alpha \mathcal{E}_{m}^{r}(k ; r) \cos \left(m \varphi+\theta_{\varphi}\right) \sin \left(k z+\theta_{z}\right) e^{i \theta} \\
& E_{0}^{\varphi}=-4 \alpha \mathcal{E}_{m}^{\varphi}(k ; r) \sin \left(m \varphi+\theta_{\varphi}\right) \sin \left(k z+\theta_{z}\right) e^{i \theta} \\
& E_{0}^{z}=4 \alpha \mathcal{E}_{m}^{z}(k ; r) \cos \left(m \varphi+\theta_{\varphi}\right) \cos \left(k z+\theta_{z}\right) e^{i \theta}
\end{aligned}
$$

$$
\begin{aligned}
& E^{r}=-\sigma_{z} 2 \alpha \operatorname{Im}\left[\mathcal{E}_{m}^{r}(k ; r)\right] \sin \left(m_{\sigma} \varphi+k_{\sigma} z-\omega t+\theta\right) \\
& E^{\varphi}=\sigma_{\varphi} 2 \alpha \mathcal{E}_{m}^{\varphi}(k ; r) \cos \left(m_{\sigma} \varphi+k_{\sigma} z-\omega t+\theta\right) \\
& E^{z}=2 \alpha \mathcal{E}_{m}^{z}(k ; r) \cos \left(m_{\sigma} \varphi+k_{\sigma} z-\omega t+\theta\right)
\end{aligned}
$$

$$
\begin{aligned}
& E^{r}=-\sigma_{z} 4 \alpha \operatorname{Im}\left[\mathcal{E}_{m}^{r}(k ; r)\right] \cos \left(m \varphi+\theta_{\varphi}\right) \sin \left(k_{\sigma} z-\omega t+\theta\right) \\
& E^{\varphi}=-\sigma_{z} 4 \alpha \mathcal{E}_{m}^{\varphi}(k ; r) \sin \left(m \varphi+\theta_{\varphi}\right) \sin \left(k_{\sigma} z-\omega t+\theta\right) \\
& E^{z}=4 \alpha \mathcal{E}_{m}^{z}(k ; r) \cos \left(m \varphi+\theta_{\varphi}\right) \cos \left(k_{\sigma} z-\omega t+\theta\right)
\end{aligned}
$$

$$
\begin{aligned}
& E^{r}=-8 \alpha \operatorname{Im}\left[\mathcal{E}_{m}^{r}(k ; r)\right] \cos \left(m \varphi+\theta_{\varphi}\right) \sin \left(k z+\theta_{z}\right) \cos (\omega t+\theta) \\
& E^{\varphi}=-8 \alpha \mathcal{E}_{m}^{\varphi}(k ; r) \sin \left(m \varphi+\theta_{\varphi}\right) \sin \left(k z+\theta_{z}\right) \cos (\omega t+\theta) \\
& E^{z}=8 \alpha \mathcal{E}_{m}^{z}(k ; r) \cos \left(m \varphi+\theta_{\varphi}\right) \cos \left(k z+\theta_{z}\right) \cos (\omega t+\theta)
\end{aligned}
$$

of the wave in the $(x, y)$ plane. For azimuthal order $|m|=1$ in particular, the electric field is mainly oriented along an axis in the $(x, y)$ plane that encloses the angle $\theta_{\varphi}$ with the $x$ axis. These waves are therefore called quasilinear polarized. The transmitted power is $2 P$ as given in Eq. (E5).

Two counterpropagating quasilinear waves create a

\begin{tabular}{|c|c|c|c|c|c|c|}
\hline Parameter & Description & Source & Parameter & Description & Source & \\
\hline \multicolumn{7}{|l|}{ Mechanical } \\
\hline$R=250 \mathrm{~nm}$ & Fiber radius & [26] & $\mu=31.2 \mathrm{GPa}$ & Second Lamé coefficient & (C2) & $\star$ \\
\hline$E=72.6 \mathrm{GPa}$ & Young's modulus & {$[115]$} & $c_{l}=5.94 \times 10^{3} \mathrm{~m} / \mathrm{s}$ & Longitudinal sound speed & $(\mathrm{C} 15)$ & $\star$ \\
\hline$\nu=0.164$ & Poisson's ratio & {$[115]$} & $c_{t}=3.76 \times 10^{3} \mathrm{~m} / \mathrm{s}$ & Transverse sound speed & $(\mathrm{C} 15)$ & $\star$ \\
\hline$\rho=2.20 \mathrm{~g} / \mathrm{cm}^{3}$ & Mass density & [115] & $c_{h}=5.74 \times 10^{3} \mathrm{~m} / \mathrm{s}$ & Effective sound speed & (C31) & $\star$ \\
\hline$\lambda=15.2 \mathrm{GPa}$ & First Lamé coefficient & $(\mathrm{C} 2)$ & $\star$ & & & \\
\hline$\omega_{T} / 2 \pi=258 \mathrm{kHz}$ & Fundamental frequency & & $\kappa / 2 \pi=48.0 \mathrm{~Hz}$ & Decay rate & & \\
\hline$Q=5380$ & Quality factor & & $\star L=7.29 \mathrm{~mm}$ & Effective nanofiber length & (E8) & $\star$ \\
\hline \multicolumn{7}{|l|}{ Optical } \\
\hline$\epsilon=2.1$ & Relative permittivity & {$[4,115]$} & & & & \\
\hline$\lambda_{\mathrm{r}}=1064 \mathrm{~nm}$ & Free-space wavelength & {$[26]$} & $\lambda_{b}=783 \mathrm{~nm}$ & Free-space wavelength & [26] & \\
\hline$\omega_{r} / 2 \pi=282 \mathrm{THz}$ & Angular frequency & & $\star \omega_{b} / 2 \pi=383 \mathrm{THz}$ & Angular frequency & & $\star$ \\
\hline
\end{tabular}
standing wave corresponding to the amplitudes

$$
\alpha_{\sigma}=\left(\sigma_{\varphi} \sigma_{z}\right)^{m} 2 \pi \alpha e^{i\left(\sigma_{\varphi} \sigma_{z} \theta_{\varphi}+\sigma_{z} \theta_{z}+\theta\right)} .
$$

The phase $\theta_{z} \in \mathbb{R}$ determines the position of nodes of the standing wave along the fiber axis. The electric field is given by case (3) in Table XVII. The power transmitted along the fiber axis vanishes, but each counterpropagating wave has again power $2 P$ as given in Eq. (E5).

\section{Physical parameters}

In Table XVIII we list the parameters used in the case study Sec. III based on the setup described in Ref. [26]. Citations are given in square brackets and references to equations used to calculate dependent parameters in parentheses. A star indicates that a parameter depends on previously chosen parameters.

TABLE XVIII. Parameters for the case study in Sec. III. A star $(\star)$ indicates that a parameter depends on previously chosen parameters. 
TABLE XVIII. (Continued)

\begin{tabular}{|c|c|c|c|c|c|c|}
\hline Parameter & Description & Source & Parameter & Description & Source & \\
\hline$\left|k_{r}\right|=6.31 \mu \mathrm{m}^{-1}$ & Propagation constant & (A12) & $\star\left|k_{b}\right|=9.41 \mu \mathrm{m}^{-1}$ & Propagation constant & (A12) & $\star$ \\
\hline$\left|P_{r}\right|=1.25 \mathrm{~mW}$ & Power & {$[26]$} & $\left|P_{b}\right|=17.8 \mathrm{~mW}$ & Power & {$[26]$} & \\
\hline$\alpha_{r}=1.41 \mathrm{pAs} / \mathrm{m}$ & Amplitude & (E3) & $\star \alpha_{b}=5.70 \mathrm{pAs} / \mathrm{m}$ & Amplitude & (E3) & $\star$ \\
\hline$\theta_{\varphi}^{r}=0$ & $\varphi$ phase shift & & $\theta_{\varphi}^{b}=\pi / 2$ & $\varphi$ phase shift & & $\star$ \\
\hline$\theta_{z}^{r}=\pi / 2-k_{r} L / 2$ & $z$ phase shift & & & & & \\
\hline$P_{1}=0.100$ & Photoelasticity & {$[112,116]$} & $P_{2}=0.285$ & Photoelasticity & {$[112,116]$} & \\
\hline \multicolumn{7}{|l|}{ Atomic } \\
\hline$M=2.21 \times 10^{-25} \mathrm{~kg}$ & Mass & [117] & $\star$ & & & \\
\hline$F=4$ & HFS state & {$[26]$} & $M_{F}=-4$ & Zeeman substate & [26] & \\
\hline$\tilde{\alpha}_{s}^{r}=1164$ a.u. & FS scalar polarization at $\omega_{r}$ & [94] & $\star \tilde{\alpha}_{s}^{b}=-1761.6$ a.u. & FS scalar polarization at $\omega_{b}$ & [94] & $\star$ \\
\hline$\tilde{\alpha}_{v}^{r}=-198.64$ a.u. & FS vector polarization at $\omega_{r}$ & [94] & $\star \tilde{\alpha}_{v}^{b}=-479.96$ a.u. & FS vector polarization at $\omega_{b}$ & [94] & $\star$ \\
\hline$\tilde{\alpha}_{t}^{r}=0$ & FS tensor polarization at $\omega_{r}$ & {$[94]$} & $\star \tilde{\alpha}_{t}^{b}=0$ & FS tensor polarization at $\omega_{b}$ & [94] & $\star$ \\
\hline$C / h=1.178 \mathrm{THz} \mathrm{nm}{ }^{3}$ & Strength dispersion force & {$[101]$} & & & & \\
\hline \multicolumn{7}{|l|}{ Trap } \\
\hline$r_{0}=553 \mathrm{~nm}$ & Trap position & & $\star z_{0}=L / 2$ & & & $\star$ \\
\hline$\varphi_{0}=-0.0190 \mathrm{rad}$ & & & $\star V_{0} / h=-3.21 \mathrm{MHz}$ & Trap depth & & $\star$ \\
\hline$\omega_{r} / 2 \pi=123 \mathrm{kHz}$ & Trap frequency & (B12) & $\star \Delta r=17.6 \mathrm{~nm}$ & Zero-point motion & (B13) & $\star$ \\
\hline$\omega_{\varphi} / 2 \pi=71.8 \mathrm{kHz}$ & & (B12) & $\star r_{0} \Delta \varphi=23.0 \mathrm{~nm}$ & & (B13) & $\star$ \\
\hline$\omega_{z} / 2 \pi=193 \mathrm{kHz}$ & & (B12) & $\star \Delta z=14.0 \mathrm{~nm}$ & & (B13) & $\star$ \\
\hline
\end{tabular}

The mechanical properties of the silica fiber are determined by the choice of material. The frequencies of the discrete torsional modes confined to the nanofiber are determined experimentally, as we describe in Sec. III. The (effective) length $L$ of the nanofiber for torsional modes is inferred from the measured frequency $\omega_{T}$ of the fundamental torsional mode using Eq. (28):

$$
L=c_{t} \pi / \omega_{T}
$$

The optical properties of the fiber are determined by permittivity and radius. The power of the red-detuned field quoted below corresponds to each beam separately. The coordinate system is chosen such that the red-detuned laser beam is polarized along the $x$ axis. This is reflected in the choice of azimuthal phase shifts $\theta_{\varphi}$. Moreover, the axial phase shift $\theta_{z}$ is chosen such that there is a trapping site at $z=L / 2$ in the middle of the fiber. The magnetic offset field $\boldsymbol{B}_{\text {ext }}$ is oriented perpendicular to the fiber axis, along

$$
z_{B}=\cos (\phi) \boldsymbol{e}_{x}+\sin (\phi) \boldsymbol{e}_{y}
$$

with $\phi=66^{\circ}$. The potential experienced by the atom depends on its mass and the polarizability of its internal hyperfine-structure state; see Appendix B. The values in Table XVIII correspond to a ${ }_{55}^{133} \mathrm{Cs}$ atom in the ground state $6^{2} S_{1 / 2}$ interacting with the red-detuned $(r)$ and bluedetuned $(b)$ light field. The atomic unit of polarizability is 1 a.u. $=\left(4 \pi \epsilon_{0}\right)^{4} \hbar^{6} /\left(m_{\mathrm{el}}^{3} e^{6}\right)=1.65 \times 10^{-41} \quad \mathrm{~A}^{2} \mathrm{~s}^{4} / \mathrm{kg}$. The resulting atom trapping site $\boldsymbol{r}_{0}$ indicated in Fig. 4 is slightly shifted away from the $x$ axis by vector light shifts due to the orientation of the magnetic offset field.

[1] J. D. Thompson, T. G. Tiecke, N. P. de Leon, J. Feist, A. V. Akimov, M. Gullans, A. S. Zibrov, V. Vuletić, and M. D. Lukin, Coupling a Single Trapped Atom to a Nanoscale Optical Cavity, Science 340, 1202 (2013).

[2] A. Goban, C.-L. Hung, J. D. Hood, S.-P. Yu, J. A. Muniz, O. Painter, and H. J. Kimble, Superradiance for Atoms Trapped along a Photonic Crystal Waveguide, Phys. Rev. Lett. 115, 063601 (2015).

[3] L. Magrini, R. A. Norte, R. Riedinger, I. Marinković, D. Grass, U. Delić, S. Gröblacher, S. Hong, and M. Aspelmeyer, Near-Field Coupling of a Levitated Nanoparticle to a Photonic Crystal Cavity, Optica 5, 1597 (2018).

[4] E. Vetsch, D. Reitz, G. Sagué, R. Schmidt, S. T. Dawkins, and A. Rauschenbeutel, Optical Interface Created by Laser-Cooled Atoms Trapped in the Evanescent Field Surrounding an Optical Nanofiber, Phys. Rev. Lett. 104, 203603 (2010).

[5] A. Goban, K. S. Choi, D. J. Alton, D. Ding, C. Lacroûte, M. Pototschnig, T. Thiele, N. P. Stern, and H. J. Kimble, Demonstration of a State-Insensitive, Compensated Nanofiber Trap, Phys. Rev. Lett. 109, 033603 (2012).

[6] J.-B. Béguin, E. M. Bookjans, S. L. Christensen, H. L. Sørensen, J. H. Müller, E. S. Polzik, and J. Appel, Generation 
and Detection of a Sub-Poissonian Atom Number Distribution in a One-Dimensional Optical Lattice, Phys. Rev. Lett. 113, 263603 (2014).

[7] S. Kato and T. Aoki, Strong Coupling between a Trapped Single Atom and an All-Fiber Cavity, Phys. Rev. Lett. 115, 093603 (2015).

[8] J. Lee, J. A. Grover, J. E. Hoffman, L. A. Orozco, and S. L. Rolston, Inhomogeneous Broadening of Optical Transitions of $87 \mathrm{Rb}$ Atoms in an Optical Nanofiber Trap, J. Phys. B 48, 165004 (2015).

[9] N. V. Corzo, B. Gouraud, A. Chandra, A. Goban, A. S. Sheremet, D. V. Kupriyanov, and J. Laurat, Large Bragg Reflection from One-Dimensional Chains of Trapped Atoms Near a Nanoscale Waveguide, Phys. Rev. Lett. 117, 133603 (2016).

[10] M. Gierling, P. Schneeweiss, G. Visanescu, P. Federsel, M. Häffner, D. P. Kern, T. E. Judd, A. Günther, and J. Fortágh, Cold-Atom Scanning Probe Microscopy, Nat. Nanotechnol. 6, 446 (2011).

[11] P. Schneeweiss, M. Gierling, G. Visanescu, D. P. Kern, T. E. Judd, A. Günther, and J. Fortágh, Dispersion Forces between Ultracold Atoms and a Carbon Nanotube, Nat. Nanotechnol. 7, 515 (2012).

[12] R. Diehl, E. Hebestreit, R. Reimann, F. Tebbenjohanns, M. Frimmer, and L. Novotny, Optical Levitation and Feedback Cooling of a Nanoparticle at Subwavelength Distances from a Membrane, Phys. Rev. A 98, 013851 (2018).

[13] M. Hammes, D. Rychtarik, H.-C. Nägerl, and R. Grimm, Cold-Atom Gas at Very High Densities in an Optical Surface Microtrap, Phys. Rev. A 66, 051401(R) (2002).

[14] H. Bender, C. Stehle, C. Zimmermann, S. Slama, J. Fiedler, S. Scheel, S. Y. Buhmann, and V. N. Marachevsky, Probing Atom-Surface Interactions by Diffraction of BoseEinstein Condensates, Phys. Rev. X 4, 011029 (2014).

[15] A. A. Geraci, S. J. Smullin, D. M. Weld, J. Chiaverini, and A. Kapitulnik, Improved Constraints on Non-Newtonian Forces at 10 Microns, Phys. Rev. D 78, 022002 (2008).

[16] A. A. Geraci, S. B. Papp, and J. Kitching, Short-Range Force Detection Using Optically Cooled Levitated Microspheres, Phys. Rev. Lett. 105, 101101 (2010).

[17] N. Arkani-Hamed, S. Dimopoulos, and G. Dvali, The Hierarchy Problem and New Dimensions at a Millimeter, Phys. Lett. B 429, 263 (1998).

[18] Casimir Physics, edited by D. Dalvit, P. Milonni, D. Roberts, and F. da Rosa (Springer, Berlin, 2011).

[19] G. L. Klimchitskaya, U. Mohideen, and V. M. Mostepanenko, The Casimir Force between Real Materials: Experiment and Theory, Rev. Mod. Phys. 81, 1827 (2009).

[20] J.-B. Béguin, J. H. Müller, J. Appel, and E. S. Polzik, Observation of Quantum Spin Noise in a $1 D$ Light-Atoms Quantum Interface, Phys. Rev. X 8, 031010 (2018).

[21] C. Sayrin, C. Clausen, B. Albrecht, P. Schneeweiss, and A. Rauschenbeutel, Storage of Fiber-Guided Light in a Nanofiber-Trapped Ensemble of Cold Atoms, Optica 2, 353 (2015).

[22] B. Gouraud, D. Maxein, A. Nicolas, O. Morin, and J. Laurat, Demonstration of a Memory for Tightly Guided Light in an Optical Nanofiber, Phys. Rev. Lett. 114, 180503 (2015).
[23] N. V. Corzo, J. Raskop, A. Chandra, A. S. Sheremet, B. Gouraud, and J. Laurat, Waveguide-Coupled Single Collective Excitation of Atomic Arrays, Nature (London) 566, 359 (2019).

[24] Y. Meng, A. Dareau, P. Schneeweiss, and A. Rauschenbeutel, Near-Ground-State Cooling of Atoms Optically Trapped 300 nm Away from a Hot Surface, Phys. Rev. X 8, 031054 (2018).

[25] D. Reitz, C. Sayrin, R. Mitsch, P. Schneeweiss, and A. Rauschenbeutel, Coherence Properties of NanofiberTrapped Cesium Atoms, Phys. Rev. Lett. 110, 243603 (2013).

[26] B. Albrecht, Y. Meng, C. Clausen, A. Dareau, P. Schneeweiss, and A. Rauschenbeutel, Fictitious Magnetic-Field Gradients in Optical Microtraps as an Experimental Tool for Interrogating and Manipulating Cold Atoms, Phys. Rev. A 94, 061401(R) (2016).

[27] P. Schneeweiss, A. Dareau, and C. Sayrin, Cold-AtomBased Implementation of the Quantum Rabi Model, Phys. Rev. A 98, 021801(R) (2018).

[28] A. Dareau, Y. Meng, P. Schneeweiss, and A. Rauschenbeutel, Observation of Ultrastrong Spin-Motion Coupling for Cold Atoms in Optical Microtraps, Phys. Rev. Lett. 121, 253603 (2018).

[29] R. Engelbrecht, Nichtlineare Faseroptik: Grundlagen und Anwendungsbeispiele (Springer, New York, 2015).

[30] J.-C. Beugnot, S. Lebrun, G. Pauliat, H. Maillotte, V. Laude, and T. Sylvestre, Brillouin Light Scattering from Surface Acoustic Waves in a Subwavelength-Diameter Optical Fibre, Nat. Commun. 5, 5242 (2014).

[31] O. Florez, P. F. Jarschel, Y. a. V. Espinel, C. M. B. Cordeiro, T. P. Mayer Alegre, G. S. Wiederhecker, and P. Dainese, Brillouin Scattering Self-Cancellation, Nat. Commun. 7, 11759 (2016).

[32] C. Henkel, S. Pötting, and M. Wilkens, Loss and Heating of Particles in Small and Noisy Traps, Appl. Phys. B 69, 379 (1999).

[33] See Supplemental Material at http://link.aps.org/ supplemental/10.1103/PhysRevX.9.041034 for estimates of the contribution of other mechanisms to the heating of nanofiber-trapped cold atoms.

[34] C. Wuttke, G. D. Cole, and A. Rauschenbeutel, Optically Active Mechanical Modes of Tapered Optical Fibers, Phys. Rev. A 88, 061801(R) (2013).

[35] D. Rychtarik, B. Engeser, H.-C. Nägerl, and R. Grimm, Two-Dimensional Bose-Einstein Condensate in an Optical Surface Trap, Phys. Rev. Lett. 92, 173003 (2004).

[36] C. Henkel and M. Wilkens, Heating of Trapped Atoms near Thermal Surfaces, Europhys. Lett. 47, 414 (1999).

[37] D. E. Chang, C. A. Regal, S. B. Papp, D. J. Wilson, J. Ye, O. Painter, H. J. Kimble, and P. Zoller, Cavity Opto-Mechanics Using an Optically Levitated Nanosphere, Proc. Natl. Acad. Sci. U.S.A. 107, 1005 (2010).

[38] O. Romero-Isart, M. L. Juan, R. Quidant, and J. I. Cirac, Toward Quantum Superposition of Living Organisms, New J. Phys. 12, 033015 (2010).

[39] T. Li, S. Kheifets, and M. G. Raizen, Millikelvin Cooling of an Optically Trapped Microsphere in Vacuum, Nat. Phys. 7, 527 (2011).

[40] J. Gieseler, B. Deutsch, R. Quidant, and L. Novotny, Subkelvin Parametric Feedback Cooling of 
a Laser-Trapped Nanoparticle, Phys. Rev. Lett. 109, 103603 (2012).

[41] N. Kiesel, F. Blaser, U. Delić, D. Grass, R. Kaltenbaek, and M. Aspelmeyer, Cavity Cooling of an Optically Levitated Submicron Particle, Proc. Natl. Acad. Sci. U.S.A. 110, 14180 (2013).

[42] P. Z. G. Fonseca, E. B. Aranas, J. Millen, T. S. Monteiro, and P. F. Barker, Nonlinear Dynamics and Strong Cavity Cooling of Levitated Nanoparticles, Phys. Rev. Lett. 117, 173602 (2016).

[43] V. Jain, J. Gieseler, C. Moritz, C. Dellago, R. Quidant, and L. Novotny, Direct Measurement of Photon Recoil from a Levitated Nanoparticle, Phys. Rev. Lett. 116, 243601 (2016).

[44] R. Grimm, M. Weidemüller, and Y. B. Ovchinnikov, Optical Dipole Traps for Neutral Atoms, Adv. At. Mol. Opt. Phys. 42, 95 (2000).

[45] D. E. Chang, J. S. Douglas, A. González-Tudela, C.-L. Hung, and H. J. Kimble, Colloquium: Quantum Matter Built from Nanoscopic Lattices of Atoms and Photons, Rev. Mod. Phys. 90, 031002 (2018).

[46] H. Mabuchi and H. J. Kimble, Atom Galleries for Whispering Atoms: Binding Atoms in Stable Orbits around an Optical Resonator, Opt. Lett. 19, 749 (1994).

[47] J. P. Dowling and J. Gea-Banacloche, Evanescent LightWave Atom Mirrors, Resonators, Waveguides, and Traps, Adv. At. Mol. Opt. Phys. 37, 1 (1996).

[48] D. W. Vernooy and H. J. Kimble, Quantum Structure and Dynamics for Atom Galleries, Phys. Rev. A 55, 1239 (1997).

[49] F. Le Kien, V. I. Balykin, and K. Hakuta, Atom Trap and Waveguide Using a Two-Color Evanescent Light Field around a Subwavelength-Diameter Optical Fiber, Phys. Rev. A 70, 063403 (2004).

[50] C. A. Christensen, S. Will, M. Saba, G.-B. Jo, Y.-I. Shin, W. Ketterle, and D. Pritchard, Trapping of Ultracold Atoms in a Hollow-Core Photonic Crystal Fiber, Phys. Rev. A 78, 033429 (2008).

[51] C.-L. Hung, S. M. Meenehan, D. E. Chang, O. Painter, and H. J. Kimble, Trapped Atoms in One-Dimensional Photonic Crystals, New J. Phys. 15, 083026 (2013).

[52] A. Goban, C.-L. Hung, S.-P. Yu, J. D. Hood, J. A. Muniz, J. H. Lee, M. J. Martin, A. C. McClung, K. S. Choi, D. E. Chang, O. Painter, and H. J. Kimble, Atom-Light Interactions in Photonic Crystals, Nat. Commun. 5, 3808 (2014).

[53] Y. B. Ovchinnikov, S. V. Shul'ga, and V. I. Balykin, An Atomic Trap Based on Evanescent Light Waves, J. Phys. B 24, 3173 (1991).

[54] F. Le Kien and K. Hakuta, Microtraps for Atoms Outside a Fiber Illuminated Perpendicular to Its Axis: Numerical Results, Phys. Rev. A 80, 013415 (2009).

[55] C. Wuttke and A. Rauschenbeutel, Thermalization via Heat Radiation of an Individual Object Thinner than the Thermal Wavelength, Phys. Rev. Lett. 111, 024301 (2013).

[56] S. Y. Buhmann, Dispersion Forces I: Macroscopic Quantum Electrodynamics and Ground-State Casimir, Casimir-Polder and van der Waals Forces (Springer, Berlin, 2012).
[57] S. Fuchs, R. Bennett, R. V. Krems, and S. Y. Buhmann, Nonadditivity of Optical and Casimir-Polder Potentials, Phys. Rev. Lett. 121, 083603 (2018).

[58] J. D. Achenbach, Wave Propagation in Elastic Solids (North-Holland Publishing, Amsterdam, 1973).

[59] B. A. Auld, Acoustic Fields and Waves in Solids (John Wiley \& Sons, New York, 1973), Vol. 2.

[60] M. E. Gurtin, The Linear Theory of Elasticity, in Linear Theories of Elasticity and Thermoelasticity, Linear and Nonlinear Theories of Rods, Plates, and Shells, Mechanics of Solids, Vol. 2, edited by C. Truesdell (Springer, Berlin, 1984).

[61] H. Zoubi and K. Hammerer, Optomechanical Multimode Hamiltonian for Nanophotonic Waveguides, Phys. Rev. A 94, 053827 (2016).

[62] The Fréchet derivative $D F$ of a functional $F[\boldsymbol{x}]$ evaluated at $\boldsymbol{x}=\boldsymbol{a}$ and in direction $\boldsymbol{n}$ is defined as $[63,64]$

$$
D F_{\boldsymbol{a}}[\boldsymbol{n}] \equiv \lim _{h \rightarrow 0}\{F[\boldsymbol{a}+h \boldsymbol{n}]-F[\boldsymbol{a}]\} / h .
$$

The derivative is linear in $\boldsymbol{n}$, and can be used in a Taylor expansion [64]. In particular, it is suitable for the linearorder approximation $F[\boldsymbol{x}] \simeq F[\boldsymbol{a}]+D F_{\boldsymbol{a}}[\boldsymbol{x}]$. The partial Fréchet derivative $\delta_{x} G$ of a multivariate functional $G[\boldsymbol{x}, \boldsymbol{y}]$ with respect to $\boldsymbol{x}$ evaluated at $(\boldsymbol{x}, \boldsymbol{y})=(\boldsymbol{a}, \boldsymbol{b})$ and in direction $\boldsymbol{n}$ is defined as [63]

$$
\delta_{x} G_{(\boldsymbol{a}, \boldsymbol{b})}[\boldsymbol{n}] \equiv \lim _{h \rightarrow 0}\{G[\boldsymbol{a}+h \boldsymbol{n}, \boldsymbol{b}]-G[\boldsymbol{a}, \boldsymbol{b}]\} / h .
$$

Partial derivatives can be used to express the total derivative $D$ of a multivariate functional [63], for instance,

$$
D G_{(\boldsymbol{a}, \boldsymbol{b})}[\boldsymbol{n}, \boldsymbol{m}]=\delta_{x} G_{(\boldsymbol{a}, \boldsymbol{b})}[\boldsymbol{n}]+\delta_{y} G_{(\boldsymbol{a}, \boldsymbol{b})}[\boldsymbol{m}]
$$

in the case of a bivariate functional.

[63] S. Yamamuro, Differential Calculus in Topological Linear Spaces, Lecture Notes in Mathematics No. 374 (Springer, Berlin, 1974).

[64] D. Werner, Funktionalanalysis, 7th ed. (Springer, Heidelberg, 2011).

[65] The term $D V_{(\mathbf{0 , 0})}[\hat{\boldsymbol{u}}, \hat{\boldsymbol{S}}]\left(\boldsymbol{r}_{0}\right)$ at order zero in the expansion describes a light-induced change in the mechanical equilibrium configuration of the photonic structure. We may safely neglect this constant shift because it is small compared to the dimensions of a nanoscale structure and therefore weakly modifies only its photonic and phononic spectrum.

[66] S. G. Johnson, M. Ibanescu, M. A. Skorobogatiy, O. Weisberg, J. D. Joannopoulos, and Y. Fink, Perturbation Theory for Maxwell's Equations with Shifting Material Boundaries, Phys. Rev. E 65, 066611 (2002).

[67] F. Le Kien, S. D. Gupta, and K. Hakuta, Phonon-Mediated Decay of an Atom in a Surface-Induced Potential, Phys. Rev. A 75, 062904 (2007).

[68] D. F. Nelson and M. Lax, Theory of the Photoelastic Interaction, Phys. Rev. B 3, 2778 (1971).

[69] T. S. Narasimhamurty, Photoelastic and Electro-Optic Properties of Crystals (Springer Science \& Business Media, New York, 2012).

[70] C. Cohen-Tannoudji, J. Dupont-Roc, and G. Grynberg, Atom-Photon Interactions: Basic Processes and Applications (Wiley-VCH, Weinheim, 1998). 
[71] H.-P. Breuer and F. Petruccione, The Theory of Open Quantum Systems (Oxford University Press, New York, 2002).

[72] C. Gerry and P. Knight, Introductory Quantum Optics (Cambridge University Press, Cambridge, England, 2005).

[73] J. I. Cirac, R. Blatt, P. Zoller, and W. D. Phillips, Laser Cooling of Trapped Ions in a Standing Wave, Phys. Rev. A 46, 2668 (1992).

[74] I. Wilson-Rae, N. Nooshi, J. Dobrindt, T. J. Kippenberg, and W. Zwerger, Cavity-Assisted Backaction Cooling of Mechanical Resonators, New J. Phys. 10, 095007 (2008).

[75] J. M. Ward, A. Maimaiti, V. H. Le, and S. N. Chormaic, Optical Micro- and Nanofiber Pulling Rig, Rev. Sci. Instrum. 85, 111501 (2014).

[76] C. Østfeldt, J.-B. S. Béguin, F. T. Pedersen, E. S. Polzik, J. H. Müller, and J. Appel, Dipole Force Free Optical Control and Cooling of Nanofiber Trapped Atoms, Opt. Lett. 42, 4315 (2017).

[77] F. Le Kien, P. Schneeweiss, and A. Rauschenbeutel, StateDependent Potentials in a Nanofiber-Based Two-Color Trap for Cold Atoms, Phys. Rev. A 88, 033840 (2013).

[78] C. Wuttke, Thermal Excitations of Optical Nanofibers Measured with a Fiber-Integrated Fabry-Pérot Cavity, Ph.D. thesis, Johannes Gutenberg Unversität Mainz, 2013.

[79] R. Pennetta, S. Xie, and P. S. J. Russell, Tapered GlassFiber Microspike: high-Q Flexural Wave Resonator and Optically Driven Knudsen Pump, Phys. Rev. Lett. 117, 273901 (2016).

[80] J. Wiedersich, S. V. Adichtchev, and E. Rössler, Spectral Shape of Relaxations in Silica Glass, Phys. Rev. Lett. 84, 2718 (2000).

[81] S. D. Penn, A. Ageev, D. Busby, G. M. Harry, A. M. Gretarsson, K. Numata, and P. Willems, Frequency and Surface Dependence of the Mechanical Loss in Fused Silica, Phys. Lett. A 352, 3 (2006).

[82] E. F. Fenton, A. Khan, P. Solano, L. A. Orozco, and F. K. Fatemi, Spin-Optomechanical Coupling between Light and a Nanofiber Torsional Mode, Opt. Lett. 43, 1534 (2018).

[83] S. Spinner, Elastic Moduli of Glasses at Elevated Temperatures by a Dynamic Method, J. Am. Ceram. Soc. 39, 113 (1956).

[84] S. Ravets, J. E. Hoffman, P. R. Kordell, J. D. WongCampos, S. L. Rolston, and L. A. Orozco, Intermodal Energy Transfer in a Tapered Optical Fiber: Optimizing Transmission, J. Opt. Soc. Am. A 30, 2361 (2013).

[85] J. D. Jackson, Classical Electrodynamics, 3rd ed. (Wiley \& Sons, New York, 1999).

[86] J. D. Joannopoulos, S. G. Johnson, J. N. Winn, and R. D. Meade, Photonic Crystals: Molding the Flow of Light, 2nd ed. (Princeton University Press, Princeton, NJ, 2011).

[87] A. W. Snyder and J. Love, Optical Waveguide Theory (Springer, New York, 2012).

[88] D. Marcuse, Light Transmission Optics (Van Nostrand Reinhold, New York, 1982).

[89] C. C. Davis, Lasers and Electro-Optics: Fundamentals and Engineering (Cambridge University Press, Cambridge, England, 1996).

[90] R. J. Glauber and M. Lewenstein, Quantum Optics of Dielectric Media, Phys. Rev. A 43, 467 (1991).
[91] C. Cohen-Tannoudji, J. Dupont-Roc, and G. Grynberg, Photons and Atoms: Introduction to Quantum Electrodynamics (Wiley-VCH, Weinheim, 2004).

[92] This equation can be derived by starting from the Lagrange density $\mathcal{L}=\epsilon_{0}(\boldsymbol{\rho} \dot{\boldsymbol{A}})^{2} / 2-(\nabla \times \boldsymbol{A})^{2} / 2 \mu_{0}$ adapted from Ref. [90] for anisotropic media, passing to the Hamilton formulation, and expanding all fields in terms of the eigenmodes [91]. The tensor $\boldsymbol{\rho}$ here is the root of the permittivity $\rho^{2}=\boldsymbol{\epsilon}$, and the properties of the permittivity ensure that the root is unique, positive definite, and symmetric. The Hamilton functional is then of the form $H=\epsilon_{0}^{-1} \sum_{\eta}\left(\alpha_{\eta} \alpha_{\eta}^{*}+\alpha_{\eta}^{*} \alpha_{\eta}\right)$.

[93] A. W. Snyder, Continuous Mode Spectrum of a Circular Dielectric Rod, IEEE Trans. Microwave Theory Tech. 19, 720 (1971).

[94] F. Le Kien, P. Schneeweiss, and A. Rauschenbeutel, Dynamical Polarizability of Atoms in Arbitrary Light Fields: General Theory and Application to Cesium, Eur. Phys. J. D 67, 92 (2013).

[95] At atomic distances from the surface, strong repulsion due to exchange energy can arise. Together with attractive dispersion forces, this can lead to adsorption of the atom on the surface $[96,97]$.

[96] H. J. Kreuzer and Z. Gortel, Physisorption Kinetics (Springer, Berlin, 1986).

[97] A. Zangwill, Physics at Surfaces (Cambridge University Press, Cambridge, England, 1988).

[98] M. Schmeits and A. A Lucas, Physical Adsorption and Surface Plasmons, Surf. Sci. 64, 176 (1977).

[99] V. M. Nabutovskii, V. R. Belosludov, and A. M. Korotkikh, Interaction Potential between Small Neutral Particles and Spherical or Cylindrical Surfaces, J. Exp. Theor. Phys. 50, 352 (1979), http://www.jetp.ac.ru/cgi-bin/e/index/e/50/2/ p352?a=list.

[100] M. Boustimi, J. Baudon, P. Candori, and J. Robert, van der Waals Interaction between an Atom and a Metallic Nanowire, Phys. Rev. B 65, 155402 (2002).

[101] N. P. Stern, D. J. Alton, and H. J. Kimble, Simulations of Atomic Trajectories near a Dielectric Surface, New J. Phys. 13, 085004 (2011).

[102] A. D. McLachlan, van der Waals Forces between an Atom and a Surface, Mol. Phys. 7, 381 (1964).

[103] J. M. Wylie and J. E. Sipe, Quantum Electrodynamics near an Interface, Phys. Rev. A 30, 1185 (1984).

[104] K. Kustura, C. C. Rusconi, and O. Romero-Isart, Quadratic Quantum Hamiltonians: General Canonical Transformation to a Normal Form, Phys. Rev. A 99, 022130 (2019).

[105] The coupling between directions $i$ and $j \neq i$ can always be transformed away by selecting coordinates aligned with the symmetry axes of the potential in harmonic approximation.

[106] The strain energy can be rewritten by partial integration as $\int_{B} \sum_{i j} S^{i j} T^{i j} d \boldsymbol{r}=\int_{\partial B} \boldsymbol{u} \cdot(\boldsymbol{T} \boldsymbol{n}) d \partial B-\int_{B} \boldsymbol{u} \cdot \mathcal{D} \boldsymbol{u} d \boldsymbol{r}$, where $\partial B$ is the surface of the body. In case of a force-free body, the surface term vanishes.

[107] D. V. Anghel and T. Kühn, Quantization of the Elastic Modes in an Isotropic Plate, J. Phys. A 40, 10429 (2007).

[108] A. E. Armenàkas, D. C. Gazis, and G. Herrmann, Free Vibrations of Circular Cylindrical Shells (Pergamon Press, Oxford, 1969). 
[109] T. R. Meeker and A. H. Meitzler, Guided Wave Propagation in Elongated Cylinders and Plates, in Methods and Devices, Part A, Physical Acoustics: Principles and Methods, Vol. I A, edited by W. P. Mason (Academic Press, New York, 1964), pp. 111-167.

[110] A. C. Eringen and E. S. Şuhubi, Elastodynamics: Linear Theory (Academic Press, New York, 1975), Vol. 2.

[111] Equation (C26) is only valid if all subdeterminants of $\boldsymbol{M}$ are nonzero, as is usually the case for flexural modes. Otherwise, the flexural family decomposes into different independent mode families, with frequency equations determined by the respective subdeterminants.

[112] C. Holmes, Direct UV Written Planar Devices for Sensing and Telecommunication Applications, Ph.D. thesis, University of Southampton, Optoelectronics Research Centre, 2009.

[113] J. J. Sakurai and J. Napolitano, Modern Quantum Mechanics (Addison-Wesley, San Francisco, 2011).
[114] The chain rule states that the derivative of a composition of functionals $(F \circ G)[\boldsymbol{x}] \equiv F(G[\boldsymbol{x}])$ evaluated at $\boldsymbol{x}=\boldsymbol{a}$ and in direction $\boldsymbol{n}$ is the derivative of the outer functional $F$ in the direction of the derivative of the inner functional $G$ [64]

$$
D(F \circ G)_{\boldsymbol{a}}[\boldsymbol{n}]=D F_{G[\boldsymbol{A}]}\left(D G_{\boldsymbol{a}}[\boldsymbol{n}]\right)
$$

analogous to the chain rule for ordinary functions.

[115] Handbook of Optics: Devices, Measurements, and Properties, 2nd ed., edited by M. Bass, E. W. Van Stryland, D. R. Williams, and W. L. Wolfe (McGraw-Hill, New York, 2001), Vol. 2.

[116] K. Vedam, The Elastic and Photoelastic Constants of Fused Quartz, Phys. Rev. 78, 472 (1950).

[117] J. Meija, T. B. Coplen, M. Berglund, W. A. Brand, P. De Bièvre, M. Gröning, N. E. Holden, J. Irrgeher, R. D. Loss, T. Walczyk, and T. Prohaska, Atomic Weights of the Elements 2013 (IUPAC Technical Report), Pure Appl. Chem. 88, 265 (2016). 